TECHNICAL REPORT STANDARD TITLE PAGE

\begin{tabular}{|c|c|c|c|c|}
\hline $\begin{array}{l}\text { 1. Report No. } \\
\text { FHWA/IN/JTRP-2002/29 }\end{array}$ & \multicolumn{2}{|c|}{ 2. Government Accession No. } & \multicolumn{2}{|c|}{ 3. Recipient's Catalog No. } \\
\hline \multirow{2}{*}{\multicolumn{3}{|c|}{$\begin{array}{l}\text { 4. Title and Subtitle } \\
\text { Asphalt Additives to Control Rutting and Cracking }\end{array}$}} & \multicolumn{2}{|c|}{$\begin{array}{l}\text { 5. } \quad \text { Report Date } \\
\text { January } 2003\end{array}$} \\
\hline & & & \multicolumn{2}{|c|}{ 6. Performing Organization Code } \\
\hline \multicolumn{3}{|c|}{$\begin{array}{l}\text { 7. Author(s) } \\
\text { Rebecca McDaniel and Ayesha Shah }\end{array}$} & \multicolumn{2}{|c|}{$\begin{array}{l}\text { 8. Performing Organization Report No. } \\
\text { FHWA/IN/JTRP-2002/29 }\end{array}$} \\
\hline \multirow{2}{*}{\multicolumn{3}{|c|}{$\begin{array}{l}\text { 9. Performing Organization Name and Address } \\
\text { Joint Transportation Research Program } \\
1284 \text { Civil Engineering Building } \\
\text { Purdue University } \\
\text { West Lafayette, IN } 47907-1284\end{array}$}} & \multicolumn{2}{|c|}{ 10. Work Unit No. } \\
\hline & & & \multicolumn{2}{|c|}{$\begin{array}{l}\text { 11. Contract or Grant No. } \\
\text { DTFH } 7188-5039\end{array}$} \\
\hline \multirow{2}{*}{\multicolumn{2}{|c|}{$\begin{array}{l}\text { 12. Sponsoring Agency Name and Address } \\
\text { Federal Highway Administration } \\
575 \text { N. Pennsylvania, Room } 254 \\
\text { Indianapolis, IN } 46204\end{array}$}} & & \multicolumn{2}{|c|}{$\begin{array}{l}\text { 13. Type of Report and Period Covered } \\
\text { Final Report }\end{array}$} \\
\hline & & & \multicolumn{2}{|c|}{ 14. Sponsoring Agency Code } \\
\hline \multicolumn{5}{|c|}{$\begin{array}{l}\text { 15. Supplementary Notes } \\
\text { Prepared in cooperation with the Federal Highway Administration and the Indiana Department of Transportation. }\end{array}$} \\
\hline \multicolumn{5}{|c|}{$\begin{array}{l}\text { 16. Abstract } \\
\text { This report presents the results of an investigation of the performance of a variety of materials added to asphalt binders and } \\
\text { mixtures to change their properties, particularly with respect to rutting and cracking. The approach included a field trial of } \\
\text { seven polymer and particulate modifiers, supplemented by laboratory characterization of the materials used in the field. The } \\
\text { modifiers evaluated included PAC, Novophalt, Multigrade asphalt cement, polyester fibers, Neoprene, SBR and asphalt } \\
\text { rubber. The field trial showed that different modifiers do yield different performance. Modifiers are not essential to ensure } \\
\text { that the pavement will not rut. None of the mixtures evaluated here exhibited appreciable rutting. Dramatic differences } \\
\text { were noted in the cracking behavior, however. Newly developed laboratory tests were able to identify binders that would be } \\
\text { more prone to cracking. All of the materials evaluated did change the properties of the binders or mixtures in some way. } \\
\text { Some of the modifiers, however, were more effective at modifying the properties to provide improved field performance in a } \\
\text { cost effective manner. }\end{array}$} \\
\hline \multicolumn{2}{|c|}{$\begin{array}{l}\text { 17. Key Words } \\
\text { Superpave, hot mix asphalt, rutting, cracking, modifiers, } \\
\text { modified binders, binder testing. }\end{array}$} & \multicolumn{3}{|c|}{$\begin{array}{l}\text { 18. Distribution Statement } \\
\text { No restrictions. This document is available to the public through the } \\
\text { National Technical Information Service, Springfield, VA } 22161\end{array}$} \\
\hline 19. Security Classif. (of this report) & 20. Security Classif. & is page) & 21. No. of Pages & 22. Price \\
\hline Unclassified & Unclas & & 120 & \\
\hline
\end{tabular}

Form DOT F 1700.7 (8-69) 


\section{TECHNICAL Summary}

INDOT Research

Technology Transfer and Project Implementation Information

TRB Subject Code:31-2 Bituminous Materials

Publication No.: FHWA/IN/JTRP-2002/29 DTFH-7188-5039

January 2003

Final Report

\section{Asphalt Additives to Control Cracking and Rutting}

\section{Introduction}

Prior to the implementation of the Superpave asphalt binder specifications, various asphalt modifiers were being promoted to improve the performance of hot mix asphalt (HMA) pavements. Specifying agencies faced many difficult decisions when they chose to specify modifiers. For example, which modifier was best for a given set of circumstances? Modified binders also tended to be significantly more expensive than neat, or unmodified, asphalts. Material costs alone could be 25 to $100 \%$ higher than conventional asphalts. Construction costs could also be increased due to the need for higher temperatures, additional equipment and other special handling requirements. These additional costs made it harder to justify the use of modified binders without reliable performance histories.

The implementation of the Superpave performance graded binder specifications introduced a new way to specify an asphalt binder for a particular hot mix asphalt application and was viewed by many as a solution to the problems of dealing with modified binders. Under Superpave, a binder is selected to provide sufficient stiffness to resist rutting at expected high service temperatures and enough flexibility to resist fatigue and thermal cracking at intermediate and low service temperatures. The wider the range of temperatures at which a binder must perform, the more difficult it is to span the range with an unmodified binder. The original purpose of this field trial and laboratory study was to evaluate the performance of seven different asphalt modifiers, representing some of the major types of modifiers, to determine which provided the best, most cost-effective performance improvement in terms of rutting and cracking resistance. All seven modifiers were included in hot mix asphalt mixtures placed on one project on I-465 in 1990. Two control sections using AC-20 were also constructed on the same project.

During the course of this study, the Indiana Department of Transportation (INDOT) adopted the Superpave binder specifications and subsequently the Superpave mix design process. This study then offered an excellent opportunity to evaluate the modified binders used on this project according to the Superpave binder tests and to relate those test results to actual, long-term service performance. It also offered a unique opportunity to evaluate proposed new protocols for modified binder testing under AASHTO MP1a.

\section{Findings}

After 11 years of service, the field sections were all performing well in terms of rutting. No appreciable rutting had been measured on any of the sections. The fact that no rutting occurred on this project can be attributed to INDOT's revised Marshall mix design practices, coupled with high quality aggregates and close attention to detail during construction by the agency and contractor.

There are marked differences, however, between the various sections in terms of cracking.
Some of the sections cracked extensively within three to six years after construction. Other sections were still performing well after 11 years. It should be noted, however, that the binders did not all meet the same performance grade, so differences could be expected. The best performers included the SBR, PAC and AR. A second tier of performance included the Neoprene, Fibers and MGAC. The worst 
performers were the unmodified control sections and the Novophalt.

The observed cracking was not merely reflective cracking from the underlying portland cement concrete layer as it did not all correspond to underlying cracks or joints. In addition, the worst performers exhibited extensive longitudinal cracking, which could not be caused by the underlying concrete. Much of the cracking was apparently caused by brittleness of the binder, especially in the Novophalt section. No other significant distresses were noted during field surveys.

The proposed new (MP1a) binder test results identified the polyethylene modifier as the most prone to cracking. The control binder (AC-20) was also identified as likely to crack. This lends credence to the recommended new procedures for testing modified binders. Conventional PG testing, however, also ranked the binders correctly in terms of cracking. With this limited testing, the PG tests appeared to be as accurate as the MP1a testing, which is much more time consuming. At this point and based on this limited data, there does not appear to be a compelling need to implement MP1a. That situation may change in the future as more data is collected and analyzed.

High temperature binder testing revealed that all of the modifiers stiffened the binder. High temperature binder stiffness relates to pavement rutting. As no rutting was observed in the field, no significant laboratory differences were expected. The aggregate framework and overall mix design have a greater influence on rutting than the binder.

Indirect tensile testing performed on selected samples of the modified and control mixtures did not correlate with the observed field performance.

\section{Implementation}

The results of this project show that modification of the binder is not necessary to produce pavements that will not rut under heavy traffic. Even the control sections performed well here with only minimal rutting. Good aggregate gradations, proper mix designs and close attention to detail during construction contributed to the good rutting performance. INDOT's approach of using Superpave mix designs, quality control/quality assurance specifications and some warranty specifications as well, help to ensure that good mixes are designed and constructed.

This study also shows that modifiers can improve the cracking resistance of the mixtures in which they are used, provided the modified binders meet the PG grade appropriate for the location. The materials that performed poorly in terms of cracking only met a low temperature grade of -16 . Those that met a -22 or -28 grade performed much better.

It appears, based on this limited data from one site with only eight binders, that the conventional PG low temperature tests (MP1) using the BBR were able to rank the binders in terms of cracking resistance as well as the newly proposed tests under MP1a. Thus, while the MP1a testing may be preferred from a theoretical point of view, in this particular case, it does not appear to offer a great improvement in the ability to predict cracking compared to the simpler MP1 testing. Additional information is needed to ascertain whether INDOT should move to adopt MP1a, though based on this data, there does not seem to be a compelling reason to do so. Additional efforts are underway on a national level to verify the need to implement MP1a testing.

\section{Contacts}

For more information:

Dr. Rebecca S. McDaniel

Principal Investigator

Technical Director

North Central Superpave Center

Purdue University

West Lafayette IN 47907

Phone: (765) 463-2317

Fax: (765) 497-2402

\section{Indiana Department of Transportation}

Division of Research

1205 Montgomery Street

P.O. Box 2279

West Lafayette, IN 47906

Phone: (765) 463-1521

Fax: (765) 497-1665

Purdue University

Joint Transportation Research Program

School of Civil Engineering

West Lafayette, IN 47907-1284

Phone: (765) 494-9310

Fax: (765) 496-1105 
Final Report

FHWA/IN/JTRP-2002/29

\title{
ASPHALT ADDITIVES TO CONTROL RUTTING AND CRACKING
}

\author{
Conducted by \\ Rebecca S. McDaniel, Technical Director \\ Ayesha Shah, Research Engineer \\ North Central Superpave Center \\ P. O. Box 2382 \\ 1205 Montgomery Street \\ West Lafayette, IN 47906 \\ Joint Transportation Research Program \\ Project No. C-36-56M \\ File No. 2-13-13 \\ DTFH 7188-5039
}

The contents of this report reflect the views of the authors who are responsible for the facts and the accuracy of the data presented herein. The contents do not necessarily reflect the official views or policies of the Indiana Department of Transportation or the Federal Highway Administration. This report does not constitute a standard, specification or regulation.

Purdue University

West Lafayette, IN 47907

January 2003 


\section{TABLE OF CONTENTS}

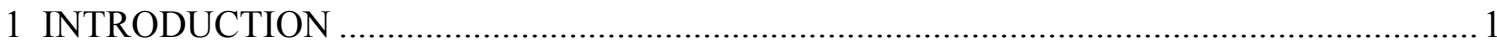

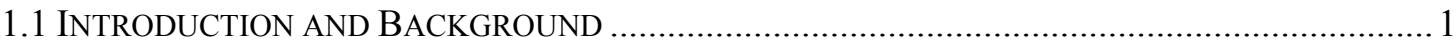

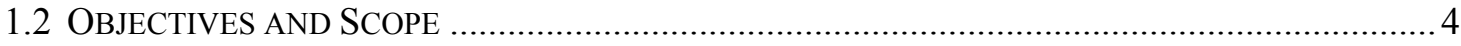

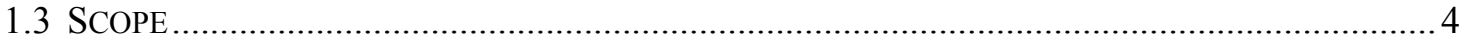

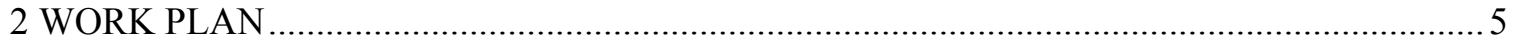

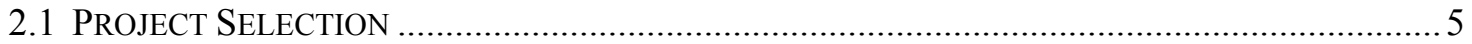

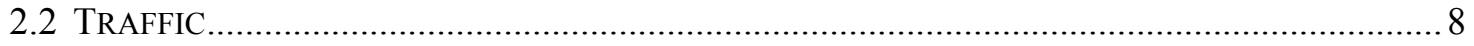

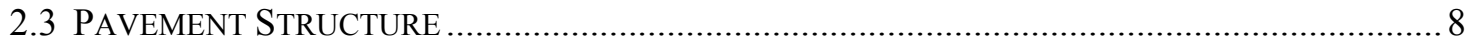

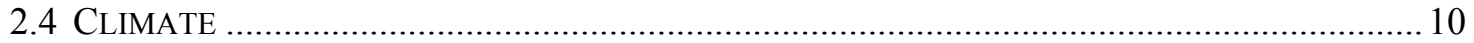

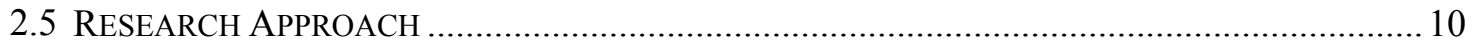

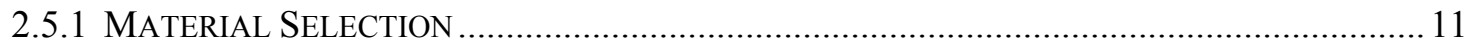

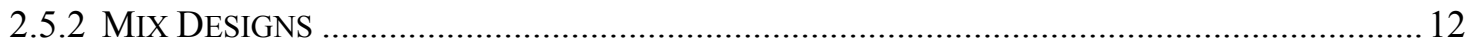

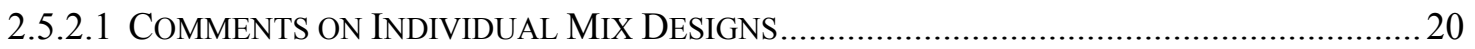

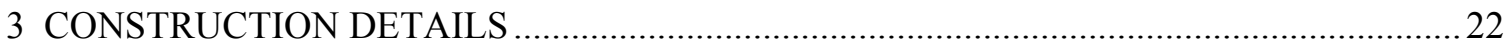

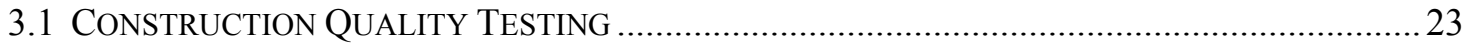

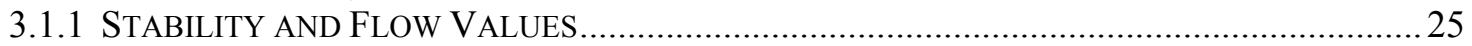

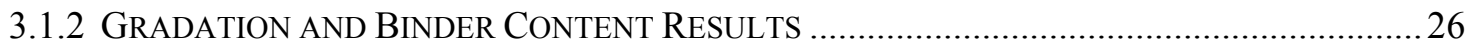

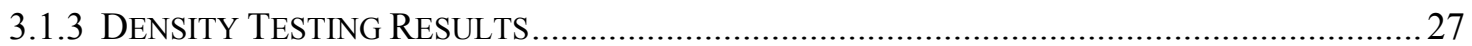

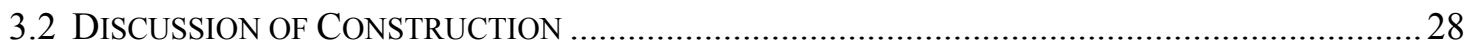

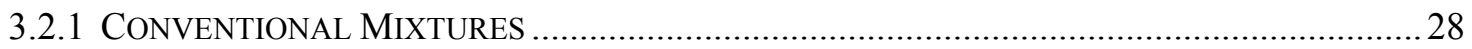

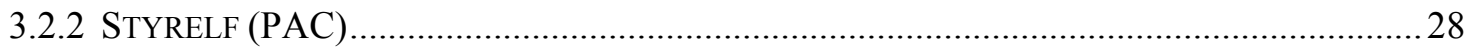

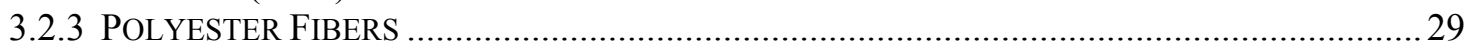

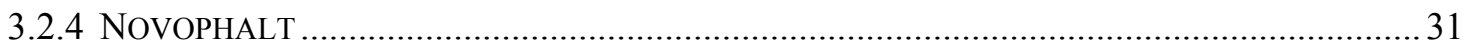

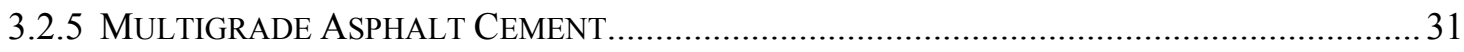

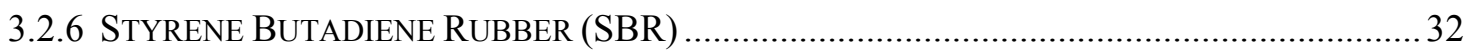

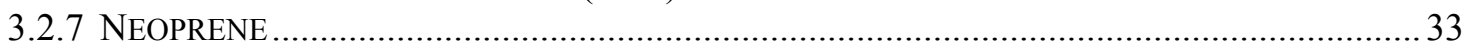

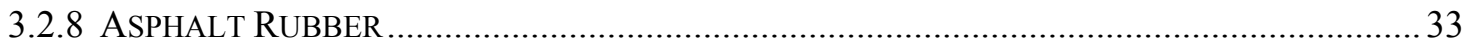

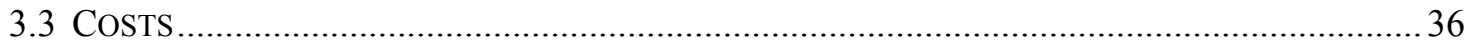

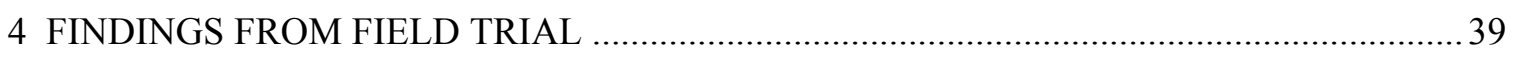

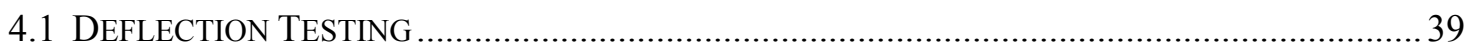

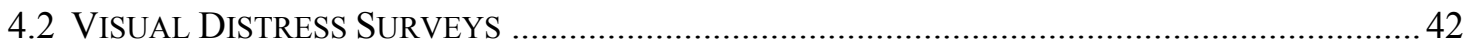

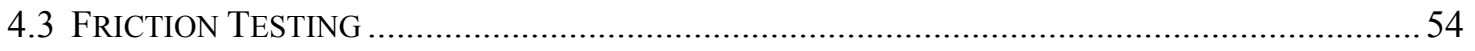

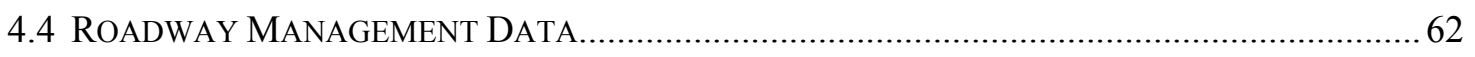

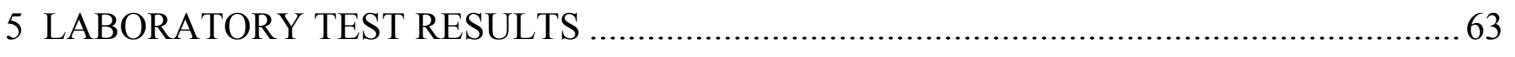

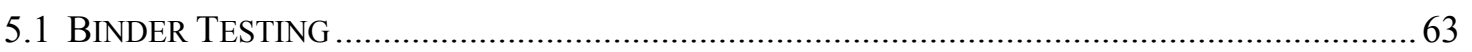

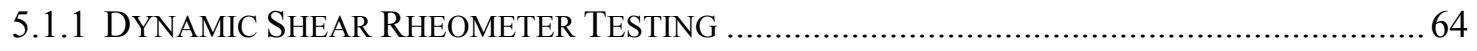

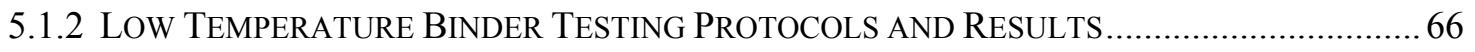

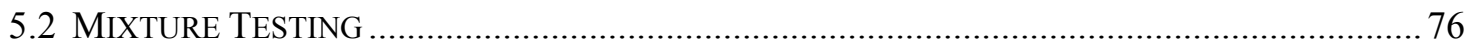

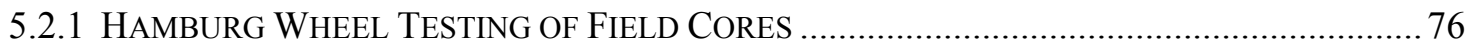

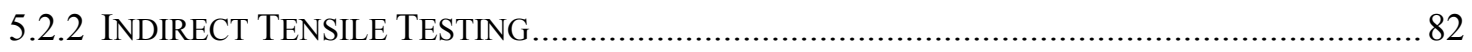




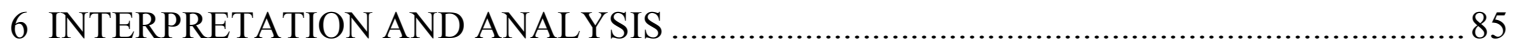

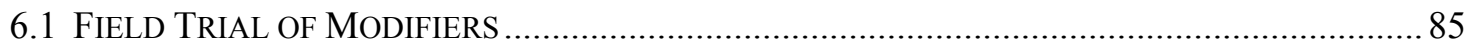

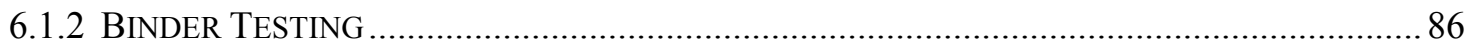

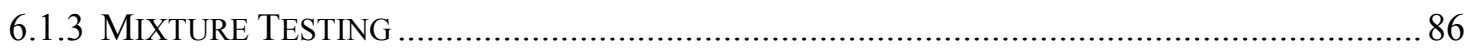

6.1.4 RELATIONSHIP OF LABORATORY RESULTS TO FIELD PERFORMANCE ............................ 87

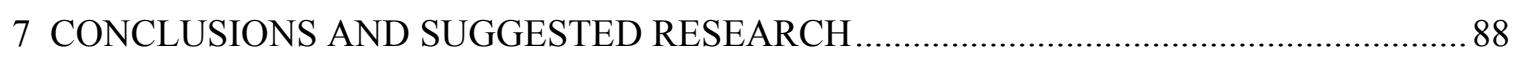

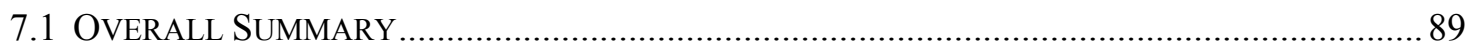

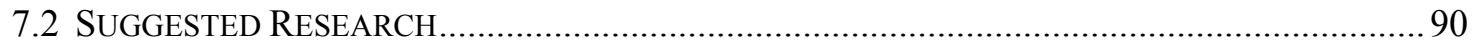

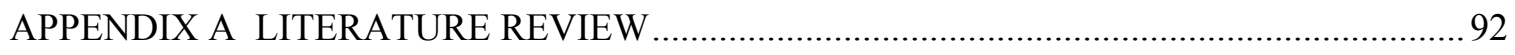

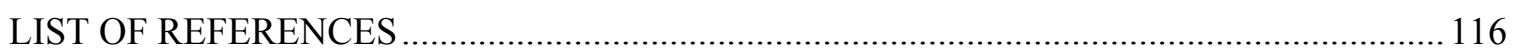




\section{LIST OF TABLES}

Table Page

Table 1 Generic Classification of Asphalt Modifiers (6)............................................. 3

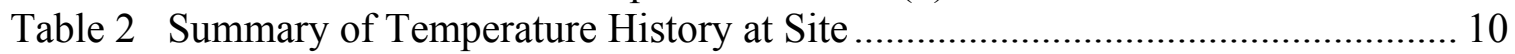

Table 3 Modifiers Used in the Study ....................................................................... 11

Table 4 No. 5 Base Course Mix Design Parameters.................................................... 13

Table 5 No. 9 Intermediate (Binder) Course Mix Design Parameters............................ 14

Table 6 No. 11 Surface Course Mix Design Parameters ............................................. 15

Table 7 No. 5 Base Course Mix Design Gradations................................................... 16

Table 8 No. 9 Intermediate (Binder) Course Mix Design Gradations ........................... 17

Table 9 No. 11 Surface Course Mix Design Gradations ................................................ 18

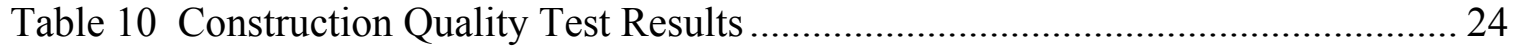

Table 11 Test Strip Core Density .................................................................................... 27

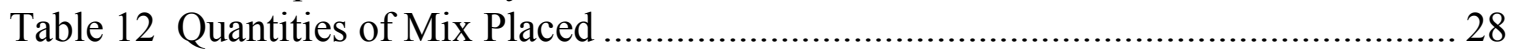

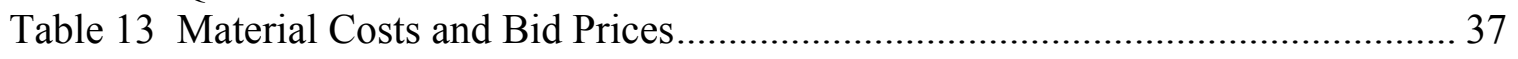

Table 14 Summary of Dynaflect Parameters ............................................................... 41

Table 15 I-465 Pavement Condition Surveys - Cracking Summary ............................. 42

Table 16 I-465 Condition Surveys - Rutting Summary …......................................... 52

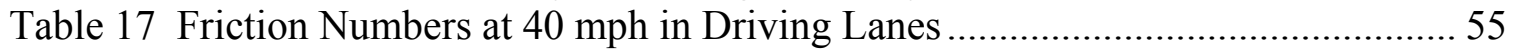

Table 18 Friction Numbers at $40 \mathrm{mph}$ in Center Lanes.................................................. 56

Table 19 Friction Numbers at $40 \mathrm{mph}$ in High Speed Lanes........................................ 56

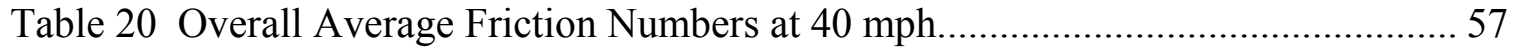

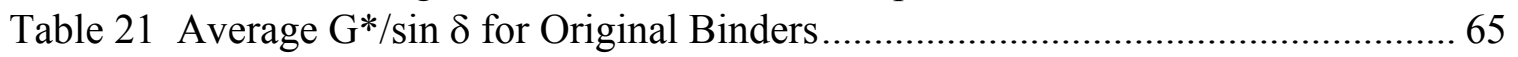

Table 22 Low Temperature Binder Testing Under Revised Protocols........................... 69

Table 23 Estimates of Critical Cracking Temperature for Modified Binders ................ 72

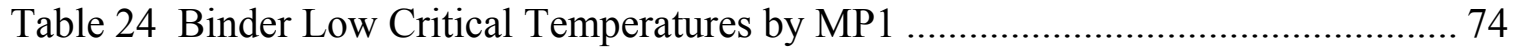

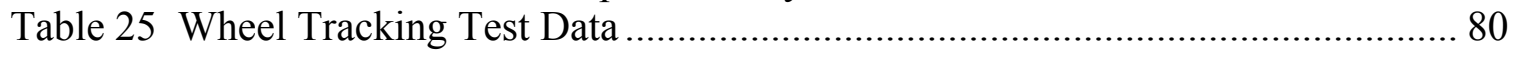

Table 26 Summary of Wheel Tracking Performance Rankings ................................. 81

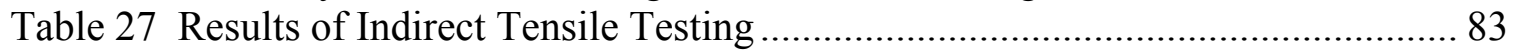




\section{LIST OF FIGURES}

Figure

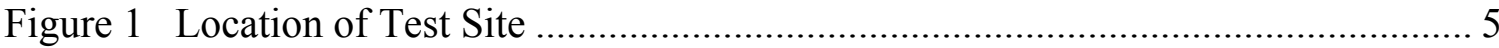

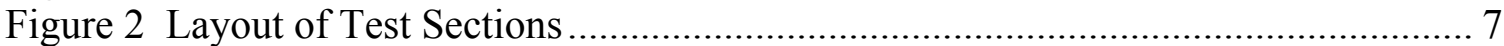

Figure 3 Pavement Cross Section .............................................................................. 9

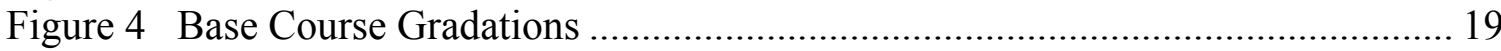

Figure 5 Intermediate Course Gradations ................................................................ 19

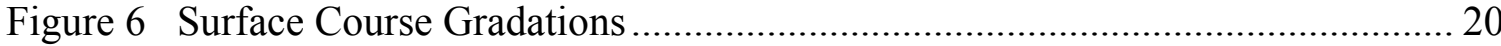

Figure 7 Hot Mix Batch Plant Used on Project ......................................................... 23

Figure 8 Neoprene, MGAC and PAC Were Metered into AC Line at Plant.................. 29

Figure 9 Fibers in Bags on Conveyor to Weigh Hopper .............................................. 30

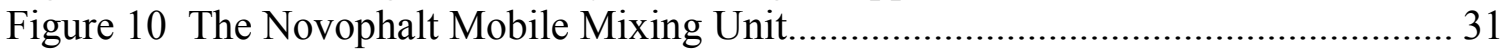

Figure 11 Pumping SBR Latex from Barrel into Plant................................................... 32

Figure 12 Meter Used to Control Flow of SBR into Plant .............................................. 33

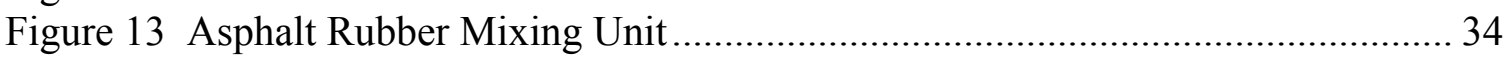

Figure 14 Ground Tire Rubber Used in Asphalt Rubber Binder ................................... 34

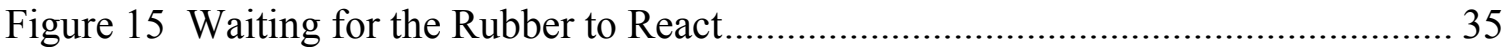

Figure 16 Pumping Reacted Asphalt Rubber into Plant ................................................. 36

Figure 17 Development of Cracking in Control Section (A).......................................... 44

Figure 18 Development of Cracking in PAC Section (B) .................................................... 45

Figure 19 Development of Cracking in Fiber Section (C)............................................ 45

Figure 20 Development of Cracking in PE Section (D) .............................................. 46

Figure 21 Development of Cracking in MGAC Section (E) ………………………..... 47

Figure 22 Development of Cracking in SBR Section (F) ............................................ 48

Figure 23 Development of Cracking in Neo Section $(\mathrm{G})$.............................................. 49

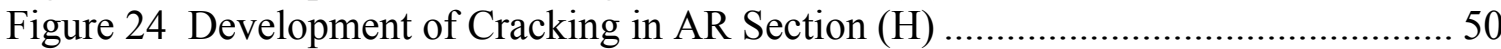

Figure 25 Development of Cracking in Control Section (J) ............................................ 51

Figure 26 Average Friction Number vs. Time in Driving Lane ..................................... 57

Figure 27 Average Friction Number vs. Time in Center Lane .......................................58

Figure 28 Average Friction Number vs. Time in High Speed Lane................................. 58

Figure 29 Overall Average Friction Number vs. Time (All Lanes) ................................. 59

Figure 31 Effect of Modification on Failure Strain Properties ........................................ 71

Figure 32 Critical Cracking Temperatures .............................................................. 72

Figure 33 Correlations of Cracking Counts Measured in 2001 with Direct Tension

Results and Critical Cracking Temperatures ............................................................ 73

Figure 34 Critical Temperature by S Value vs. Cracking.............................................. 75

Figure 35 Critical Temperature by m-value vs. Cracking ………………………….... 75

Figure 36 Generalized Wheel Tracking Parameters (from 60)...................................... 77

Figure 37 Wheel Tracking Results on Modified Mixes................................................. 78

Figure 38 Estimated Thermal Stress and Strength of the PAC Surface ………….......... 84 


\section{ACKNOWLEDGEMENTS}

Countless people have helped and encouraged me through this long project. Some to whom I am particularly grateful include: the people from Reith-Riley who built the project, particularly Dudley Bonte, Quentin Stout and Rusty Mann; the Greenfield Construction and Testing personnel who oversaw the project, including Rob Goldner, Fred Williams and Dale Eastin; all of the material suppliers who participated wholeheartedly in the project; Larry Bateman, Idris Jones, Carl Berryman and Sedat Gulen for the friction data; John Weaver, Bill Flora, Dave Holtz and Eric Conklin for assistance in getting the Roadway Management data; Arthur Rucker, Ron Walker and Clyde Lovelady for help in setting up the project and getting project testing results; Calvin Reck, Travis Lovvorn, Shakor Badaruddin, Khaled Galal, Steve Bowman and Kevin Brower for coring and counting cracks; and Dr. Hussain Bahia and Kitae Nam at the University of Wisconsin for the binder testing under the NCHRP 9-10 protocols; James Esler for the DSR testing. Thanks to Lynn Warble for turning my field notes into beautiful crack maps. 


\section{INTRODUCTION}

\subsection{Introduction and Background}

Prior to the implementation of the Superpave asphalt binder specifications, various asphalt modifiers were being promoted to improve the performance of hot mix asphalt (HMA) pavements. Specifying agencies faced many difficult decisions when they chose to specify modifiers. For example, which modifier was best for a given set of circumstances? There was little information available to guide the selection of the appropriate modifier for a given situation. In addition, many modifiers were proprietary products, so specifying one particular modifier removed or reduced competition significantly. (The use of proprietary products is usually avoided in low-bid contracts.) Attempts were made to write broad, generic specifications for modified asphalts, but these were not widely adopted.

Modified binders also tended to be significantly more expensive than neat, or unmodified, asphalts. Material costs alone could be 25 to $100 \%$ higher than conventional asphalts. Construction costs could also be increased due to the need for higher mixing and compaction temperatures, additional equipment for adding or handling the modifier, and other special handling requirements. These additional costs made it harder to justify the use of modified binders without reliable performance histories.

Although the use of asphalt modifiers did offer the potential for improved performance, then, many states found it difficult or impossible to specify their use. Occasional usage could be justified as a research effort. Those states that did adopt the use of modifiers tended to find one class of modifiers that had demonstrated good performance under their specific local conditions, and then they specified that type of modifier exclusively.

The implementation of the Superpave performance graded binder specifications introduced a new way to test and select an asphalt binder for a particular hot mix asphalt application. A binder is selected to provide sufficient stiffness to resist rutting at expected high service temperatures and enough flexibility to resist fatigue and thermal cracking at intermediate and low service temperatures.

The wider the range of temperatures at which a binder must perform, the more difficult it is to span the range with an unmodified binder. A rough rule of thumb states that when the range of temperatures is greater than about $90^{\circ} \mathrm{C}$, the likelihood increases that some form of 
modification will be necessary. A wide variety of materials may be used to modify the behavior or properties of asphalt. Table 1 shows some of the typical categories of modifiers, according to a classification scheme devised by Terrel and Epps (므). More information on types of modifiers is provided in the literature review in Appendix A.

The Superpave system was viewed by many as a solution to the problems of dealing with modified binders. In original concept, the Superpave binder system was intended to be "blind" to the use of modifiers. (무) The specifications required certain levels of performance at high, intermediate and low temperatures. Test temperatures were related to the actual temperatures that would be encountered during production and in-service. The suppliers would modify as necessary to attain the required properties. The buyer could be unaware of the details of the modification.

As the Superpave binder specification was implemented, however, it became clear that the situation was not as simple as originally envisioned. Some modifiers interfered with the tests or violated the basic assumptions underlying the tests. For example, some particulate materials were too large and caused problems in the sample geometry of the dynamic shear rheometer tests, where a 1 to $2 \mathrm{~mm}$ thick sample is tested. Other modifiers displayed non-Newtonian behavior and therefore violated one of the assumptions of the test. (4) From a practical standpoint, many modified binders were "sticky" and hard to fabricate into the specific specimen geometries needed.

The National Cooperative Highway Research Program initiated a study (Project 9-10) to investigate the applicability of the Superpave binder tests to modified binders. This research was designed to recommend changes to the binder testing protocols for modified binders and to identify problems with characterizing modified mixes using the Superpave mixture performance tests. After extensive study, the researchers recommended a number of changes to the binder testing protocols to account for the fact that many modified systems violate the simplifying assumptions of the Performance Grading specification. Recommendations were made to improve the effectiveness of Superpave mixture testing procedures as well. (무) 
Table 1 Generic Classification of Asphalt Modifiers (ㅁ)

\begin{tabular}{|c|c|}
\hline TYPE OF MODIFIER & Examples \\
\hline 1. Filler & $\begin{array}{l}\text { - } \text { Mineral Filler: crusher fines, lime, portland cement, fly ash } \\
\text { - } \quad \text { Carbon black } \\
\text { - Sulfur }\end{array}$ \\
\hline 2. Extender & $\begin{array}{ll} & \text { Sulfur } \\
\text { - } & \text { Lignin }\end{array}$ \\
\hline $\begin{array}{l}\text { 3. Rubber } \\
\text { a. Natural latex } \\
\text { b. Synthetic latex } \\
\text { c. Block copolymer } \\
\text { d. Reclaimed rubber }\end{array}$ & $\begin{array}{ll}\text { - } & \text { Natural rubber } \\
\text { - } & \text { Styrene-butadiene or SBR } \\
\text { - } & \text { Styrene-butadiene-styrene or SBS } \\
\text { - } & \text { Recycled tires }\end{array}$ \\
\hline 4. Plastic & $\begin{array}{ll}\text { - } & \text { Polyethylene } \\
\text { - } & \text { Polypropylene } \\
\text { - } & \text { Ethyl-vinyl-acetate, EVA } \\
\text { - } & \text { Polyvinyl chloride, PVC }\end{array}$ \\
\hline 5. Combination & - $\quad$ Blends of polymers in 3 and 4 \\
\hline 6. Fiber & $\begin{array}{ll}\text { - } & \text { Natural: Asbestos, rock wool } \\
\text { - } & \text { Man-made: Polypropylene, polyester, fiberglass }\end{array}$ \\
\hline 7. Oxidant & - $\quad$ Manganese salts \\
\hline 8. Antioxidant & $\begin{array}{l}\text { - Lead compounds } \\
\text { - Carbon } \\
\text { - Calcium salts }\end{array}$ \\
\hline 9. Hydrocarbon & $\begin{array}{l}\text { - } \text { Recycling and rejuvenating oils } \\
\text { - Hardening and natural asphalts }\end{array}$ \\
\hline 10. Antistrip & $\begin{array}{l}\text { - Amines } \\
\text { - } \quad \text { Lime }\end{array}$ \\
\hline
\end{tabular}




\subsection{Objectives and Scope}

The original purpose of this field trial was to evaluate the performance of seven different asphalt modifiers, representing some of the major types of modifiers, to determine which provided the best, most cost-effective performance improvement. The primary concern was to identify additives that could improve the rutting resistance of hot mix asphalt overlays, as premature rutting was a significant problem at the time this study was initiated. (The study was originally conceived in 1987, but test sections were not constructed until 1990.) One concern at the time was that rutting might be improved by stiffening the binder to the point that the mixture became more susceptible to cracking. Therefore, this study was also designed to investigate the binders' effects on cracking. Additives were sought that could improve both rutting and cracking resistance.

During the course of this study, the Indiana Department of Transportation (INDOT) adopted the Superpave binder specifications and subsequently the Superpave mix design process. INDOT saw the Superpave binder specifications as a major improvement over the viscosity grading system it had been using, as well as a way to avoid the issue of specifying particular, proprietary modifiers. This study offered an excellent opportunity to evaluate the modified binders used on this project according to the Superpave binder tests and to relate those test results to actual, long-term service performance.

\subsection{Scope}

This project is a laboratory and field investigation of the performance of seven asphalt additives to control rutting and cracking. All seven modifiers were included in hot mix asphalt mixtures placed on one project on I-465 in 1990. Two control sections using AC-20 were also constructed on the same project. 


\section{WORK PLAN}

This chapter outlines the field project, materials tested and test protocols followed for this research effort.

\subsection{Project Selection}

The highway selected for construction of the test sections used in this research was I-465, the ring road around Indianapolis. The project is located on the west side of town along the east side of the Indianapolis International Airport at approximately $39^{\circ} 44^{\prime}$ North latitude and $86^{\circ} 16^{\prime}$ West longitude. The elevation of the site is approximately $241 \mathrm{~m} \mathrm{(791} \mathrm{ft.)} \mathrm{above} \mathrm{mean} \mathrm{sea} \mathrm{level.}$ The specific project location begins $0.6 \mathrm{~km}$ ( 0.4 miles) north of US-40 and continues to State Road 67 for a total length of $5.0 \mathrm{~km}$ (3.3 miles). Test sections are located in both the north and southbound directions; a control section with conventional AC-20 is included in each direction. The project location is shown in Figure 1. Figure 2 shows the layout of the individual test sections.

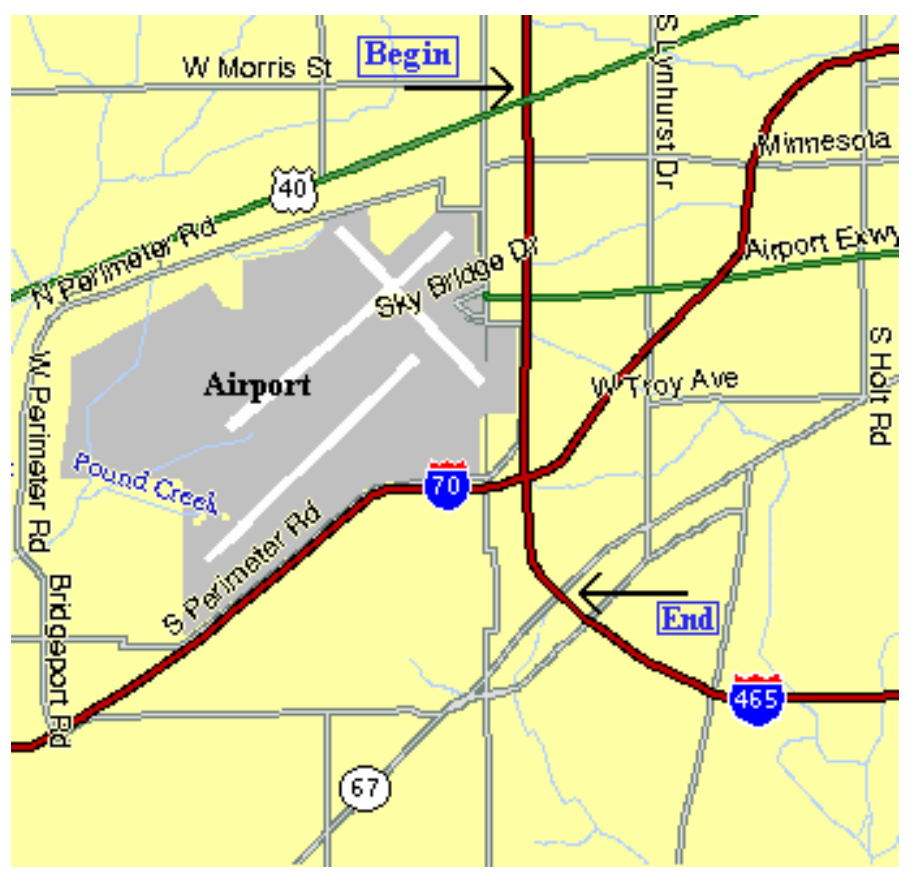

Figure 1 Location of Test Site 
This site was selected based on input from the INDOT Divisions of Design, Materials and Tests, and Research. In order to constitute a fair test of the modifiers' performance and to be able to differentiate between the modifiers, a site with a high traffic volume was sought. The requirements for suitable test sites included:

- Resurfacing or overlay project,

- Sufficient length to allow test sections of at least one-half mile in the travel lane plus a control section of equal length,

- Uniform traffic volume throughout the length of the project, and

- Comparable pavement structures throughout.

The chosen section on I-465 met all of these requirements, for the most part. Due to the presence of ramps within the project length, the traffic volume is not completely uniform. 


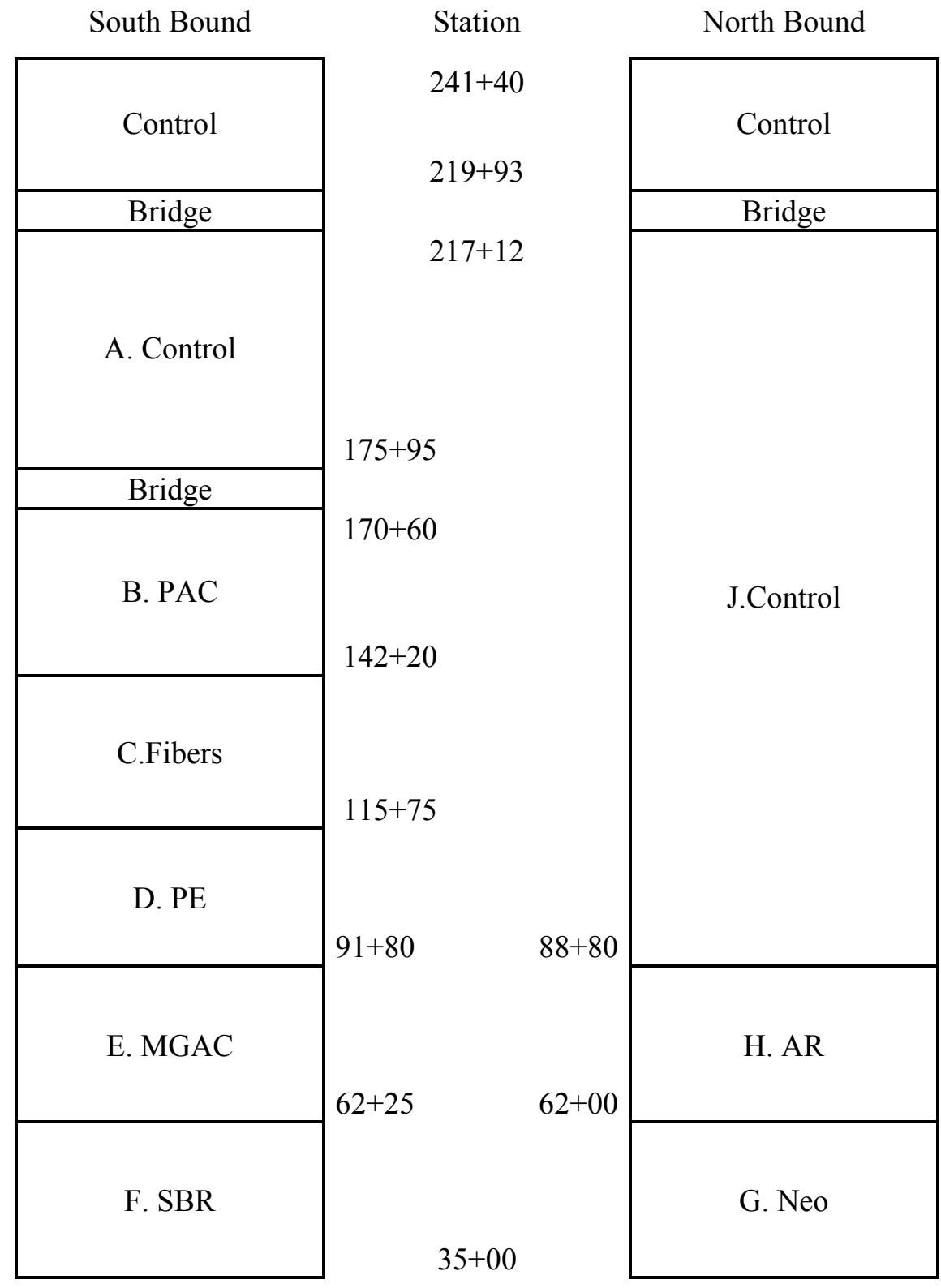

Total Length $=5.0 \mathrm{~km}(3.3 \mathrm{~km})$

Figure 2 Layout of Test Sections 


\subsection{Traffic}

Weigh in motion (WIM) data from INDOT collected in 1999 shows that the traffic volume through the test sections was approximately 150,000 vehicles per day with about $30 \%$ trucks. The traffic volume no doubt changes somewhat through the total project length as traffic enters and leaves I-465 at ramps to the Indianapolis International Airport and to I-70. Specific details on the changes in traffic volume were not readily available without special WIM counts.

Since traffic related distresses, such as rutting, were minimal and since the pavement performance did not appear to vary by location between the ramps, no special WIM counts were requested.

\subsection{Pavement Structure}

Figure 3 illustrates the pavement cross section used on this project. This represents the standard overlay design in use in Indiana at the time of construction. While the Dynaflect was used in the 1980's and 1990's to determine where concrete pavements needed to be undersealed to improve the support conditions, no rigorous structural design procedure was in use at that time.

A variable base course thickness was used to change the crown of the roadway. This was routine in Indiana when overlaying concrete pavements. The cross section of all of the test sections is consistent except for the end of section F, the SBR section, and the first portion of Section $\mathrm{G}$, the Neoprene section. In this area, the underlying pavement was superelevated at a curve. This area was avoided during condition surveys to the extent possible. As a general observation, however, no particular performance differences were noted in this area as compared to other parts of the same sections. 


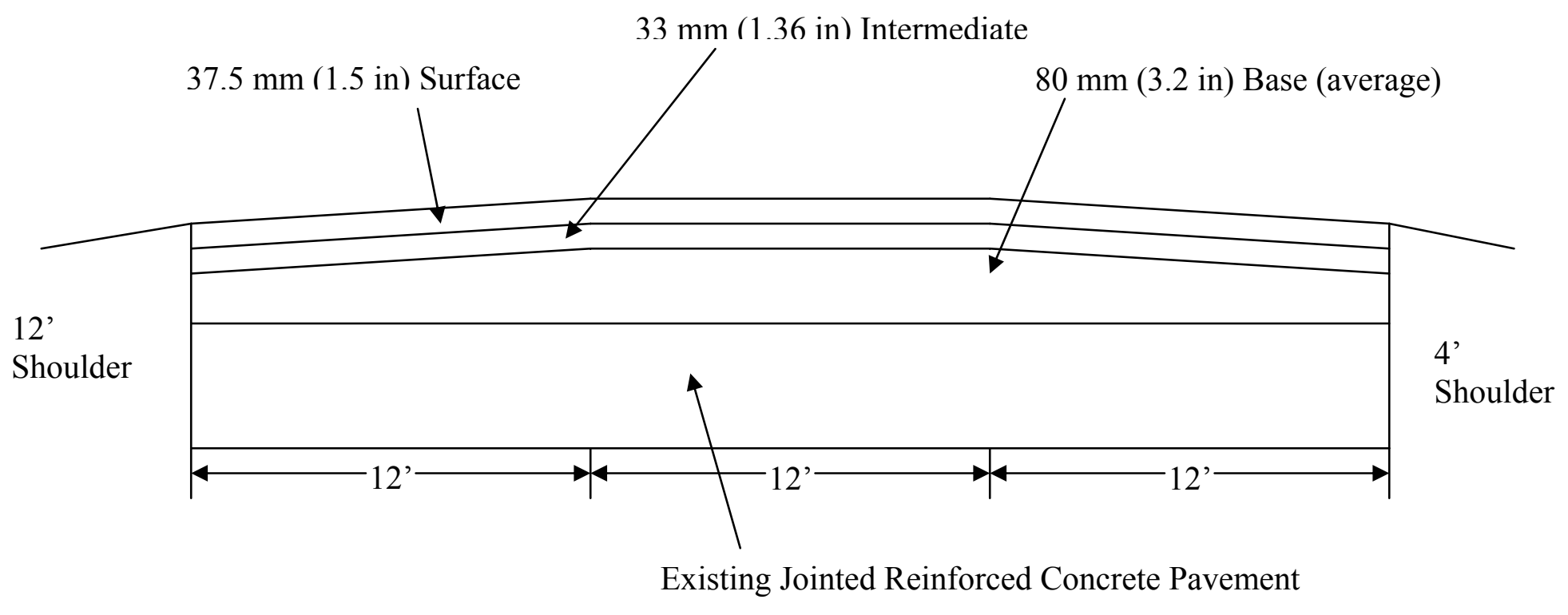

Figure 3 Pavement Cross Section 


\subsection{Climate}

The proximity of the project to the Indianapolis International Airport provides an excellent climatological record. The LTPPBind program (64) summarizes that record over the last 48 years (prior to 1996). LTPPBind includes the following summary of the weather history of the site.

Table 2 Summary of Temperature History at Site

\begin{tabular}{|l|c|c|c|c|c|}
\hline \multicolumn{1}{|c|}{ Temperature } & $\begin{array}{c}\text { Mean, } \\
{ }^{\circ} \mathrm{C}\end{array}$ & $\begin{array}{c}\text { Standard } \\
\text { Deviation, }{ }^{\circ} \mathrm{C}\end{array}$ & $\begin{array}{c}\text { Minimum, } \\
{ }^{\circ} \mathrm{C}\end{array}$ & $\begin{array}{c}\text { Maximum, } \\
{ }^{\circ} \mathrm{C}\end{array}$ & $\begin{array}{c}\text { Years in } \\
\text { Record }\end{array}$ \\
\hline High 7-Day Air & 32.8 & 1.5 & 29.7 & 36.9 & 48 \\
\hline Low Air & -22.9 & 4.2 & -32.8 & -15.6 & 47 \\
\hline Low Temperature Drop & 15.0 & 4.5 & 7.2 & 26.7 & 46 \\
\hline Degree Days Over $30^{\circ} \mathrm{C}$ & 86 & 59 & 14 & 248 & 48 \\
\hline
\end{tabular}

The air temperatures above are translated into pavement temperatures in the LTPPBind software. The LTPP algorithm gives pavement temperatures of $50.9^{\circ} \mathrm{C}$, high, and $-15.6^{\circ} \mathrm{C}$, low, for $50 \%$ reliability. The $98 \%$ reliability pavement temperatures, then, are $53.9^{\circ} \mathrm{C}$ and $-24.0^{\circ} \mathrm{C}$. For $98 \%$ reliability, LTPPBind calls for a PG58-28 grade binder.

The project is located in the Wet-Freeze region. A search of the NOAA records $(\underline{65})$ shows that the average annual precipitation level at this site is about $99 \mathrm{~cm}$ (39 inches) per year.

\subsection{Research Approach}

This section describes the field trial in more detail. Specific information about the materials and the mixtures used is also provided here. 


\subsubsection{Material Selection}

Seven different modifiers were selected for inclusion in this study. The specific materials selected are listed in Table 3. The modifiers selected were examples of the products that were under review at the time by the INDOT New Products Evaluation Committee. That committee was charged with reviewing new products and determining if the Department has a need for them, then conducting evaluations to determine if their performance is satisfactory. The committee had been approached by many modifier vendors and had very little guidance on how to select which modifiers to use.

Table 3 Modifiers Used in the Study

\begin{tabular}{|l|l|l|l|}
\hline Modifier & Supplier & Type of Modifier & $\begin{array}{l}\text { Polymer Content } \\
(\% \text { of binder })\end{array}$ \\
\hline Asphalt Rubber (AR) & Asphalt Rubber Systems & Wet Process Crumb Rubber & $20 \pm 3 \%$ \\
\hline $\begin{array}{l}\text { Multigrade Asphalt } \\
\text { Cement 20-40 (MGAC) }\end{array}$ & Asphalt Materials & Gelled Asphalt & NA \\
\hline Neoprene (Neo) & DuPont & Synthetic Rubber & $2 \%$ \\
\hline Novophalt (PE) & Novophalt America & Low density polyethylene & $5 \%$ \\
\hline Polyester Fibers (Fiber) & BoniFibers & Fiber & $*$ \\
\hline PAC20 (PAC) & $\begin{array}{l}\text { Styrelf (Now Koch } \\
\text { Materials) }\end{array}$ & Prereacted SB block & NA \\
\hline Cltrapave (SBR) & Textile Rubber & SBR Latex & $3 \%$ \\
\hline
\end{tabular}

*Fibers added at rate of $5 \mathrm{lbs} /$ ton for base and intermediate and $7.5 \mathrm{lbs} /$ ton of surface mixtures. $\mathrm{NA}=$ Not applicable to MGAC, Not available for PAC (proprietary information).

The first column includes the abbreviations used in this report to refer to the individual modifiers.

The SBR, PE, Neoprene and Asphalt Rubber all had an AC-10 base asphalt. The Fiber and Control sections used an AC-20. The base asphalts for the MGAC and PAC, which were provided as ready-to-use binders, were not specified. 


\subsubsection{Mix Designs}

All of the mixtures placed on the control and experimental sections were designed using the Marshall mix design that was the standard in Indiana at the time. Indiana designed their asphalt mixtures at $6 \%$ air voids, however, instead of the more common 3-5\% air voids used in most other states. For this reason, Indiana's Marshall mixes, in large part, met many of the requirements for Superpave mixtures, which were introduced in Indiana in 1993. Mixtures were also required to provide a minimum stability of $1200 \mathrm{lbs}$. and a flow value of between 6 and 16 .

For the most part, the mixture design process proceeded routinely. The majority of the mixtures were designed by Heritage Research Group, in Indianapolis. Some of the mixtures, as noted below, were designed elsewhere. The designs were 75-blow Marshall designs using handheld hammers. Mixes were oven-conditioned for one-hour prior to compaction, as required by INDOT practice at the time.

The final mix designs are shown in Tables 4 through 6 for the No. 5 base, No. 9 intermediate and No. 11 surface courses respectively. The gradations are shown in Tables 7 through 9 and graphically in Figures 4 through 6. These figures show the gradations of the experimental mixtures plotted against the Superpave control points for comparison purposes. The base mixtures essentially met the gradations for a $25 \mathrm{~mm}$ Superpave mixture, except for the asphalt rubber and polyethylene mixes, which were a little coarse between the $19 \mathrm{~mm}(3 / 4 \mathrm{in})$ and $150 \mu \mathrm{m}$ (\#100) sieves. The intermediate (formerly called binder) mixes largely conformed to the requirements of a 19mm Superpave mix. The Neoprene, MGAC, AR and SBR mixtures were on the coarse side from the $4.75 \mathrm{~mm}(\# 4)$ to $0.6 \mathrm{~mm}$ (\#30) sieves. (The intermediate mixtures could be considered $12.5 \mathrm{~mm}$ mixes based on $100 \%$ passing the $19 \mathrm{~mm}$ sieve, but then the mixes violate the gradation requirements significantly throughout the range of sizes.) The surface mixes met the gradation requirements of a Superpave $9.5 \mathrm{~mm}$ mix.

The mix designs were all quite similar except for the MGAC and asphalt rubber sections. The manufacturer maintained that MGAC was specifically designed to provide a thick film coating on the aggregates in open mixes. Since the additive was not designed to be used in typical dense graded mixes, the gradation was altered accordingly. The MGAC mixtures are therefore slightly more open than the other mixes, especially the base course. The gradation on the Asphalt Rubber section also had to be modified, especially in the finer sieve sizes, to accommodate the rubber particles. 
Table 4 No. 5 Base Course Mix Design Parameters

\begin{tabular}{|l|c|c|c|c|c|c|c|c|}
\hline Section & \%AC & Eff. AC\% & $\begin{array}{c}\text { VMA, } \\
\%\end{array}$ & $\begin{array}{c}\text { Air Voids, } \\
\%\end{array}$ & $\begin{array}{c}\text { Unit Weight, } \\
\mathrm{pcf}\end{array}$ & $\begin{array}{c}\text { Stability, } \\
\text { lbs. }\end{array}$ & $\begin{array}{c}\text { Flow, } \\
(0.01 \text { in })\end{array}$ & Max. Sp. Grav. \\
\hline A. Control & 4.1 & 4.0 & 13.8 & 6.0 & 146.5 & 2250 & 12.4 & 2.496 \\
\hline B. PAC & 4.2 & 4.0 & 13.7 & 5.7 & 146.9 & 3000 & 17.0 & 2.496 \\
\hline C. Fibers & 4.1 & 4.0 & 14.6 & 6.0 & 145.0 & 2000 & 15.0 & 2.486 \\
\hline D. PE & 4.2 & 4.0 & 14.8 & 6.0 & 145.1 & 2461 & 10.0 & 2.471 \\
\hline E. MGAC & 3.6 & 3.5 & 15.2 & $11.5 *$ & 143.7 & 2864 & 13.7 & 2.507 \\
\hline F. SBR & 4.1 & 4.0 & 13.2 & 6.0 & 147.4 & 2035 & 10.5 & 2.516 \\
\hline G. Neo & 4.1 & 4.0 & 13.8 & 6.0 & 146.5 & 1900 & 11.0 & 2.499 \\
\hline H. AR & $5.2^{* *}$ & $4.2^{* * *}$ & 14.0 & 3.0 & 147.5 & 2500 & 17.0 & 2.436 \\
\hline
\end{tabular}

*Air Voids calculated according to ASTM D3203.

**Asphalt Rubber content. Rubber content is $17 \%$ of AC- 10 .

$* * *$ Extracted asphalt content. 
Table 5 No. 9 Intermediate (Binder) Course Mix Design Parameters

\begin{tabular}{|l|c|c|c|c|c|c|c|c|}
\hline Section & $\%$ AC & Eff. AC\% & $\begin{array}{c}\text { VMA, } \\
\%\end{array}$ & $\begin{array}{c}\text { Air Voids, } \\
\%\end{array}$ & $\begin{array}{c}\text { Unit Weight, } \\
\mathrm{pcf}\end{array}$ & $\begin{array}{c}\text { Stability, } \\
\text { lbs. }\end{array}$ & $\begin{array}{c}\text { Flow } \\
(0.01 \text { in })\end{array}$ & Max. Sp. Grav. \\
\hline A. Control & 4.7 & 4.6 & 15.0 & 5.9 & 144.7 & 2440 & 9.2 & 2.464 \\
\hline B. PAC & 4.5 & 4.4 & 14.6 & 6.3 & 145.3 & 2650 & 14.0 & 2.485 \\
\hline C. Fibers & 4.7 & 4.6 & 14.8 & 6.0 & 145.3 & 2160 & 10.6 & 2.474 \\
\hline D. PE & 4.5 & 4.4 & 16.1 & 6.2 & 142.8 & 2325 & 10.7 & 2.441 \\
\hline E. MGAC & 4.5 & 4.4 & 15.2 & 6.2 & 144.6 & 3255 & 13.0 & 2.470 \\
\hline F. SBR & 4.5 & 4.4 & 15.2 & 6.2 & 144.5 & 3300 & 12.9 & 2.469 \\
\hline G. Neo & 4.7 & 4.6 & 14.8 & 6.0 & 145.2 & 2932 & 11.1 & 2.479 \\
\hline H. AR & $5.5^{* *}$ & $4.5^{* * *}$ & 14.5 & 3.1 & 147.0 & 2500 & 14.7 & 2.428 \\
\hline
\end{tabular}

*Air Voids calculated according to ASTM D3203.

**Asphalt Rubber content. Rubber content is $17 \%$ of AC- 10 .

***Extracted asphalt content. 
Table 6 No. 11 Surface Course Mix Design Parameters

\begin{tabular}{|l|c|c|c|c|c|c|c|c|}
\hline Section & \%AC & Eff. AC\% & $\begin{array}{c}\text { VMA, } \\
\%\end{array}$ & $\begin{array}{c}\text { Air Voids, } \\
\%\end{array}$ & $\begin{array}{c}\text { Unit Weight, } \\
\mathrm{pcf}\end{array}$ & $\begin{array}{c}\text { Stability, } \\
\mathrm{lbs}\end{array}$ & $\begin{array}{c}\text { Flow } \\
(0.01 \text { in })\end{array}$ & Max. Sp. Grav. \\
\hline A. Control & 5.7 & 5.5 & 16.2 & 5.4 & 144.6 & 2470 & 7.4 & 2.449 \\
\hline B. PAC & 6.3 & 5.9 & 16.7 & 6.0 & 142.0 & 2700 & 14.0 & 2.422 \\
\hline C. Fibers & 5.8 & 5.6 & 16.4 & 6.0 & 142.3 & 1900 & 11.0 & 2.430 \\
\hline D. PE & 5.8 & 5.5 & 17.4 & 6.0 & 140.9 & 2412 & 10.6 & 2.403 \\
\hline E. MGAC & 6.2 & 6.0 & 17.0 & 6.0 & 141.4 & 2850 & 9.0 & 2.412 \\
\hline F. SBR & 5.7 & 5.5 & 16.2 & 6.0 & 140.7 & 3216 & 10.5 & 2.402 \\
\hline G. Neo & 5.8 & 5.5 & 16.0 & 6.2 & 143.0 & 2360 & 9.9 & 2.444 \\
\hline H. AR & $7.2^{* *}$ & $5.7 * * *$ & 17.2 & 3.2 & 144.5 & 2550 & 17.0 & 2.389 \\
\hline
\end{tabular}

*Air Voids calculated according to ASTM D3203.

**Asphalt Rubber content. Rubber content is $17 \%$ of AC- 10 .

$* * *$ Extracted asphalt content. 
Table 7 No. 5 Base Course Mix Design Gradations

\begin{tabular}{|l|c|c|c|c|c|c|c|}
\hline \multirow{2}{*}{ Section } & \multicolumn{7}{|c|}{ Percent Passing Sieve Size } \\
\cline { 2 - 8 } & $37.5 \mathrm{~mm}(1.5 \mathrm{in})$ & $25 \mathrm{~mm}(1 \mathrm{in})$ & $19 \mathrm{~mm}(3 / 4 \mathrm{in})$ & $12.5 \mathrm{~mm}(1 / 2 \mathrm{in})$ & $4.75 \mathrm{~mm}(\# 4)$ & $(\# 30)$ & $75 \mu \mathrm{m}(\# 200)$ \\
\hline A. Control & $100 \%$ & 95.0 & 79.6 & 61.6 & 34.0 & 8.7 & 3.8 \\
\hline B. PAC & $100 \%$ & 95.0 & 80.0 & 61.0 & 34.0 & 9.0 & 3.6 \\
\hline C. Fibers & $100 \%$ & 95.0 & 81.7 & 62.1 & 34.7 & 7.7 & 3.2 \\
\hline D. PE & $100 \%$ & 94.4 & 79.5 & 60.1 & 28.5 & 7.9 & 3.1 \\
\hline E. MGAC & $100 \%$ & 96.6 & 75.7 & 51.1 & 20.8 & 4.5 & 2.2 \\
\hline F. SBR & $100 \%$ & 95.0 & 80.8 & 61.5 & 35.0 & 7.7 & 3.2 \\
\hline G. Neo & $100 \%$ & 95.0 & 80.8 & 61.5 & 35.0 & 7.7 & 3.2 \\
\hline H. AR & $100 \%$ & 91.9 & 78.5 & 58.5 & 34.0 & 7.5 & 3.5 \\
\hline
\end{tabular}


Table 8 No. 9 Intermediate (Binder) Course Mix Design Gradations

\begin{tabular}{|l|c|c|c|c|c|c|c|}
\hline \multirow{2}{*}{ Section } & \multicolumn{7}{|c|}{ Percent Passing Sieve Size } \\
\cline { 2 - 8 } & $37.5 \mathrm{~mm}(1.5 \mathrm{in})$ & $25 \mathrm{~mm}(1 \mathrm{in})$ & $19 \mathrm{~mm}(3 / 4 \mathrm{in})$ & $12.5 \mathrm{~mm}(1 / 2 \mathrm{in})$ & $4.75 \mathrm{~mm}(\# 4)$ & $(\# 30)$ & $75 \mu \mathrm{m}(\# 200)$ \\
\hline A. Control & $100 \%$ & $100 \%$ & $100 \%$ & 84.9 & 42.8 & 10.9 & 3.7 \\
\hline B. PAC & $100 \%$ & $100 \%$ & $100 \%$ & 86.0 & 25.0 & 9.5 & 3.7 \\
\hline C. Fibers & $100 \%$ & $100 \%$ & $100 \%$ & 86.2 & 43.9 & 9.3 & 3.3 \\
\hline D. PE & $100 \%$ & $100 \%$ & $100 \%$ & 85.4 & 39.8 & 6.5 & 2.9 \\
\hline E. MGAC & $100 \%$ & $100 \%$ & $100 \%$ & 85.0 & 33.1 & 3.8 & 3.2 \\
\hline F. SBR & $100 \%$ & $100 \%$ & $100 \%$ & 85.0 & 33.1 & 7.8 & 3.0 \\
\hline G. Neo & $100 \%$ & $100 \%$ & $100 \%$ & 86.0 & 43.2 & 6.1 & 2.9 \\
\hline H. AR & $100 \%$ & $100 \%$ & $100 \%$ & 84.9 & 41.2 & 7.9 & 3.6 \\
\hline
\end{tabular}


Table 9 No. 11 Surface Course Mix Design Gradations

\begin{tabular}{|l|c|c|c|c|c|c|c|}
\hline \multirow{2}{*}{ Section } & \multicolumn{7}{|c|}{ Percent Passing Sieve Size } \\
\cline { 2 - 8 } & $37.5 \mathrm{~mm}(1.5 \mathrm{in})$ & $25 \mathrm{~mm}(1 \mathrm{in})$ & $19 \mathrm{~mm}(3 / 4 \mathrm{in})$ & $12.5 \mathrm{~mm}(1 / 2 \mathrm{in})$ & $4.75 \mathrm{~mm}(\# 4)$ & $(\# 30)$ & $75 \mu \mathrm{m}(\# 200)$ \\
\hline A. Control & $100 \%$ & $100 \%$ & $100 \%$ & $100 \%$ & 62.1 & 21.9 & 3.9 \\
\hline B. PAC & $100 \%$ & $100 \%$ & $100 \%$ & $100 \%$ & 52.0 & 18.0 & 2.9 \\
\hline C. Fibers & $100 \%$ & $100 \%$ & $100 \%$ & $100 \%$ & 63.9 & 17.5 & 2.7 \\
\hline D. PE & $100 \%$ & $100 \%$ & $100 \%$ & $100 \%$ & 61.0 & 13.2 & 3.2 \\
\hline E. MGAC & $100 \%$ & $100 \%$ & $100 \%$ & $100 \%$ & 52.7 & 15.9 & 2.5 \\
\hline F. SBR & $100 \%$ & $100 \%$ & $100 \%$ & $100 \%$ & 57.4 & 14.6 & 3.3 \\
\hline G. Neo & $100 \%$ & $100 \%$ & $100 \%$ & $100 \%$ & 57.4 & 14.6 & 3.2 \\
\hline H. AR & $100 \%$ & $100 \%$ & $100 \%$ & $100 \%$ & 64.4 & 17.1 & 3.7 \\
\hline
\end{tabular}




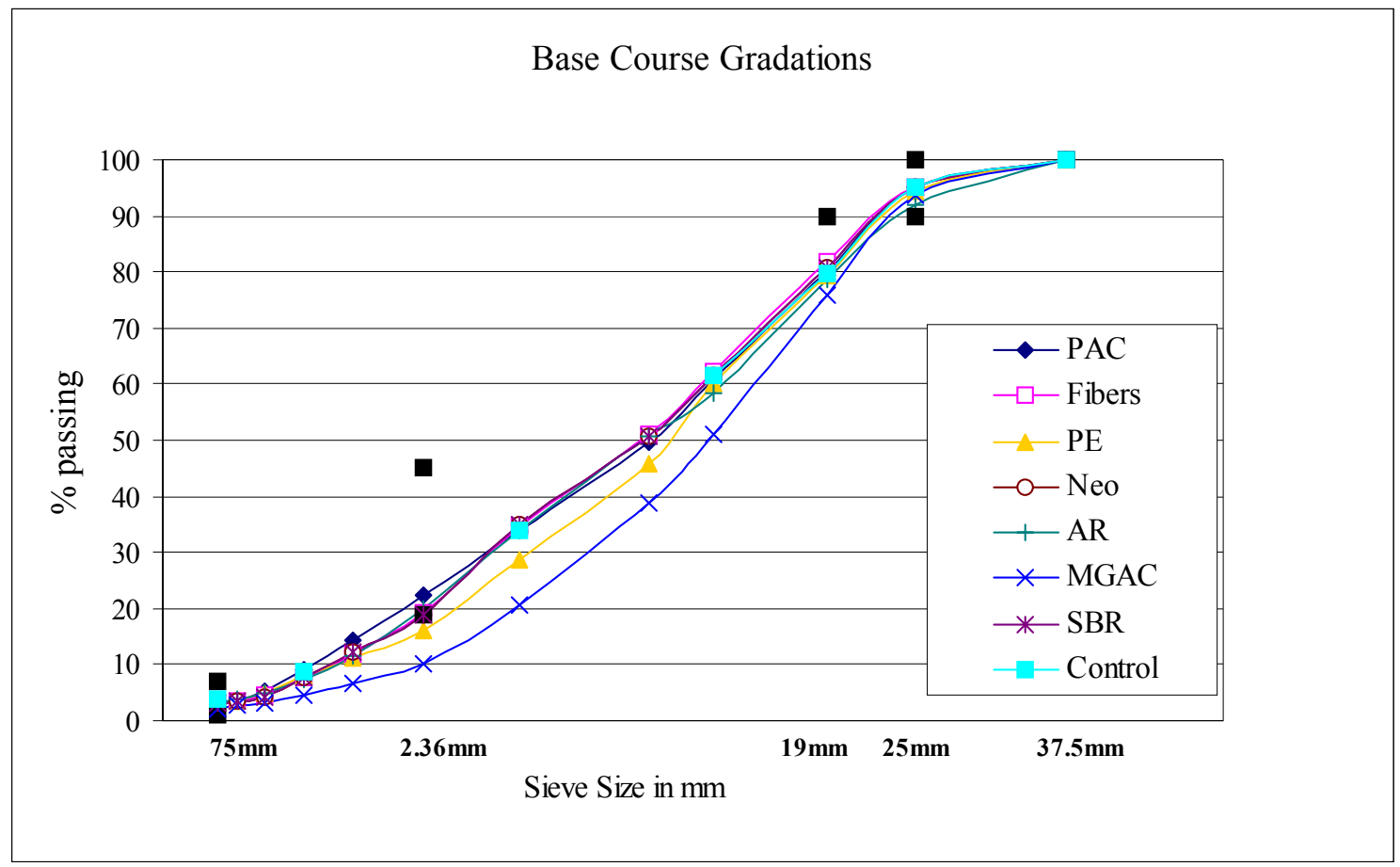

Figure 4 Base Course Gradations

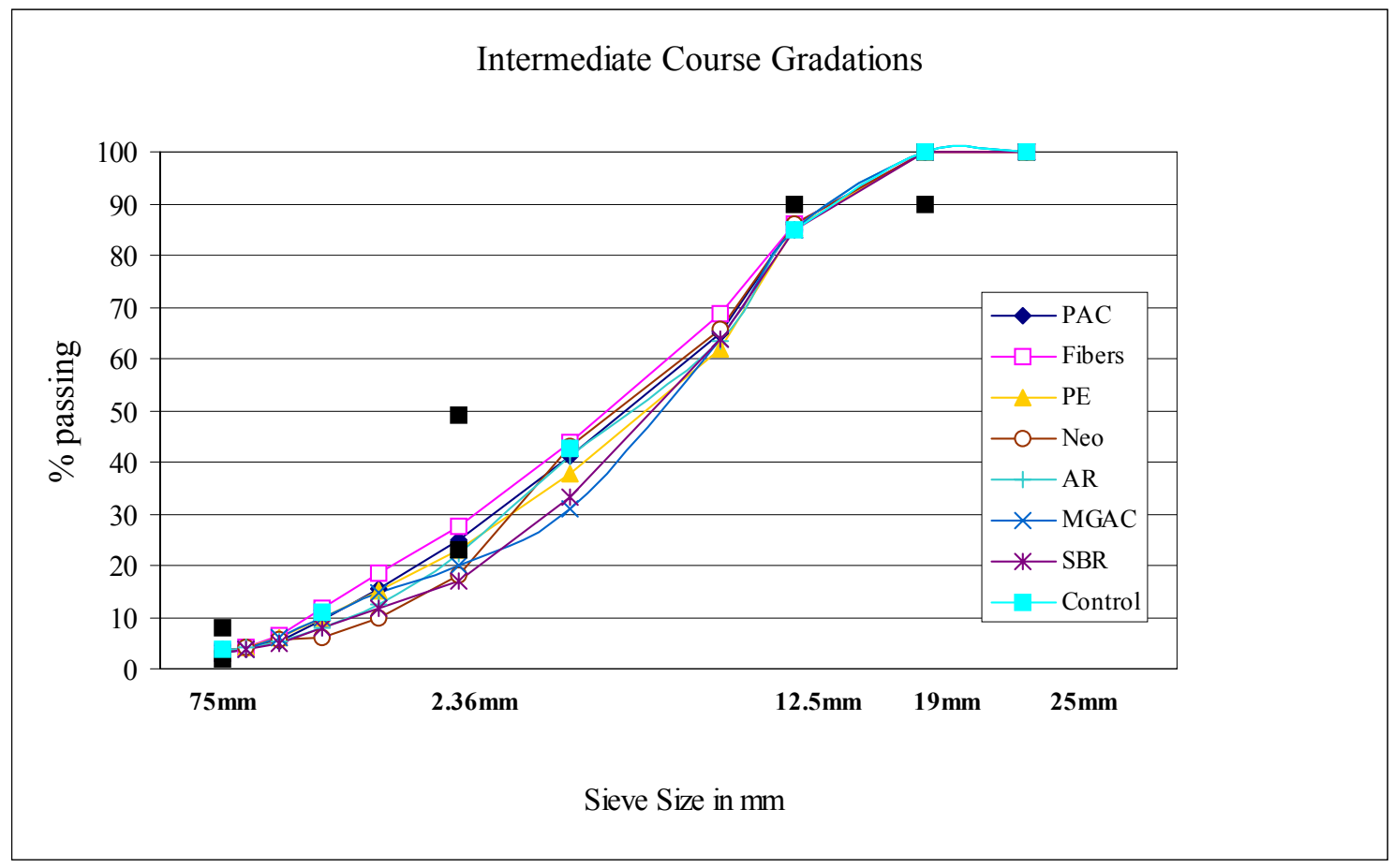

Figure 5 Intermediate Course Gradations 


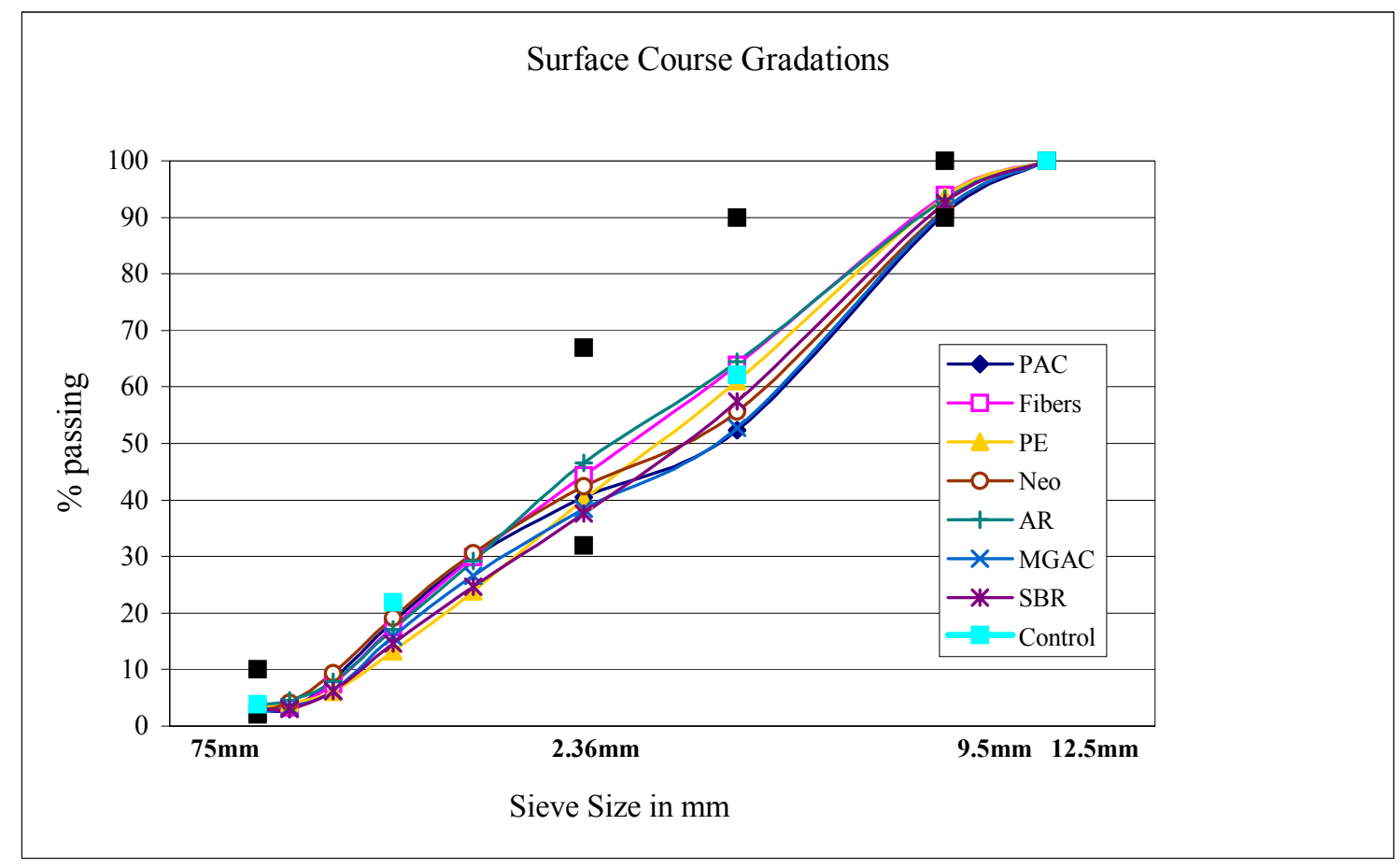

Figure 6 Surface Course Gradations

All the aggregates in all the mixtures were obtained from the same sources and the same sizes of aggregates were blended, though in slightly different proportions. The limestone coarse and fine aggregates used in the base and intermediate courses were all obtained from the MartinMarietta Kentucky Avenue quarry in Indianapolis. For the surface course, INDOT friction policies led to the use of a 50-50 blend of high quality dolomite from the Delphi Quarry and slag from Levy Slag for the coarse aggregates. Manufactured coarse flume sand and up to $20 \%$ natural sand, both from Martin-Marietta, were the fine aggregates used in the surface mixes. All of the aggregates, except for the natural sand, were $100 \%$ crushed materials and are locally recognized as high quality aggregates.

\subsubsection{Comments on Individual Mix Designs}

As noted above, most of the mix designs were relatively routine and were performed by Heritage Research. Heritage designed the mixtures with MGAC, SBR, Neoprene, Polyethylene and Fibers. Some of the more unique designs are discussed individually below. 
The asphalt rubber mix design was completed by Crafco, Inc., in Chandler, Arizona, for the asphalt rubber supplier, Asphalt Rubber Systems. The base asphalt for this project was an AC-10 supplied by Asphalt Materials, Inc., of Indianapolis. Conventional practice calls for using binder one grade softer than normal to account for the stiffening effect of the crumb rubber. An extender oil was also added at $4 \%$ by weight of binder. The final blended binder, then, consisted of $79 \%$ AC- $10,4 \%$ extender oil and $17 \%$ rubber by weight. The rubber portion was comprised of $14 \%$ ambient ground tire rubber and 3\% other rubber. The other rubber was reported to contain ground tennis and racquetball balls, to provide more resilience or "bounce."

Crafco indicated that designing asphalt rubber mixes at $6.0 \%$ air voids would result in a dry mix with insufficient binder content. Because the rubber particles are considered part of the binder, the mixes are typically designed at the low end of the allowable air void level to allow for the void filling effect of the rubber particles and still maintain an adequate binder content. The asphalt rubber mixes, therefore, were designed at 3-4\% air voids. The flow values were also increased because asphalt rubber mixes tend to exhibit higher flow values due to the elastomeric behavior of the rubber particles.

The Styrelf (PAC) mixtures were all designed by Elf Asphalt at their Terre Haute, Indiana, laboratory. No unique changes in the mixture were required. 


\section{CONSTRUCTION DETAILS}

This chapter describes the construction of the test sections, summarizes the quality control testing during construction, and details testing issues related to the various additives studied.

Due to traffic considerations on this heavily traveled urban interstate, all lane closures and paving were done at night. Lanes could be closed for construction between 6 p.m. and 5 a.m. The Reith-Riley Construction Company, Inc., completed paving of the test sections in July, August and September of 1990.

Following undersealing, patching of the existing jointed PCC pavement, and installation of edgedrains, three lifts of bituminous mix were placed as shown in the cross section in Figure 3. In general, the base and intermediate courses were placed on subsequent nights in July or August. The surface mixtures were placed between September 5 and 12, 1990, after incidental median work was completed. An asphalt emulsion tack coat (AE-T) was used on the concrete and between lifts according to standard INDOT practice.

Traffic was diverted to the right hand lane, the driving lane, while the inner shoulder and high speed lane were paved in one $4.9 \mathrm{~m}$ (16 ft.) pass. The center lane was then paved in a $3.6 \mathrm{~m}$ (12-ft.) pass. After traffic was diverted onto the new pavement in the high speed lane, the driving lane was paved.

Construction was accomplished with one Cedarrapids CR461 paver, two to three tandem rollers, and a broom truck and a tack distributor as needed. The number of haul trucks varied from four to ten. The plant used was a 6-ton H\&B batch plant with a baghouse and storage silos (Figure 7). 


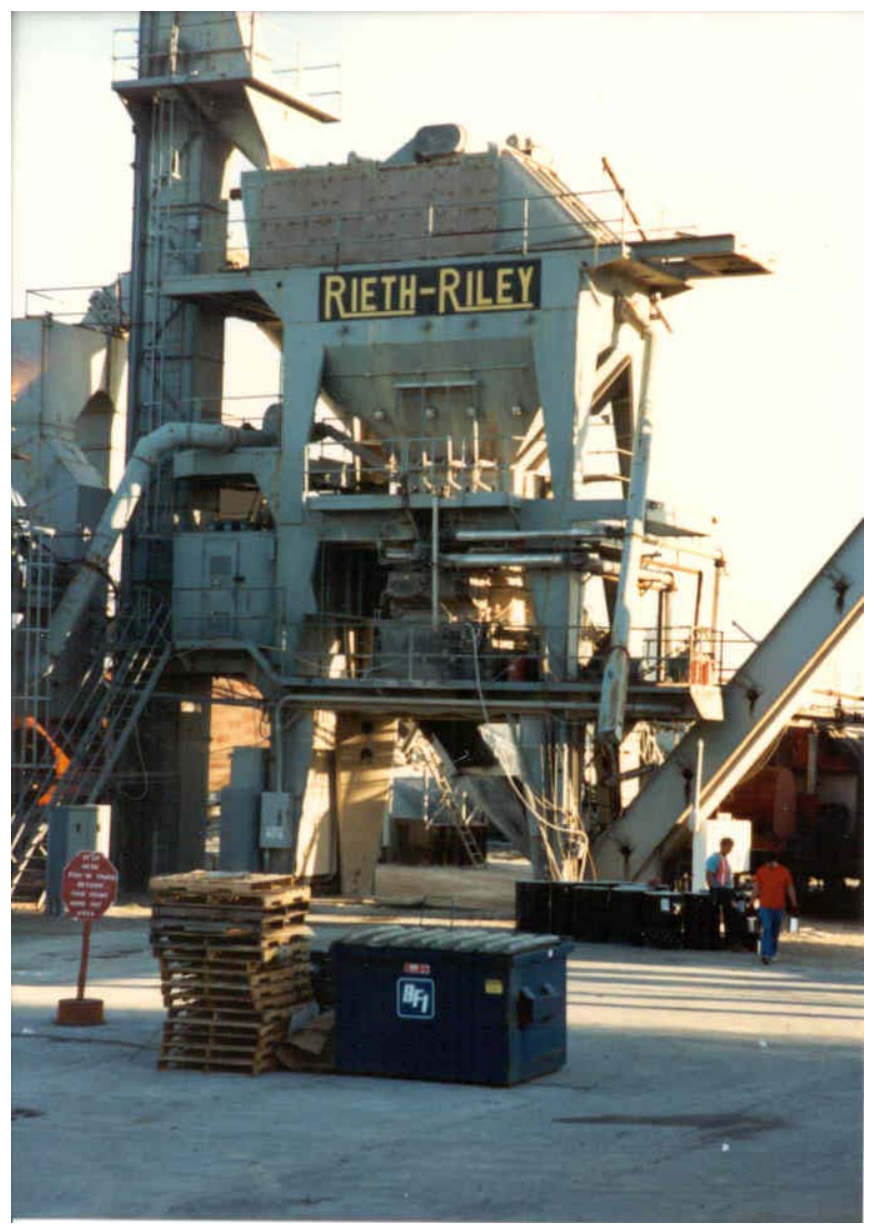

Figure 7 Hot Mix Batch Plant Used on Project

\subsection{Construction Quality Testing}

Concerns were expressed by INDOT personnel prior to construction that routine construction quality control tests might not be applicable to the modified mixtures.

Consequently, a few concessions were made, but for the most part they were relatively minor.

A conventional vacuum extraction test, as was routine at the time, could not be used with the PAC. Reflux extractions could be done using limonene or trichloroethylene. The binder contents of the PAC mixtures were therefore determined by reflux extractions done at the INDOT Materials and Tests lab. Normal binder and mix sampling techniques could be used. 
When doing an extraction on the asphalt rubber mixes, the rubber remains behind with the aggregate. The binder content measured, then, is the actual asphalt content, not the asphalt rubber content. The DOT agreed to waive gradation testing of the asphalt rubber mixes since there was no practical way to account for the rubber particles in the gradation or to separate the rubber from the aggregates. The contractor was, however, required to perform a hot bin analysis to assure that the gradation requirements were being met going into the hopper of the plant.

No problems were noted with using the nuclear gauge to check density on any of the experimental mixtures.

Standard INDOT practice at the time also required compaction of Marshall specimens at the plant during production. These specimens were then checked for volumetrics, Marshall stability and flow. No problems were encountered in preparing Marshall specimens except for some swelling of the asphalt rubber specimens after compaction. The results of the construction quality control tests are summarized in Table 10.

Table 10 Construction Quality Test Results

\begin{tabular}{|c|c|c|c|c|c|c|c|}
\hline Modifier & Layer & $\begin{array}{c}\% \\
\text { Bitumen }\end{array}$ & $\begin{array}{c}\text { Average } \\
\text { BSG }\end{array}$ & MSG & $\begin{array}{c}\text { Average } \\
\text { \% Voids }\end{array}$ & $\begin{array}{c}\text { Average } \\
\text { Stability, } \\
\text { lbs. }\end{array}$ & $\begin{array}{c}\text { Average } \\
\text { Flow, } \\
0.01 \text { in. }\end{array}$ \\
\hline PAC & Base & 4.05 & 2.407 & 2.448 & 1.7 & 3313 & 17.7 \\
\hline & Intermed & 4.1 & 2.371 & 2.485 & 4.6 & 2839 & 20.5 \\
\hline & Surf & 6.0 & 2.368 & 2.434 & 2.7 & 2911 & 15.5 \\
\hline Fiber & Base & -- & 2.396 & 2.482 & 3.5 & 2758 & 26.7 \\
\hline & Intermed & -- & 2.387 & 2.451 & 2.6 & 2549 & 18.7 \\
\hline & Surf & 5.9 & 2.308 & 2.435 & 5.2 & 2107 & 14.7 \\
\hline PE & Base & 3.3 & 2.384 & 2.481 & 3.9 & 2387 & 14.5 \\
\hline & Intermed & 4.1 & 2.356 & 2.463 & 4.4 & 2398 & 18.7 \\
\hline & Surf & 5.3 & 2.360 & 2.450 & 3.7 & 4208 & 13.8 \\
\hline MGAC & Base & -- & 2.368 & 2.511 & 5.7 & 1936 & 19.3 \\
\hline & Intermed & -- & 2.363 & 2.464 & 4.1 & 2025 & 15.3 \\
\hline SBR & Surf & 6.0 & 2.358 & 2.440 & 3.3 & 2393 & 11.7 \\
\hline & Base & 3.95 & 2.426 & 2.465 & 1.6 & 2803 & 22.3 \\
\hline & Intermed & 4.1 & 2.332 & 2.478 & 5.9 & 2424 & 23.5 \\
\hline Neo & Surf & 5.45 & 2.334 & 2.453 & 4.9 & 2236 & 20.2 \\
\hline & Base & 3.55 & 2.392 & 2.495 & 4.1 & 2632 & 20.2 \\
\hline & Intermed & 4.0 & 2.357 & 2.454 & 4.0 & 2508 & 20.8 \\
\hline & Surf & 5.2 & 2.352 & 2.462 & 4.5 & 2617 & 11.5 \\
\hline AR & Base & 4.25 & 2.355 & 2.440 & 3.6 & 2073 & 20.7 \\
\hline & Intermed & 4.6 & 2.356 & 2.394 & 1.6 & 2363 & 20.2 \\
\hline & Surf & 6.2 & 2.273 & 2.408 & 5.6 & 1627 & 15.0 \\
\hline
\end{tabular}

-- Results not available, $\mathrm{BSG}=$ bulk specific gravity and $\mathrm{MSG}=$ maximum theoretical specific gravity 


\subsubsection{Stability and Flow Values}

The stability and flow values measured on Marshall specimens compacted at the plant are summarized in Table 10. This section briefly discusses those results.

All of the plant-produced mixtures provided a stability greater than the minimum value of $1200 \mathrm{lbs}$. The lowest value was 1,627 lbs. for the asphalt rubber surface course, and the highest value was more than two and a half times greater, i.e. 4,208 lbs., for the Novophalt (PE) surface. In general, within a binder type, the specimens with a lower air void content showed the highest stability, and the specimens with the highest air voids showed the lowest stability, as expected.

During construction, every experimental binder exhibited a flow value higher than its design flow value and higher than the acceptable range of 6 to 16 for at least one mixture type (base, intermediate or surface). The highest value recorded was 26.7 for the fiber base course. All of the SBR mixtures had flow values in excess of 20. The asphalt rubber mixtures had average flow values of 20.7, 20.2 and 15.0 for the base, intermediate and surface, respectively. The mix designers had pointed out that asphalt rubber mixtures typically do show high flow values due to the elastometric properties of the rubber particles. None of the other modifier suppliers indicated this was the case for their products, and the design values were not excessive. The fact that the air voids of the plant samples were lower than design may explain some, but not all, of these results. The only other apparent explanation for flow values being higher for the plant mix than for design is that the design mixtures were held for one hour in an oven prior to compaction. Plant mix samples were collected directly from the trucks at the plant. It is possible that the oven conditioning required for design artificially aged the mixture and led to lower flow values.

In any case, none of these mixtures have exhibited rutting problems in the field, despite their high flow values. This demonstrates one of the shortcomings of the Marshall mix design system for today's materials and traffic conditions. High flow values are not reliable indicators of potential problems, nor are high stability values an assurance that mixtures will not rut. 


\subsubsection{Gradation and Binder Content Results}

At the time of this project, INDOT tested binder content by vacuum extraction and then checked the aggregate gradation versus the job mix formula. The gradation and binder content were controlled based on two considerations. Did the value fall within the ranges allowed on gradation and binder content, and was the difference between replicate test results within an allowable range? Adjustment points were assessed for material quality based on these comparisons and on field density as discussed in the next section. The points for mix quality and density were added together and applied as a deduction to the price per ton for that lot of mix. (Deductions for failed materials, noted in section 3.2, were applied over and above these adjustment points.) Perusal of the adjustment points assessed therefore gives a good indication of the quality and consistency of the materials produced.

All of the mixtures produced passed the standard quality control tests, except as noted in this section. Overall, the mixes were produced as designed. No adjustment points were assessed for excessive variability between replicate samples. The exceptions noted here are all relatively minor, indicating the mix quality was not seriously compromised.

Mixture quality adjustment points were applied to two out of six lots of the control base mix, two of four lots of control intermediate course mix and all four lots of surface mix. The adjustment points were relatively minor, resulting in a deduction of less than $2 \%$ of the contract price in most cases and a maximum of about a 5\% deduction for one lot of intermediate mix. All of these were due to deviations of the aggregate gradation from the job mix formulae.

Minor adjustment points for gradation were assessed on the base with fibers (2.4\%), the surface with fibers $(0.6 \%)$, the surface with PE $(0.2 \%)$ and the base with SBR $(2.7 \%)$. The greatest deduction on gradation was assessed against the surface with SBR. A 12.6\% reduction in pay was due to the gradation being too coarse on the $12.5 \mathrm{~mm}(1 / 2 \mathrm{in}$.) and $4.75 \mathrm{~mm}(\# 4)$ sieves. Only $45 \%$ passed the $4.75 \mathrm{~mm}$ sieve versus $51.7-67 \%$ passing called for in the job mix formula. A minor adjustment of $2.4 \%$ was made on the surface with MGAC; the documentation on the reason for this is not available.

No mixture quality adjustment points were applied to the PAC, Neoprene or rubber mixes. Rubber mixes were checked for binder content only due to the rubber particles interfering with the gradation analysis. 


\subsubsection{Density Testing Results}

INDOT also used adjustment points to assess penalties for failing to achieve adequate compaction in the field. A control strip procedure was used to establish the target density during construction. For each lift of each mixture placed, the contractor would continue rolling the mix until the density peaked out, as determined by nuclear gauge. Ten cores would be pulled from each test strip after the density had peaked. The average density of the cores was set as the target density for that lift. These values are shown in Table 11. The contractor was required to achieve $98 \%$ of the target density or adjustment points would be assessed. A nuclear gauge was then used throughout construction to control the roller operations and verify that the target density had been achieved. This procedure was used on all the mixes except the Multigrade base mix. On that open graded mix, the manufacturer recommended the number of roller passes to apply.

Density was achieved in all cases except for one lot (3,034 tons) of the control intermediate course. The adjustment for that one lot was $0.9 \%$, indicating the contractor achieved $97.1 \%$ of the job mix density.

Table 11 Test Strip Core Density

\begin{tabular}{|c|c|c|c|}
\hline \multirow{2}{*}{ Mix Type } & \multicolumn{3}{|c|}{ Average Density, pcf } \\
\cline { 2 - 4 } & Surface & Intermediate & Base \\
\hline PE & 140.3 & 141.4 & 145.3 \\
\hline AR & 136.5 & 142.2 & 146.2 \\
\hline MGAC & 140.3 & 141.5 & N/A \\
\hline Fiber & 134.8 & 138.4 & 146.0 \\
\hline PAC & 138.6 & 140.7 & 143.5 \\
\hline Neo & 138.9 & 138.4 & 143.8 \\
\hline SBR & 142.4 & 139.2 & 144.3 \\
\hline Control & 137.0 & 141.5 & 145.0 \\
\hline
\end{tabular}




\subsection{Discussion of Construction}

Construction went smoothly, for the most part, except as noted below. The following sections describe how the individual mixtures were produced and any unusual occurrences or observations noted during production and construction. The quantities of each material produced are listed in Table 12. Sufficient quantities of each mix were produced to allow the plant and paving operations to stabilize after start-up.

Table 12 Quantities of Mix Placed

\begin{tabular}{|l|c|c|c|}
\hline Mixture & Base (tons) & Intermediate (tons) & Surface (tons) \\
\hline Control & $15,698.5$ & $8,172.6$ & $8,791.0$ \\
\hline PAC & $1,771.5$ & 818.4 & 914.5 \\
\hline Fibers & $1,588.9$ & $1,781.7$ & 804.0 \\
\hline Novophalt (PE) & $1,948.9$ & 975.7 & 907.7 \\
\hline Multigrade & $1,765.9$ & 859.5 & 856.9 \\
\hline SBR & $1,774.3$ & 810.6 & 908.6 \\
\hline Neoprene & $1,704.5$ & 731.6 & 877.4 \\
\hline Asphalt Rubber & $1,744.2$ & $1,054.1$ & 930.8 \\
\hline
\end{tabular}

\subsubsection{Conventional Mixtures}

No particular problems were noted during production or construction of the control sections.

\subsubsection{Styrelf (PAC)}

Paving with the PAC base material began July $24^{\text {th }}$. This was the first experimental material used on the project. The intermediate course was placed July $25^{\text {th }}$ and the surface course followed on September $10^{\text {th }}$. 
The PAC additive was premixed in the asphalt. The binder was simply pumped into the plant as usual (Figure 8). A definite odor was noted with this material, but it was not offensive and no worker complaints were noted.

Material testing of the binder showed that, in some cases, the binder used in the base and intermediate courses failed to meet the specified original penetration requirements. The specifications required a penetration at $25^{\circ} \mathrm{C}\left(77^{\circ} \mathrm{F}\right)$ of between $45-75$, and the actual measured penetrations were in the range of 78-80. The base and intermediate mixtures were allowed to remain in place with a deduction in price. Deductions of $25 \%$ to $50 \%$ of the invoice price were applied to 114.2 tons of mix.

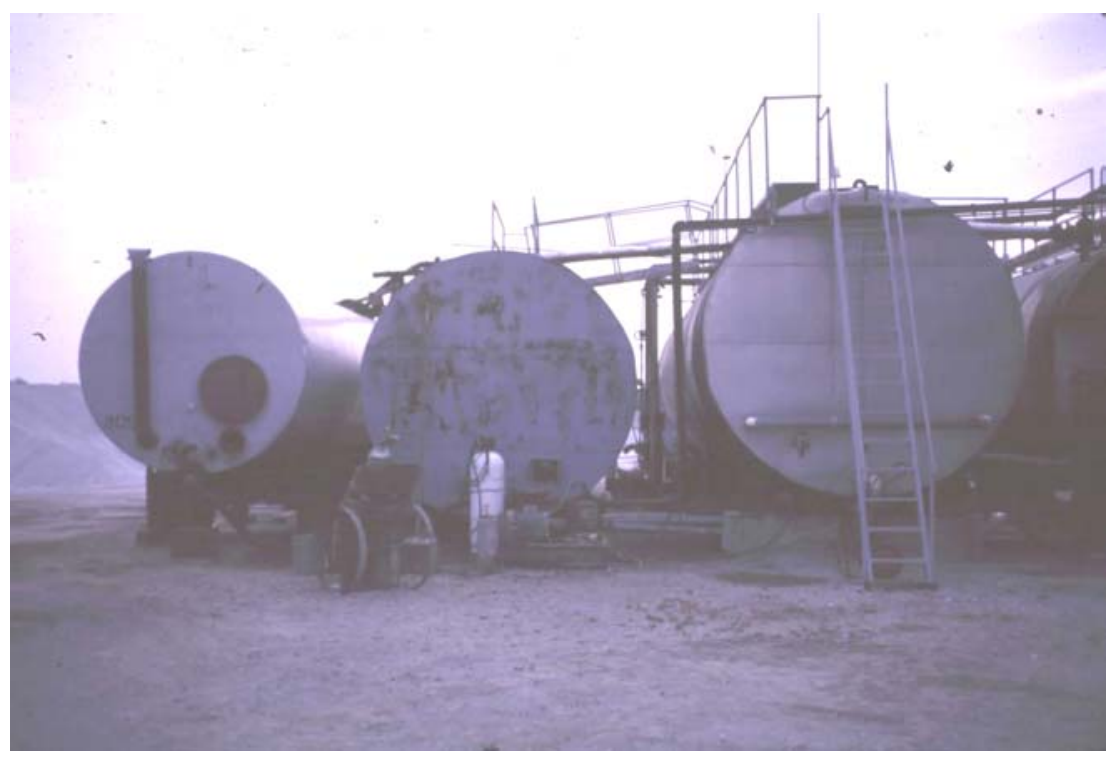

Figure 8 Neoprene, MGAC and PAC Were Metered into AC Line at Plant

\subsubsection{Polyester Fibers}

Paving with the fiber modified base course began July $26^{\text {th }}$, but was halted after the high speed lane was paved due to a breakdown at the plant. Placing the base mix resumed and was completed July $28^{\text {th }}$. At that time a paver malfunction caused a dip in the base between stations $118+00$ and $117+00$. The contractor wedged this dip with the fiber modified intermediate course, placed on July $30^{\text {th }}$. The surface was placed on September $6^{\text {th }}$. 
When production of the fiber base began, the contractor was using 30 seconds of dry mixing time to distribute the fibers followed by 35 seconds of wet mixing time. The fiber representative observed a fairly large amount of fines being produced during the dry mixing and recommended shortening the dry mixing time to 10 seconds with a 35 second wet mixing time. This appeared to provide adequate distribution of the fibers without producing excess fines. This mixing sequence was used for the remainder of the fiber mixes.

The fibers were delivered in plastic bags. The bags were delivered to the weigh hopper via conveyor (Figure 9) and the required number of bags was tossed into the hopper for each batch. The bags melted when they came in contact with the heated aggregates.

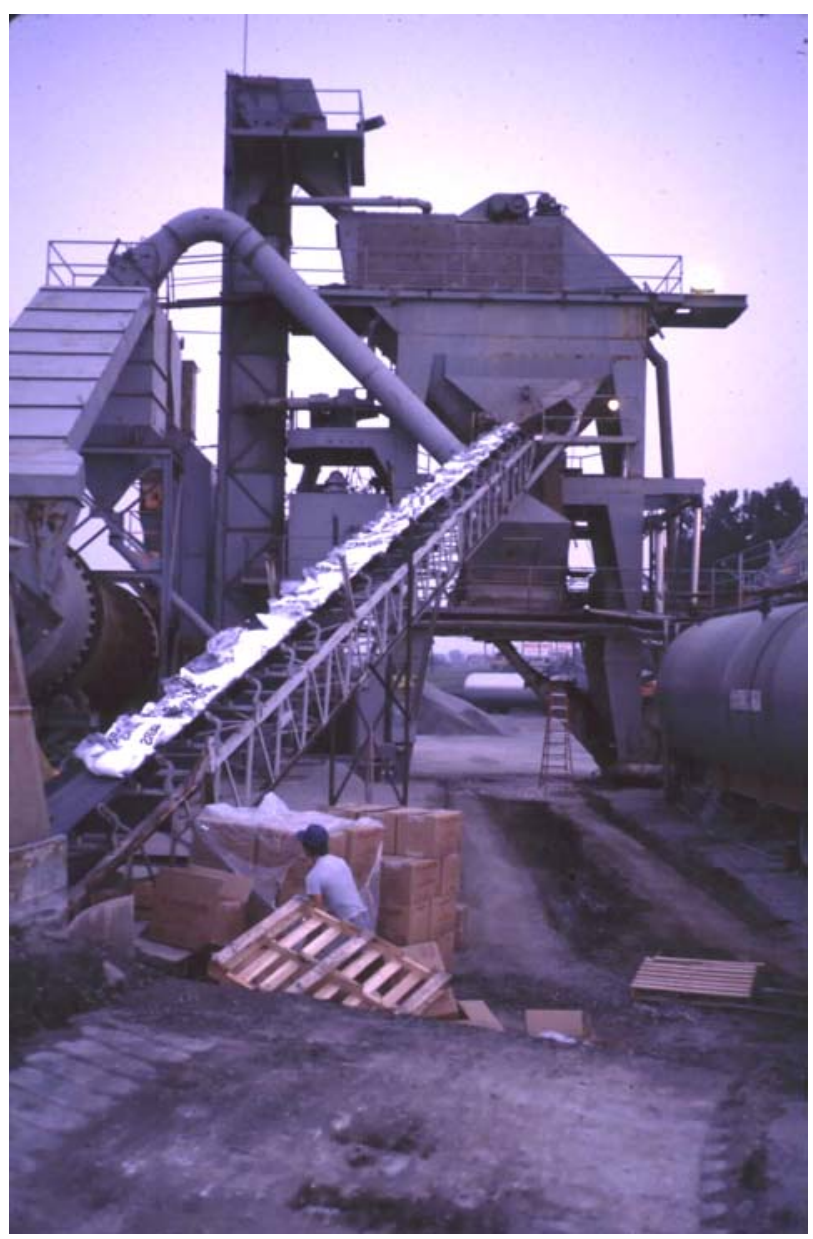

Figure 9 Fibers in Bags on Conveyor to Weigh Hopper 


\subsubsection{Novophalt}

The modifier supplier, Novophalt America, provided a special mixing unit (Figure 10) to shear the polyethylene pellets into the binder. The blended binder was then pumped into the plant for production. The base course was placed on July $31^{\text {st }}$, the intermediate on August $1^{\text {st }}$ and the surface on September $7^{\text {th }}$.

The only problem noted during construction was that the fins separating the fine and coarse aggregate bins were not high enough, allowing the possibility of aggregates falling into the wrong bin. This may be the cause of the slight penalty applied to the PE surface course gradation.

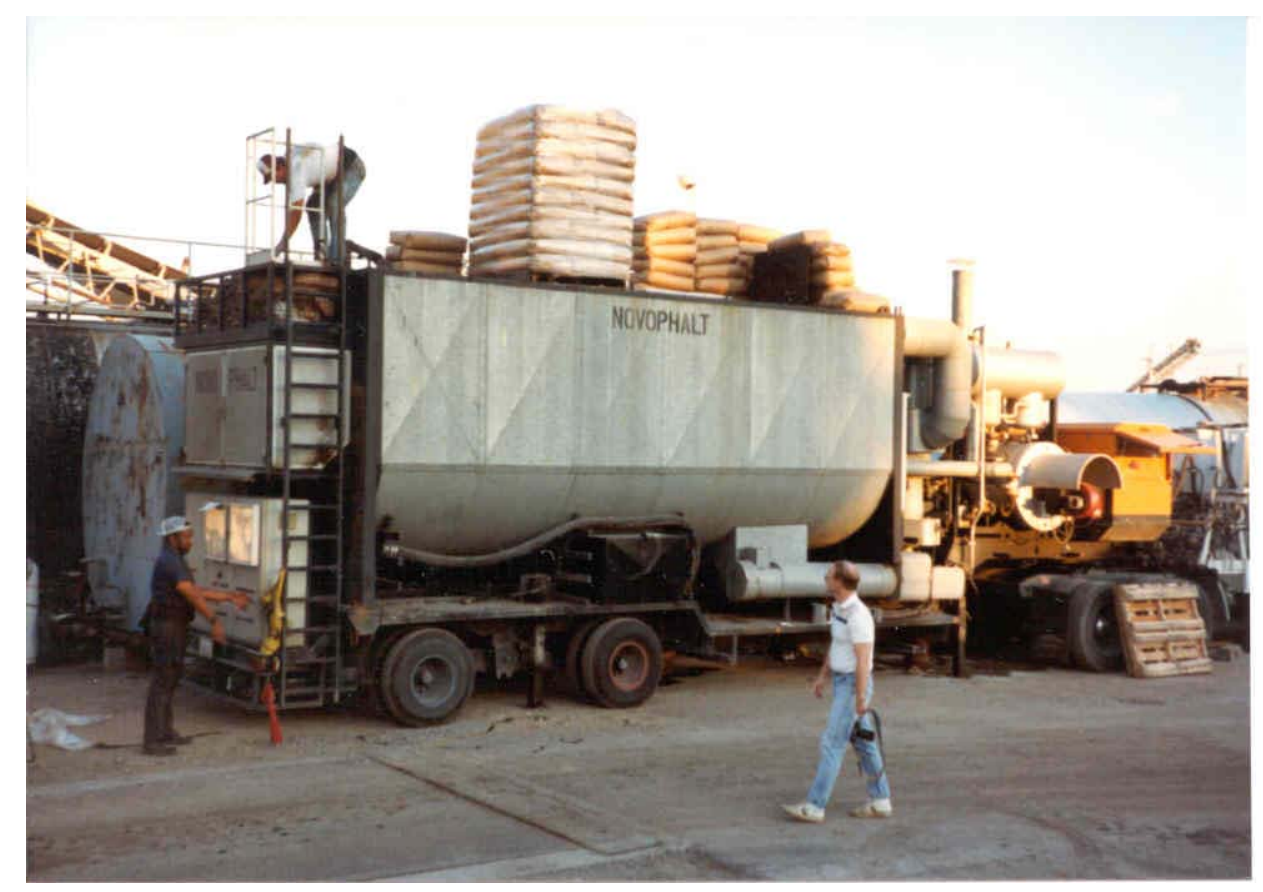

Figure 10 The Novophalt Mobile Mixing Unit

\subsubsection{Multigrade Asphalt Cement}

The Multigrade asphalt cement (MGAC) base course was produced and placed August $2^{\text {nd }}$ and the intermediate course was placed on August $3^{\text {rd }}$. Placement of the surface course began 
on September $8^{\text {th }}$, but was halted due to rain after the high speed and center lanes were paved. The driving lane was paved September $10^{\text {th }}$.

The MGAC was also a ready-to-use binder, so no special provisions were needed to add this at the plant (Figure 8).

Samples of the MGAC taken on August $3^{\text {rd }}$ failed to meet the specified softening point value of $140^{\circ} \mathrm{F}$ minimum. The measured softening point was $137^{\circ} \mathrm{F}$. The morning sample on that day also failed to meet the specified thin film oven test (TFOT) viscosity of 4,000 poise minimum; the measured value was 3,750 poise. The material was allowed to remain in place with $5-10 \%$ penalties assessed on various lots totaling 43.6 tons of mix.

\subsubsection{Styrene Butadiene Rubber (SBR)}

The SBR latex was supplied in 55-gallon barrels. A hose and pump were used to meter the SBR directly into the asphalt line feeding the mixing unit, as shown in Figures 11 and 12. No particular problems were noted with this technique.

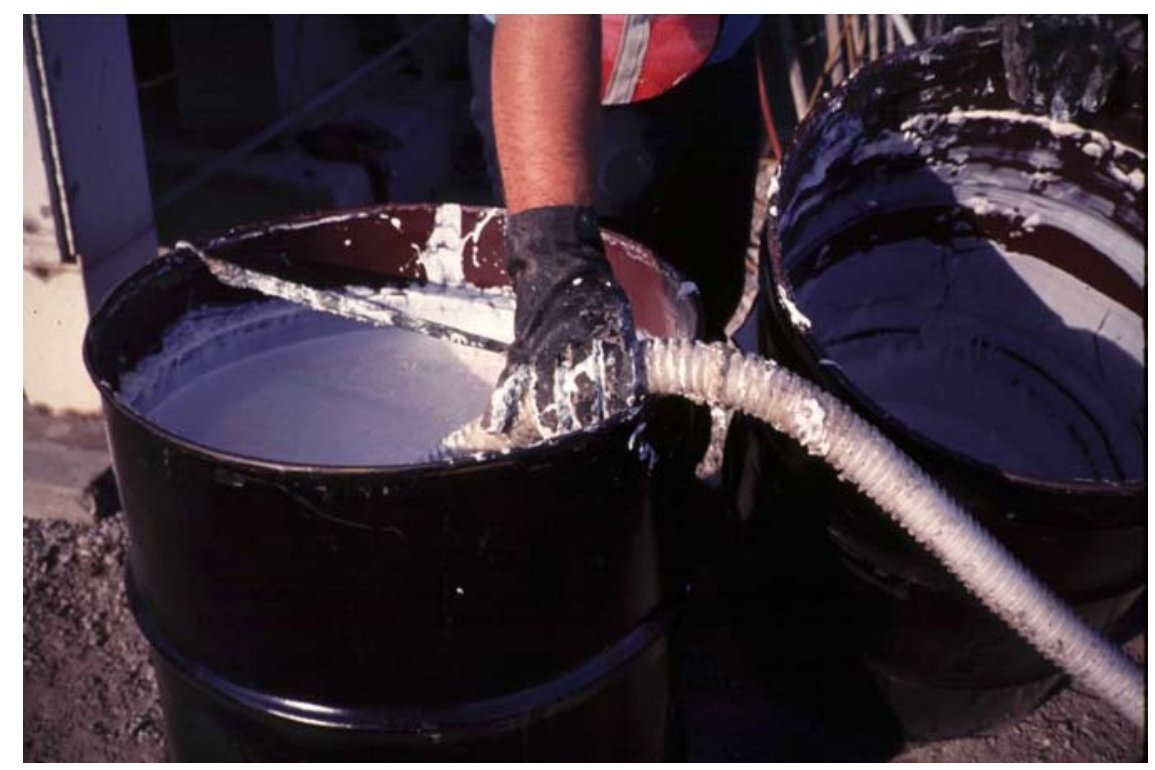

Figure 11 Pumping SBR Latex from Barrel into Plant 


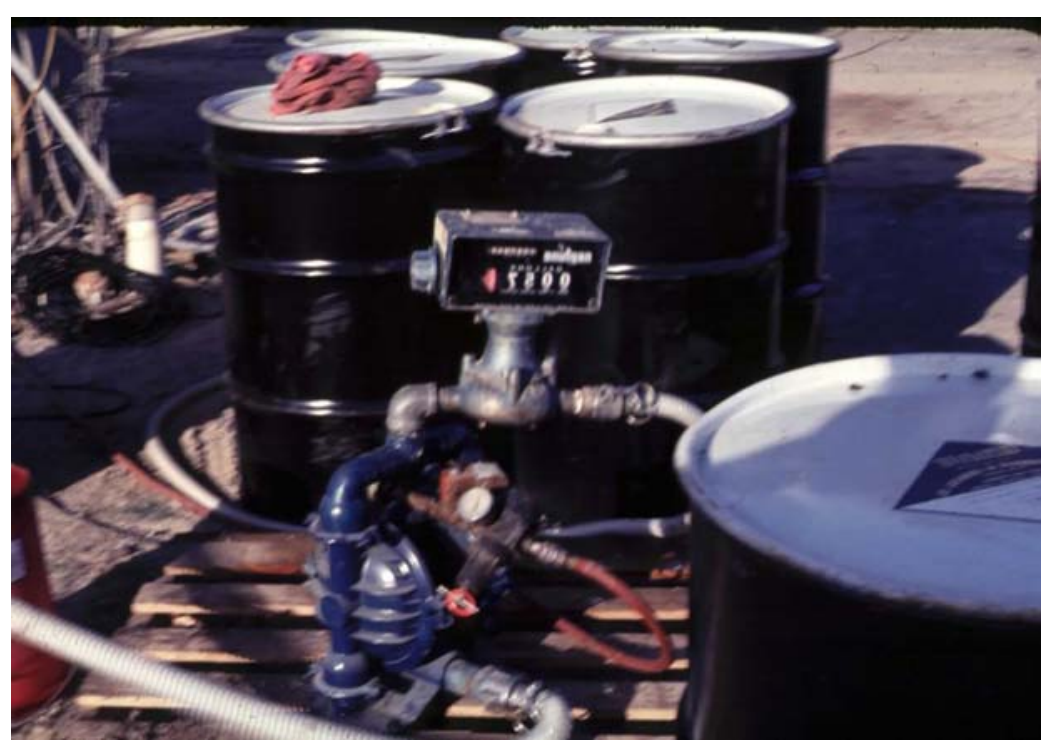

Figure 12 Meter Used to Control Flow of SBR into Plant

The SBR mixes were produced and placed on August $6^{\text {th }}$, August $7^{\text {th }}$ and September $10^{\text {th }}$. Placement and compaction were routine.

\subsubsection{Neoprene}

The neoprene mixes were produced August $8^{\text {th }}$, August $9^{\text {th }}$ and September $11^{\text {th }}$. No problems were noted on any of these days. Neoprene latex was supplied in a tanker and metered into the normal AC line at the plant (Figure 8).

\subsubsection{Asphalt Rubber}

The asphalt rubber required special mixing prior to incorporation in the mix. The mixing unit shown in Figure 13 was provided by Asphalt Rubber Systems. A tanker was used to hold the blended binder after mixing to allow the $\mathrm{AC}-10$ asphalt cement to react with the rubber. Bags of rubber were opened (Figure 14) and added through a hopper into the mixing unit. The rubber was then mixed with hot asphalt cement and the 5,500 gal. batch was transferred to the holding tank for reaction. $(\underline{67})$ 


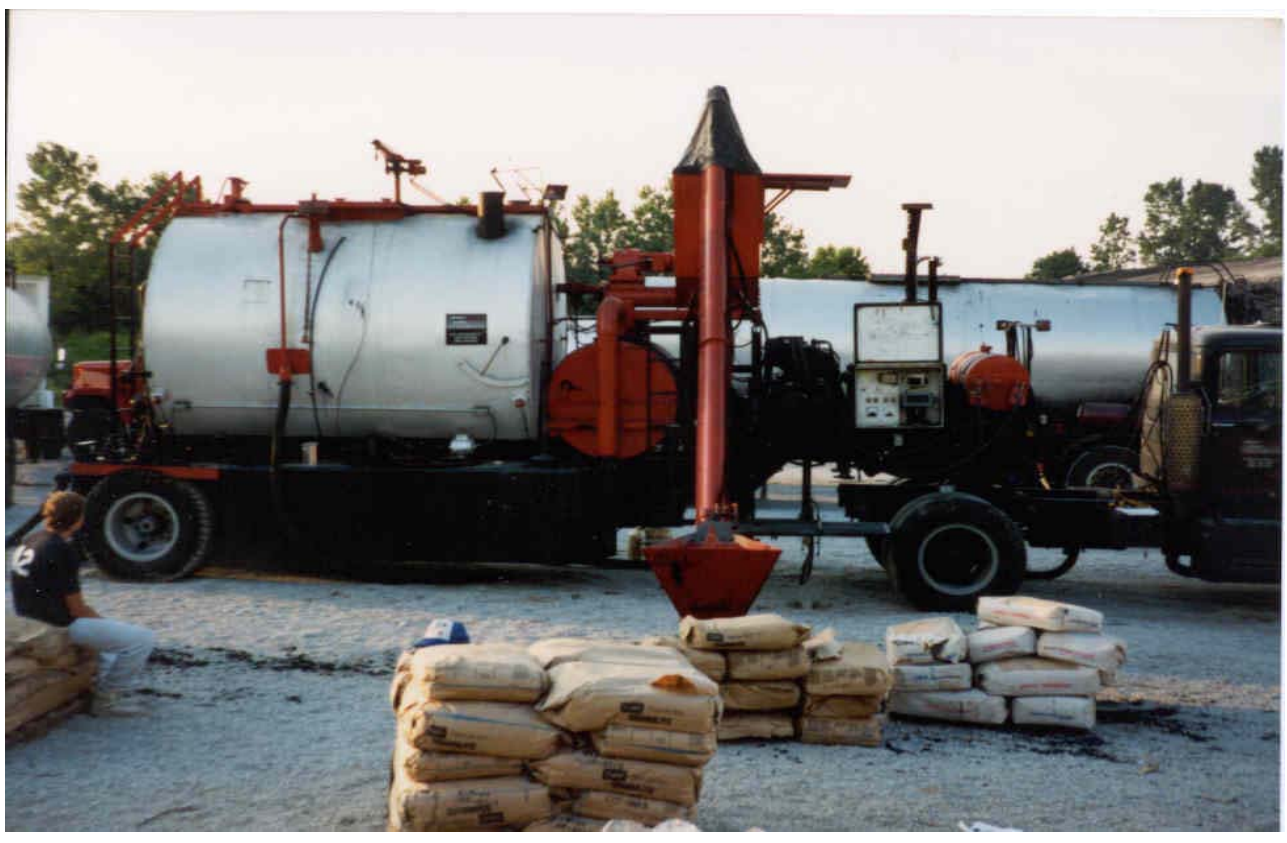

Figure 13 Asphalt Rubber Mixing Unit

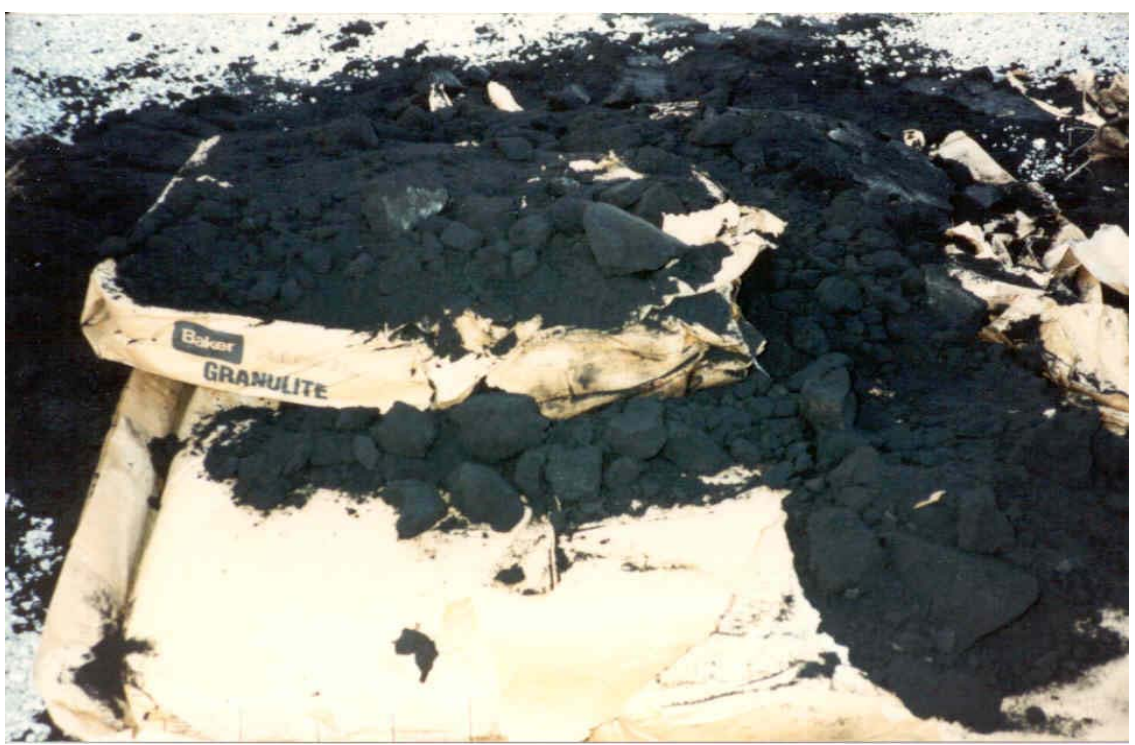

Figure 14 Ground Tire Rubber Used in Asphalt Rubber Binder

Two problems were noted with this process. First, the asphalt cement temperature needed to be quite high, as high as $190^{\circ} \mathrm{C}\left(375^{\circ} \mathrm{F}\right)$. Suppliers in Indiana generally do not store their materials at that high a temperature. When the AC-10 base asphalt arrived at the plant, it 
had to be heated in the blending unit before the rubber could be added. Second, a 45-60 minute reaction time was needed after mixing to allow the asphalt binder and rubber to react. (See Figure 15.) (The asphalt causes the rubber particles to swell but the two components do not chemically bond.) Both of these problems severely impacted production by slowing down the operations considerably. The blended and reacted binder was then hauled to the plant from a remote corner of the yard. The binder was pumped into the plant through a separate metering system provided by the supplier (Figure 16).

This process was the most labor and equipment intensive modification technique used on this project. The supplier arrived on scene with five vehicles and approximately ten people.

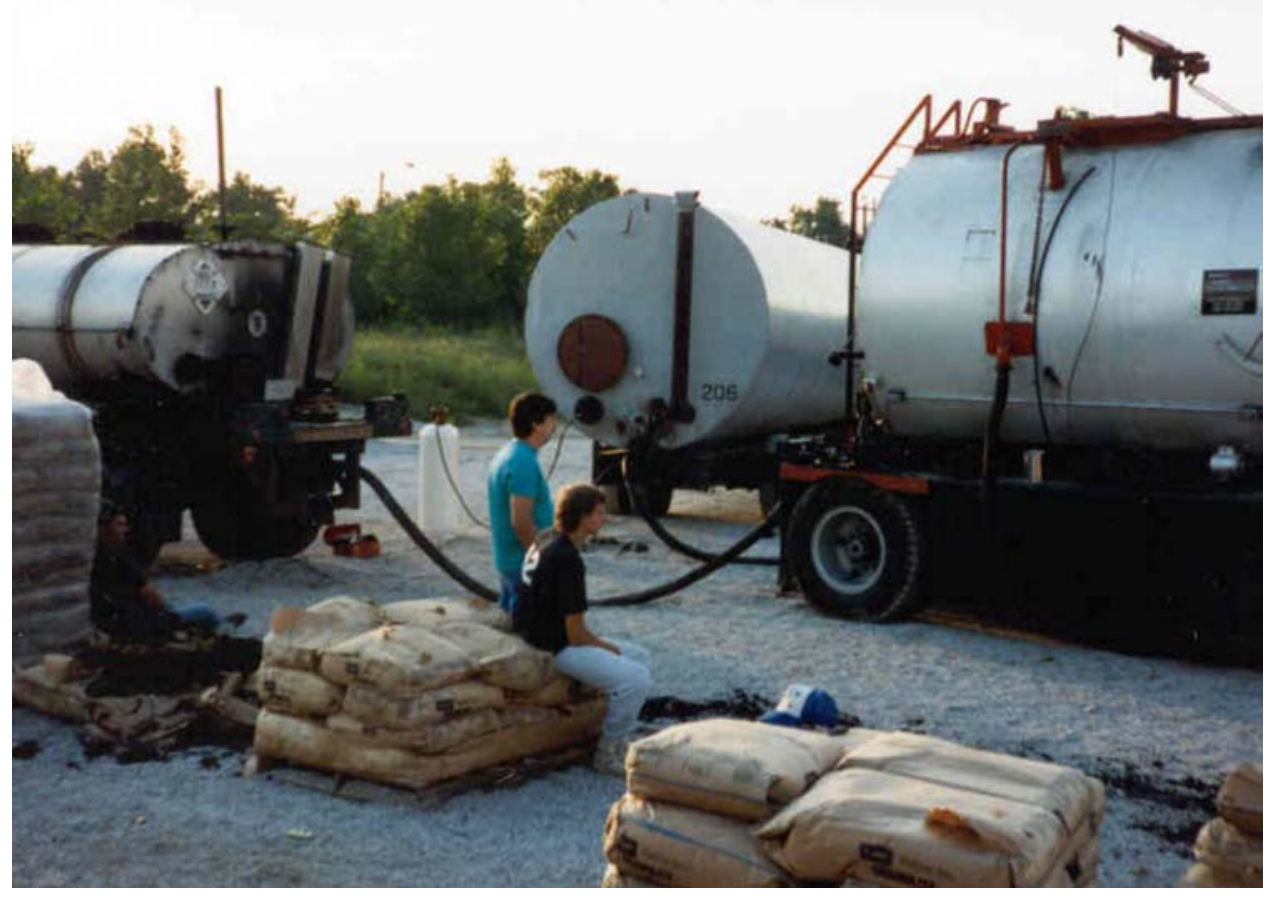

Figure 15 Waiting for the Rubber to React 


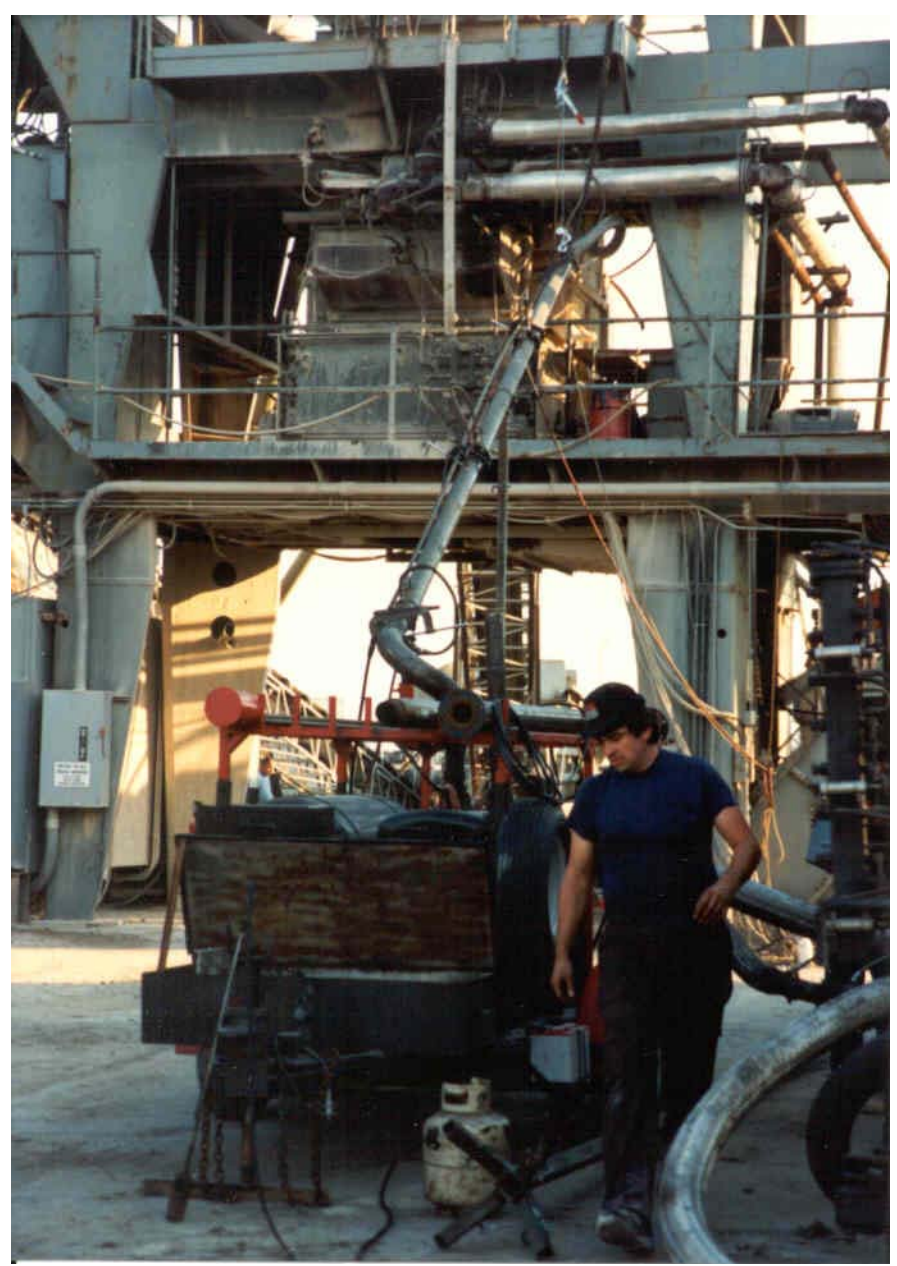

Figure 16 Pumping Reacted Asphalt Rubber into Plant

Asphalt rubber mixes were placed on August $10^{\text {th }}$ and $13^{\text {th }}$. Due to the slow production, the last 160 tons of intermediate course had to be finished on September $11^{\text {th }}$ and the surface was placed September $12^{\text {th }}$.

\subsection{Costs}

Modified binders can be significantly more expensive than unmodified binders. Although a detailed life cycle cost analysis was not a part of this project, a comparison of the relative costs of the materials is appropriate. 
Table 13 below shows the reported material and plant costs in the second through fourth columns (provided by Asphalt Materials, Inc. of Indianapolis) and the contract bid price in the fifth through seventh columns.

Table 13 Material Costs and Bid Prices

\begin{tabular}{|l|c|c|c|c|c|c|}
\hline \multirow{2}{*}{ Modifier } & \multicolumn{3}{|c|}{ Material Costs* } & \multicolumn{3}{c|}{ Bid Prices** } \\
& \multicolumn{2}{|c|}{$(\%$ increase over control) } & \multicolumn{2}{c|}{$(\%$ increase over control) } \\
\cline { 2 - 7 } & Base & Intermediate & Surface & Base & Intermediate & Surface \\
\hline Control & $\$ 13.12$ & $\$ 13.16$ & $\$ 17.23$ & $\$ 22.24$ & $\$ 23.39$ & $\$ 30.21$ \\
& $(0)$ & $(0)$ & $(0)$ & $(0)$ & $(0)$ & $(0)$ \\
\hline PAC & $\$ 16.68$ & $\$ 17.44$ & $\$ 24.22$ & $\$ 26.91$ & $\$ 29.31$ & $\$ 37.98$ \\
& $(27 \%)$ & $(32 \%)$ & $(40 \%)$ & $(21 \%)$ & $(25 \%)$ & $(62 \%)$ \\
\hline Fibers & $\$ 18.13$ & $\$ 18.81$ & $\$ 27.05$ & $\$ 33.61$ & $\$ 35.47$ & $\$ 47.01$ \\
& $(38 \%)$ & $(43 \%)$ & $(57 \%)$ & $(51 \%)$ & $(52 \%)$ & $(101 \%)$ \\
\hline PE & $\$ 18.57$ & $\$ 19.38$ & $\$ 24.57$ & $\$ 30.86$ & $\$ 33.85$ & $\$ 42.87$ \\
& $(42 \%)$ & $(47 \%)$ & $(43 \%)$ & $(39 \%)$ & $(45 \%)$ & $(83 \%)$ \\
\hline MGAC & $\$ 14.33$ & $\$ 15.98$ & $\$ 21.71$ & $\$ 23.85$ & $\$ 26.77$ & $\$ 33.05$ \\
& $(9 \%)$ & $(21 \%)$ & $(26 \%)$ & $(\%)$ & $(14 \%)$ & $(41 \%)$ \\
\hline SBR & $\$ 15.50$ & $\$ 16.66$ & $\$ 21.39$ & $\$ 29.01$ & $\$ 31.80$ & $\$ 38.49$ \\
& $(18 \%)$ & $(26 \%)$ & $(24 \%)$ & $(30 \%)$ & $(36 \%)$ & $(64 \%)$ \\
\hline Neoprene & $\$ 17.44$ & $\$ 18.58$ & $\$ 23.84$ & $\$ 29.45$ & $\$ 32.28$ & $\$ 40.84$ \\
& $(33 \%)$ & $(41 \%)$ & $(38 \%)$ & $(32 \%)$ & $(38 \%)$ & $(75 \%)$ \\
\hline AR & $\$ 36.14$ & $\$ 37.19$ & $\$ 42.17$ & $\$ 29.24$ & $\$ 30.42$ & $\$ 35.67$ \\
& $(175 \%)$ & $(182 \%)$ & $(145 \%)$ & $(31 \%)$ & $(30 \%)$ & $(52 \%)$ \\
\hline
\end{tabular}

*Material and plant costs only, exclusive of labor and laydown costs.

**Price per ton of mix in place.

Three things are obvious from reviewing this table. First, all of the modifiers are indeed more expensive than the unmodified AC-20 control, as expected. Second, some of the modifiers are much more expensive than others. The asphalt rubber is the most expensive modifier by far, due at least in part to the heavy labor and equipment requirements noted before. This leads to the third point; the reported material and plant costs for the asphalt rubber exceed the bid prices. Due to an apparent miscommunication between the asphalt rubber supplier and the contractor, the contractor's bid price was not accurate. Resolution of this dispute required the assistance of the 
legal profession. The material costs reported are more representative of the percent cost increase due to using asphalt rubber than the bid prices would suggest.

Looking at the material costs, then, most of the modifiers increased the costs by roughly 10 to $40 \%$. The asphalt rubber, however, cost two to three times as much as the control mixtures. The rubber would have to perform extremely well in order to justify this expense. Field performance will be discussed in detail in chapter 5 .

Modification is more common now than it was in 1990, so these increases may no longer be representative. Ecomony of scale may also help to reduce the costs. The quantities of materials used on this project were quite small. 


\section{FINDINGS FROM FIELD TRIAL}

One of the major features of this project is the tie between laboratory and field performance. This section describes the field performance of the test and control sections since construction. Deflection testing is discussed first in section 4.1. Visual distress surveys, which provide the most revealing data about the observed field performance, are described in section 4.2. Friction testing results are summarized in section 4.3, and condition data from INDOT's roadway management program is outlined in section 4.4 .

\subsection{Deflection Testing}

The Dynaflect was used to measure the response of the pavement to load before and after construction. The Dynaflect is a trailer-mounted device that applies a cyclic 1,000 pound load to the pavement through two steel wheels. The frequency of loading is 8 cycles per second. The deflection of the pavement under this load is measured by five geophones spaced at one foot intervals from between the wheels out four feet in advance of the load. The geophones measure small deflections through the use of a coil spring mounted within a magnet. Changes in the voltage brought about by the movement of the coil relative to the magnet can be converted to deflection measurements.

The deflection basin produced by the cyclic loading can be analyzed in several ways to determine various properties of the pavement system, including the subgrade. Majidzadeh and Kumar summarized the deflection parameters and their significance in 1983. According to them, the magnitude of the deflection under the steel wheels (W1) is an indicator of the relative strength of the road section. The Surface Curvature Index (SCI) and Spreadability (SPR) indicate the relative strength of the upper bound layers. The Base Curvature Index (BCI) and value of the deflection farthest from the load (W5) are indicators of the base and subgrade strength. The SCI was commonly used to measure load transfer across joints in Portland cement concrete pavements. (마)

INDOT had a long and successful history of using the Dynaflect to determine where pavement support was lacking and undersealing was needed to improve the support. Majidzadeh and Kumar's parameters were commonly used in research to assess the strengths of various 
pavement layers. In the early 1990's, INDOT began to switch from use of the Dynaflect to the Falling Weight Deflectometer, which was felt to provide better information about the pavement structure for pavement design. Dynaflects are now no longer used by INDOT, having been replaced by Falling Weight Deflectometers.

Dynaflect testing was performed before undersealing to determine the appropriate locations for this maintenance treatment. Hot liquid asphalt is pumped under pressure under the pavement to fill any voids and improve the support conditions. Testing was also conducted after overlaying in November 1990 to verify that the pavement and underlying support conditions were uniform. The data from the post-construction testing on the different sections is summarized in Table 14. This data shows that the sections are quite uniform. Though a statistical analysis was not conducted on this data, the averages and ranges are quite clearly nearly identical.

In addition, comparison of these test results to Majidzadeh and Kumar's parameters shows that the subgrade and pavement layers are in good to excellent conditions. Majidzadeh and Kumar suggest W1 values should be no higher than 0.40 to 0.50 mils for rigid pavements and no higher than 0.70 to 1.0 mils for flexible pavements. Most of the values for these overlaid test sections are less than 0.32 and all are less than 0.42 . These values show that the upper bound layers of the pavement (concrete and asphalt) are in good to excellent condition.

W5 values of less than or equal to 0.15 indicate good base and subgrade conditions, and values greater than or equal to 0.30 mils are considered poor. Again, nearly all of the values are in the good category, with only a few readings in the gray area. All of the values are less than 0.20 . BCI values also indicate the subgrade and base conditions. Values of greater than 0.11 mils suggest there may be problems, while lower values are acceptable. None of the BCI values for any of the test sections were greater than 0.07 . 
Table 14 Summary of Dynaflect Parameters

\begin{tabular}{|c|c|c|c|c|c|}
\hline Section & $\begin{array}{c}\text { Average W1 } \\
(\text { Range })\end{array}$ & $\begin{array}{c}\text { Average W5 } \\
(\text { Range })\end{array}$ & $\begin{array}{c}\text { Average SCI } \\
(\text { Range })\end{array}$ & $\begin{array}{c}\text { Average BCI } \\
\text { (Range) }\end{array}$ & $\begin{array}{c}\text { Average SPR } \\
(\text { Range })\end{array}$ \\
\hline $\begin{array}{c}\text { A. Control } \\
\text { (SB) }\end{array}$ & $\begin{array}{c}0.25 \\
(0.20-0.42)\end{array}$ & $\begin{array}{c}0.14 \\
(0.09-0.20)\end{array}$ & $\begin{array}{c}0.04 \\
(0.02-0.06)\end{array}$ & $\begin{array}{c}0.03 \\
(0.00-0.07)\end{array}$ & $\begin{array}{c}77.8 \% \\
(60.7-85.2)\end{array}$ \\
\hline B. PAC & 0.22 & 0.12 & 0.03 & 0.03 & $76.7 \%$ \\
& $(0.14-0.33)$ & $(0.07-0.17)$ & $(0.02-0.04)$ & $(0.02-0.04)$ & $70-84.2)$ \\
\hline C. Fiber & 0.24 & 0.14 & 0.03 & 0.03 & $78.8 \%$ \\
& $(0.19-0.34)$ & $(0.09-0.18)$ & $(0.02-0.06)$ & $(0.02-0.04)$ & $(72-83)$ \\
\hline D. PE & 0.25 & 0.14 & 0.03 & 0.03 & $79.8 \%$ \\
& $(0.19-0.34)$ & $(0.09-0.18)$ & $(0.00-0.06)$ & $(0.02-0.04)$ & $(72-83)$ \\
\hline E. MGAC & 0.24 & 0.13 & 0.03 & 0.03 & $74.5 \%$ \\
& $(0.17-0.34)$ & $(0.08-0.19)$ & $(0.00-0.07)$ & $(0.02-0.04)$ & $(65-82.6)$ \\
\hline F. SBR & 0.20 & 0.10 & 0.02 & 0.02 & $75.7 \%$ \\
& $(0.17-0.25)$ & $(0.01-0.14)$ & $(0.00-0.05)$ & $(0.02-0.03)$ & $(67.8-83.6)$ \\
\hline G. Neo & 0.16 & 0.08 & 0.03 & 0.02 & $75.6 \%$ \\
& $(0.09-0.23)$ & $(0.05-0.12)$ & $(0.01-0.05)$ & $(0.01-0.04)$ & $(69.4-81.3)$ \\
\hline H. AR & 0.20 & 0.10 & 0.03 & 0.03 & $76.4 \%$ \\
& $(0.15-0.25)$ & $(0.07-0.14)$ & $(0.01-0.06)$ & $(0.02-0.04)$ & $(65.6-83.5)$ \\
\hline J. Control & 0.22 & 0.12 & 0.03 & 0.03 & $79.2 \%$ \\
(NB) & $(0.16-0.31)$ & $(0.05-0.20)$ & $(0.01-0.06)$ & $(0.01-0.04)$ & $(68.2-86.7)$ \\
\hline
\end{tabular}

The last parameter, spreadability, is usually used as an indication of load transfer efficiency across joints or cracks, but can also indicate general pavement conditions. SPR decreases when the pavement is cracked or in poor condition. Values greater than $75 \%$ for rigid and greater than $60 \%$ for flexible pavements are considered satisfactory. All of the average SPR values are greater than $75 \%$, as are most of the individual readings. None of the individual determinations fall below $60 \%$.

This data demonstrates that the pavement was in good to excellent condition shortly after construction and the conditions were uniform throughout. The readings show that the concrete pavement and the base and subgrade conditions were all acceptable. The care that was taken to patch any deteriorated areas of the concrete was apparently successful in limiting structural problems. No signs of structural distresses were observed during any of the field investigations since the time of construction. 


\subsection{Visual Distress Surveys}

Initially, a detailed visual distress survey was conducted on the entire length of the project. This was very time-consuming, even though the detailed examination was confined to the high speed lane, due to traffic control considerations. (The presence of two ramps within the project boundaries made it unsafe to close the center or right-hand lanes.) The subsequent detailed distress surveys were conducted on only a randomly selected $305 \mathrm{~m}$ (1000 foot) section. A comparison of the development of cracks in the test sections, then, is presented here for the $305 \mathrm{~m}$ (1000 foot) section.

The $305 \mathrm{~m}$ (1000 foot) survey section was selected by using a computer program to generate random numbers. When a random number fell within the station limits for a test section, it was selected as the starting point for the detailed survey. Numbers falling in the first $152 \mathrm{~m}$ (500 feet) of a test section (in the direction of paving) were discarded to avoid areas where there may have been start-up problems at the plant during construction.

Detailed surveys were conducted in 1993, 1996 and 2001. During these inspections, rut depth was measured at every even station in the left and right wheelpaths with a $1.2 \mathrm{~m}$ (4-foot) straightedge. Cracks were mapped and sketched in the field. The location of the cracks was determined using a rolling wheel. The results of the visual inspections are summarized in Tables 15 and 16 and Figures 17 through 25 . In the figures, "L" is the sum of the longitudinal cracking and " $\mathrm{T}$ " is the sum of the transverse cracking in each $305 \mathrm{~m}(1000 \mathrm{ft}$.) section.

Table 15 I-465 Pavement Condition Surveys - Cracking Summary

\begin{tabular}{|c|c|c|c|c|c|c|}
\hline \multirow{2}{*}{ Section } & \multicolumn{3}{|c|}{ Transverse Cracking, Sum (lft) } & \multicolumn{2}{c|}{ Longitudinal Cracking, Sum (lft) } \\
\cline { 2 - 7 } & 1993 & 1996 & 2001 & 1993 & 1996 & 2001 \\
\hline A- Control & 174 & 416 & NA & 142 & 507 & NA \\
\hline B- PAC & 24 & 87 & 186 & 15 & 198 & 665 \\
\hline C- Fiber & 144 & 150 & 178 & 30 & 70 & 370 \\
\hline D- PE & 78 & 222 & $>696$ & 615 & 1485 & $>2500$ \\
\hline E- MGAC & 150 & 231 & 468 & 75 & 220 & 390 \\
\hline F- SBR & 12 & 62 & 156 & 0 & 0 & 260 \\
\hline G- Neo & 123 & 237 & 320 & 0 & 6 & 340 \\
\hline H- AR & 96 & 156 & 178 & 135 & 155 & 300 \\
\hline J- Control & 182 & 261 & 580 & 500 & 939 & 1400 \\
\hline
\end{tabular}

$\mathrm{NA}=$ Not Available, Section Overlaid 

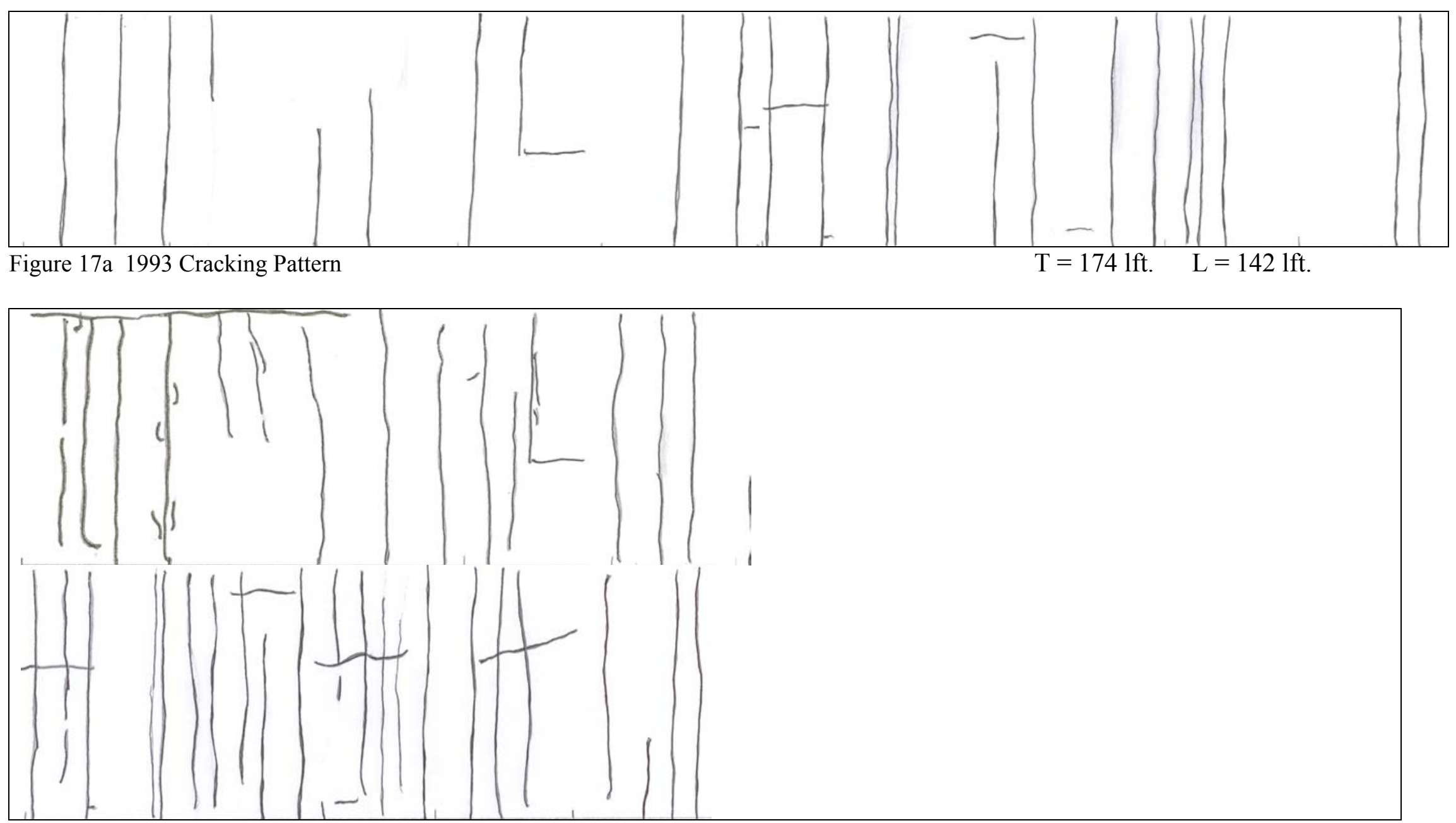

Figure 17b 1996 Cracking Pattern

$\mathrm{T}=4161 \mathrm{ft}$.

$\mathrm{L}=507 \mathrm{lft}$ 
Figure 17 Development of Cracking in Control Section (A)
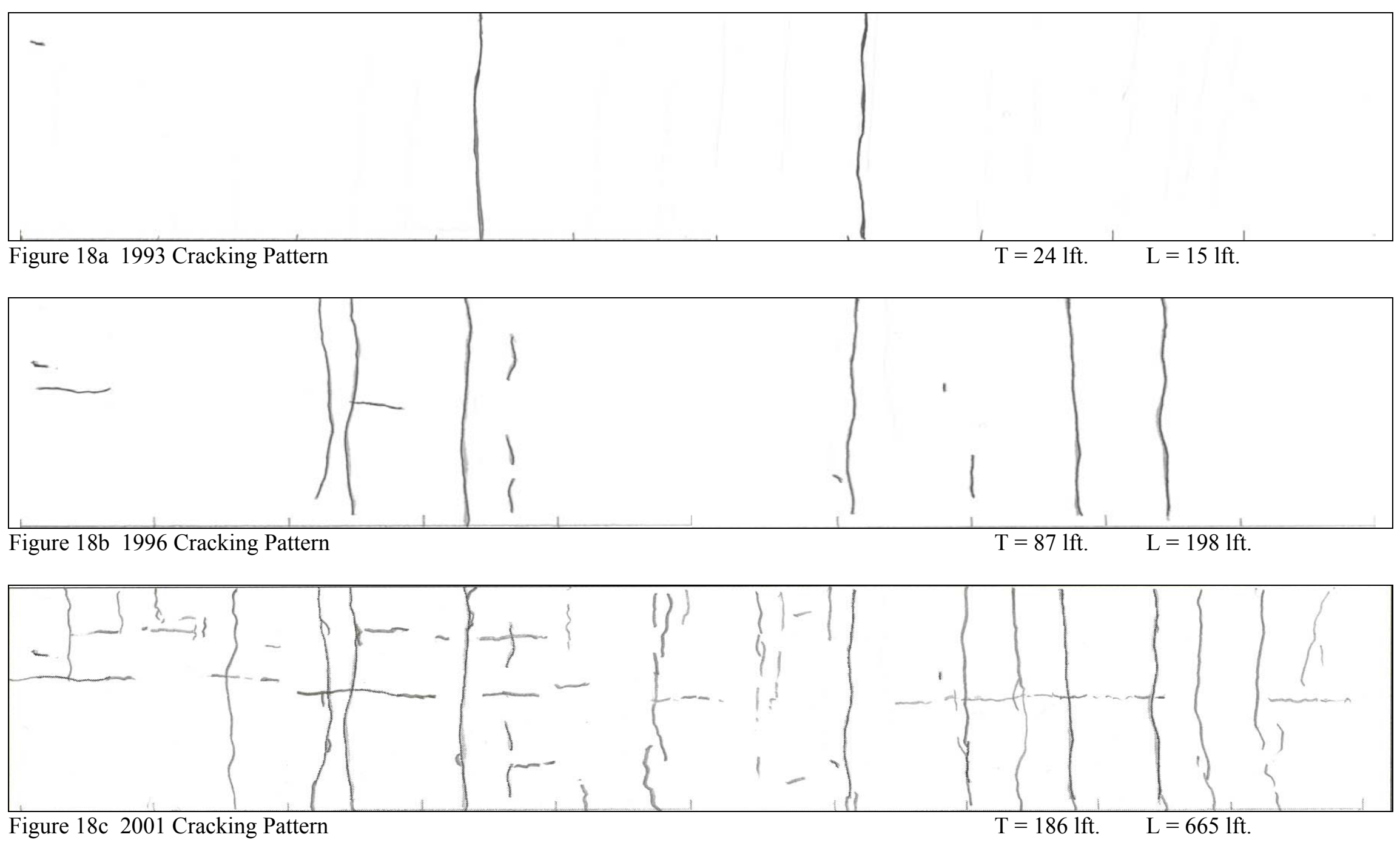
Figure 18 Development of Cracking in PAC Section (B)
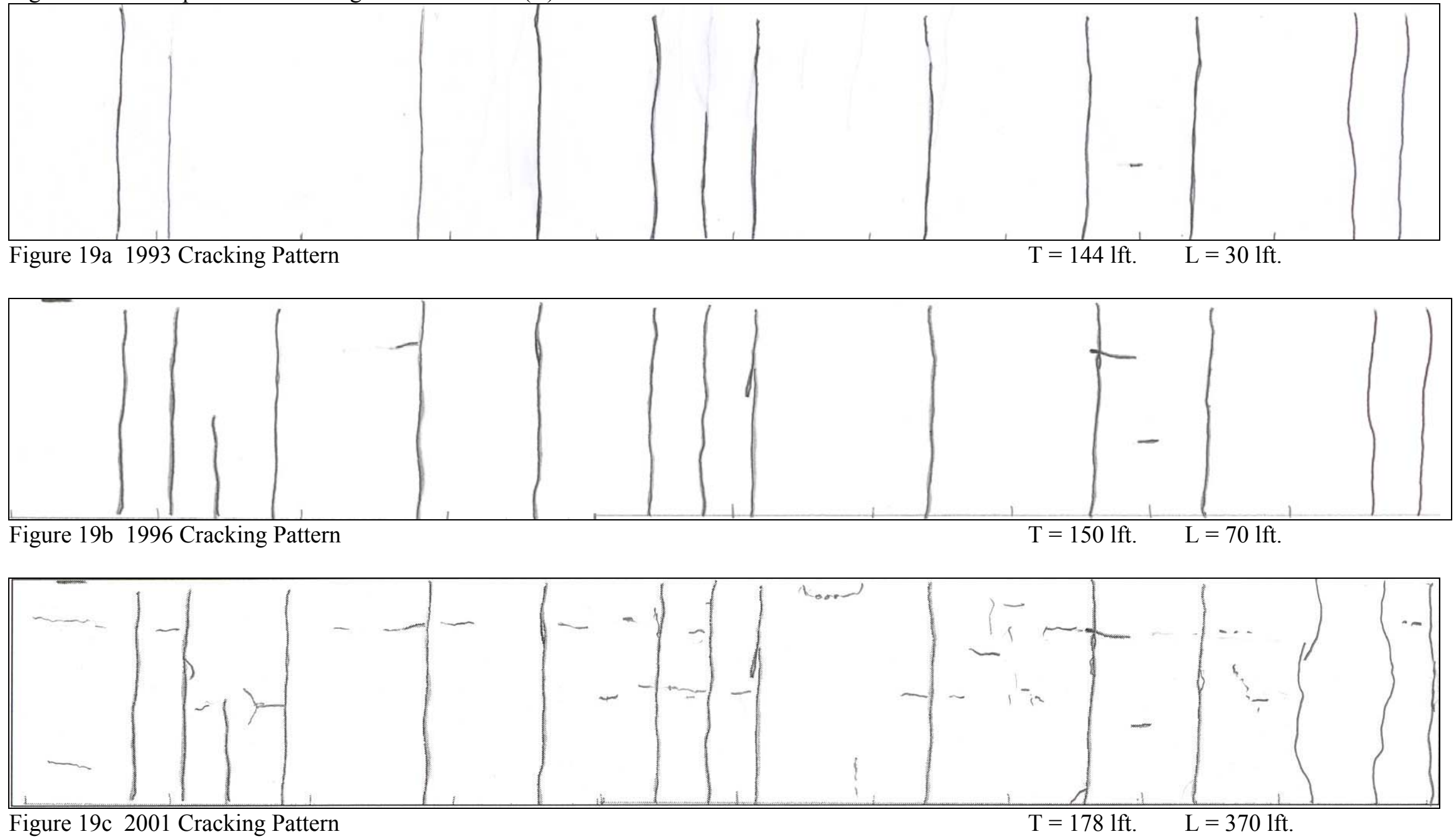

Figure 19 Development of Cracking in Fiber Section (C) 

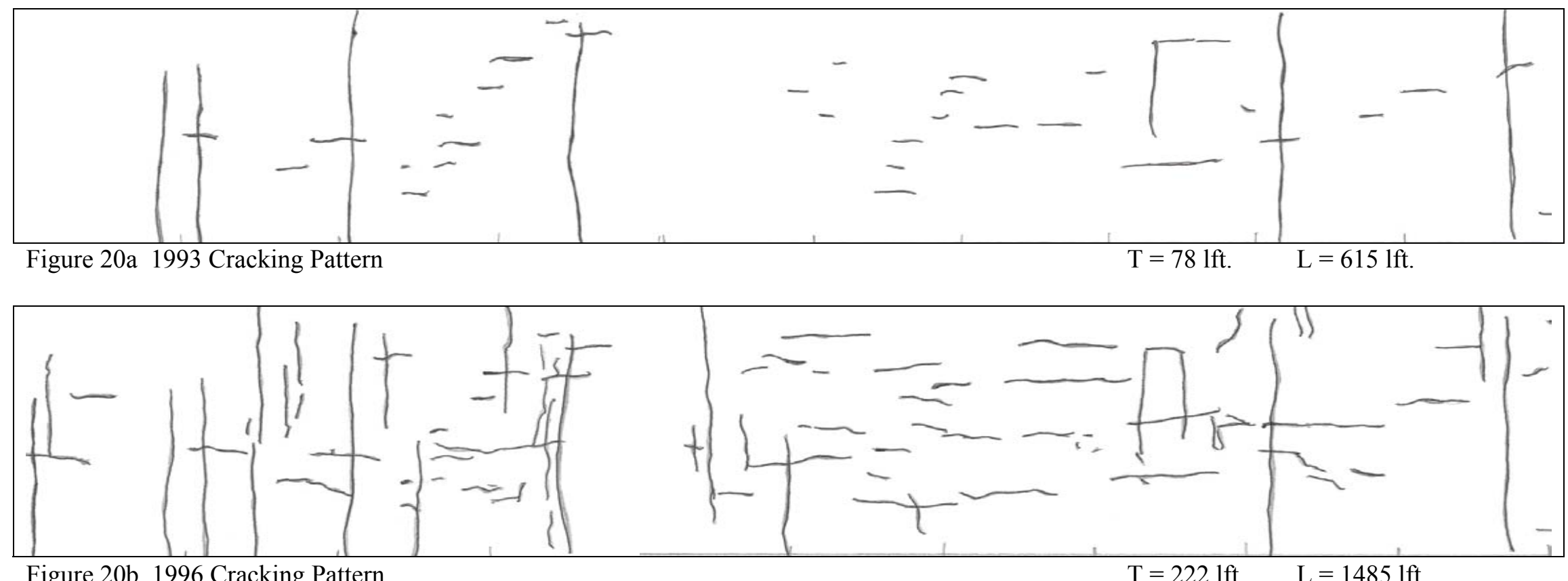

Figure 20b 1996 Cracking Pattern

$\mathrm{T}=222 \mathrm{lft}$

$=1485 \mathrm{lft}$.

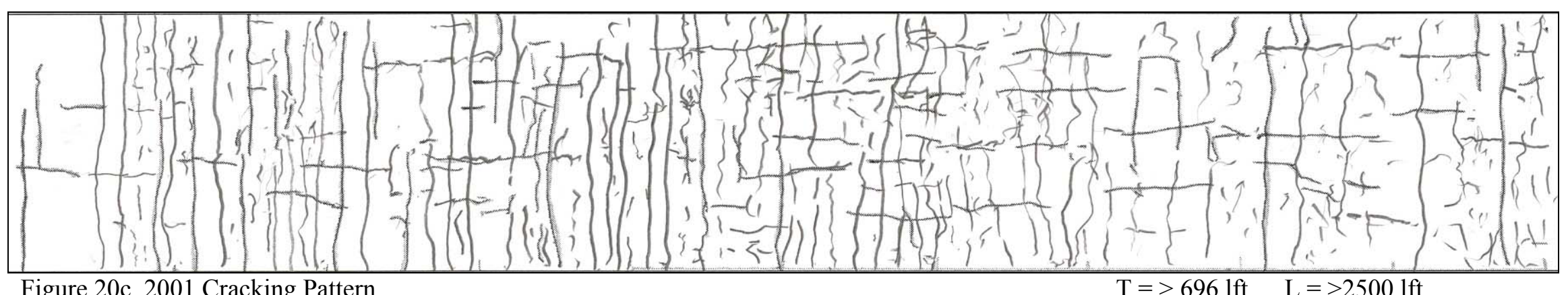

Figure 20 Development of Cracking in PE Section (D) 


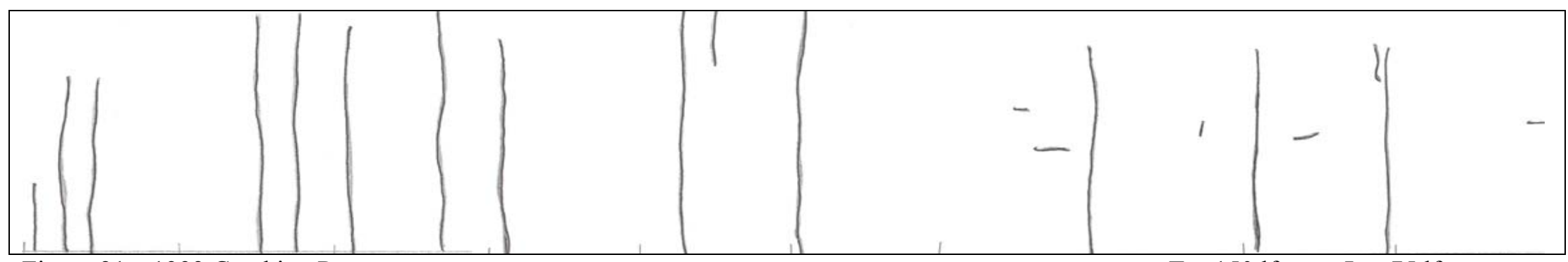

Figure 21a 1993 Cracking Pattern

$\mathrm{T}=150 \mathrm{lft}$

$\mathrm{L}=75 \mathrm{lft}$.

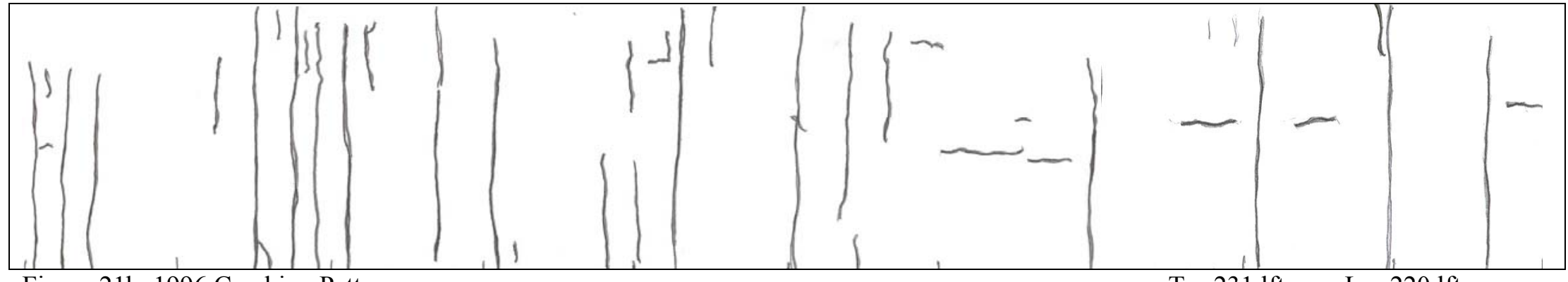

Figure 21b 1996 Cracking Pattern

$\mathrm{T}=2311 \mathrm{ft} . \quad \mathrm{L}=220 \mathrm{lft}$.

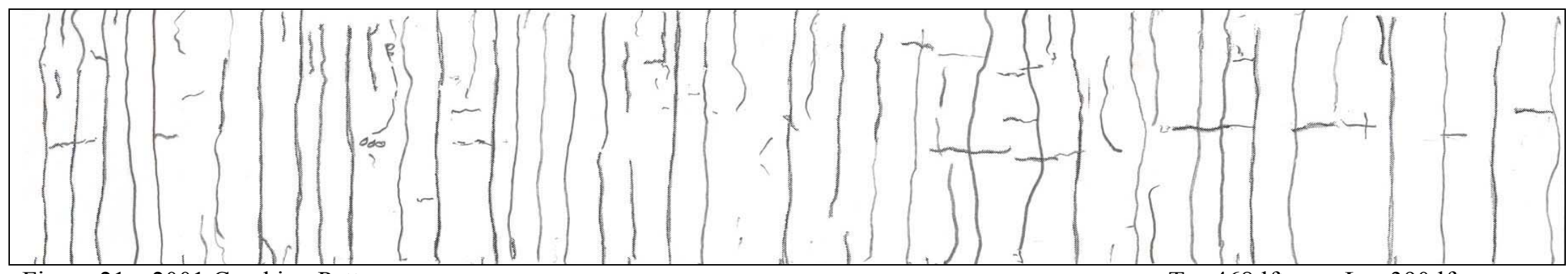

Figure 21c 2001 Cracking Pattern

$\mathrm{T}=468 \mathrm{lft}$.

$\mathrm{L}=390 \mathrm{lft}$.

Figure 21 Development of Cracking in MGAC Section (E) 


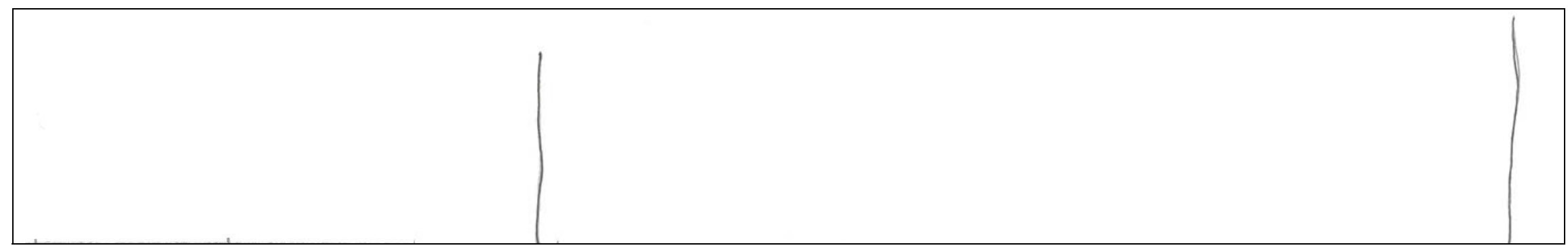

Figure 22a 1993 Cracking Pattern

$\mathrm{T}=12 \mathrm{lft}$.

$\mathrm{L}=0 \mathrm{lft}$.
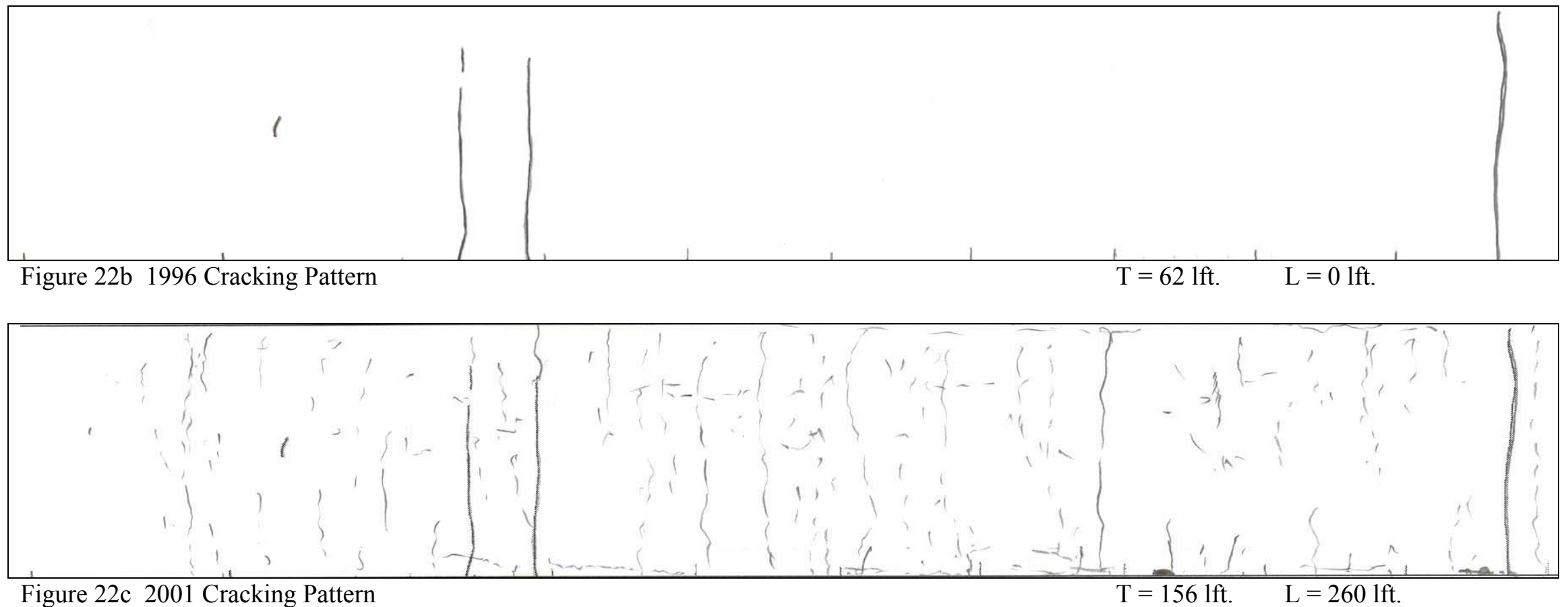

Figure 22 Development of Cracking in SBR Section (F) 


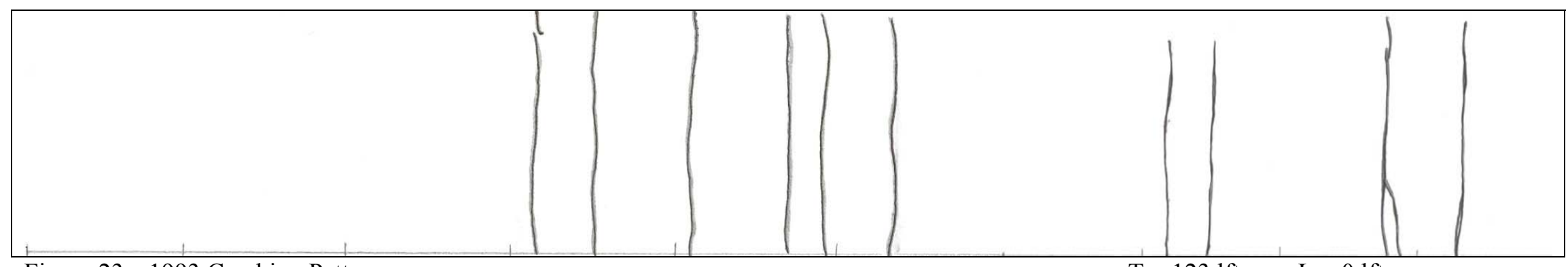

Figure 23a 1993 Cracking Pattern

$\mathrm{T}=123 \mathrm{lft} . \quad \mathrm{L}=0 \mathrm{lft}$.

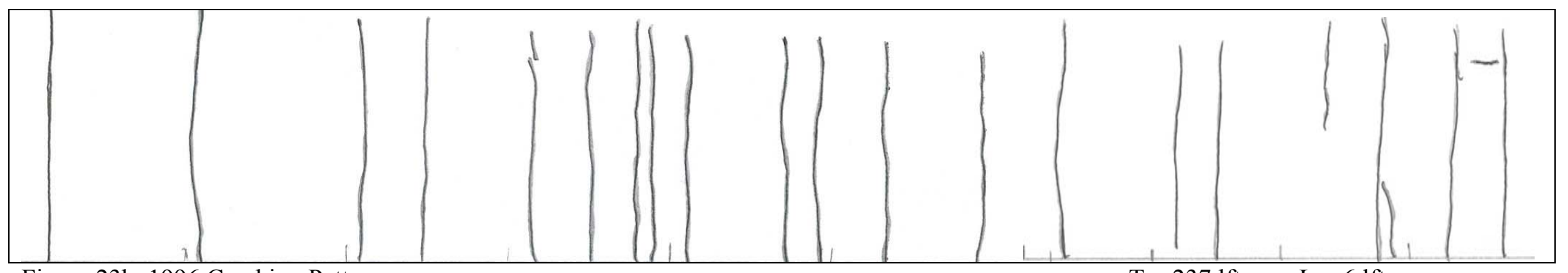

Figure 23b 1996 Cracking Pattern

$\mathrm{T}=237$ lft. $\quad \mathrm{L}=6 \mathrm{lft}$.

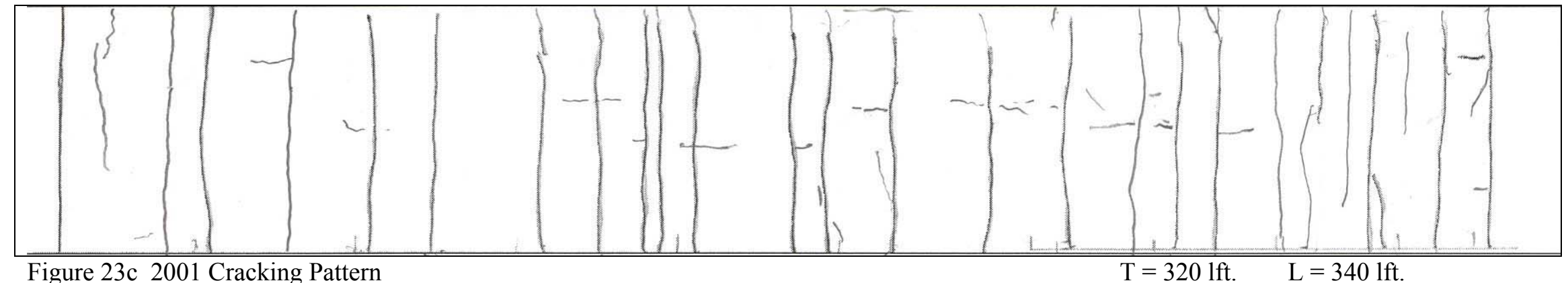

Figure 23 Development of Cracking in Neo Section (G) 


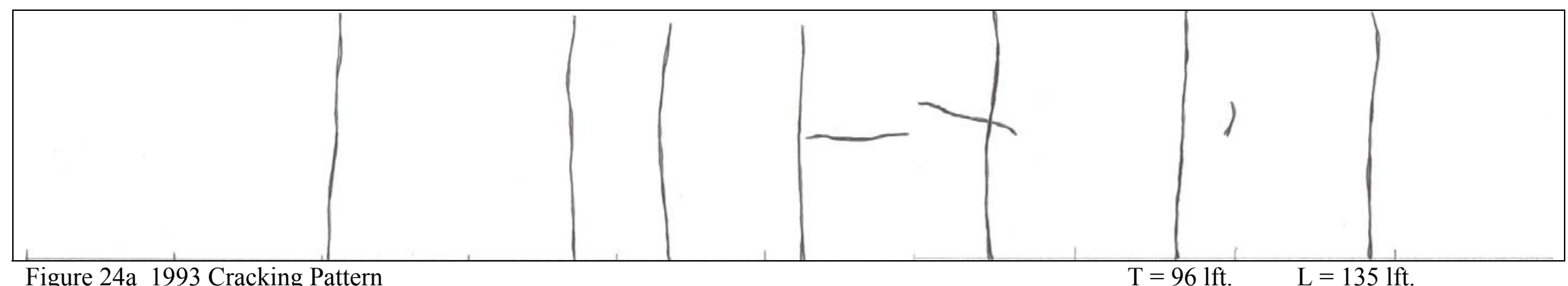

Figure 24a 1993 Cracking Pattern

$\mathrm{T}=96 \mathrm{lft} . \quad \mathrm{L}=135 \mathrm{lft}$.
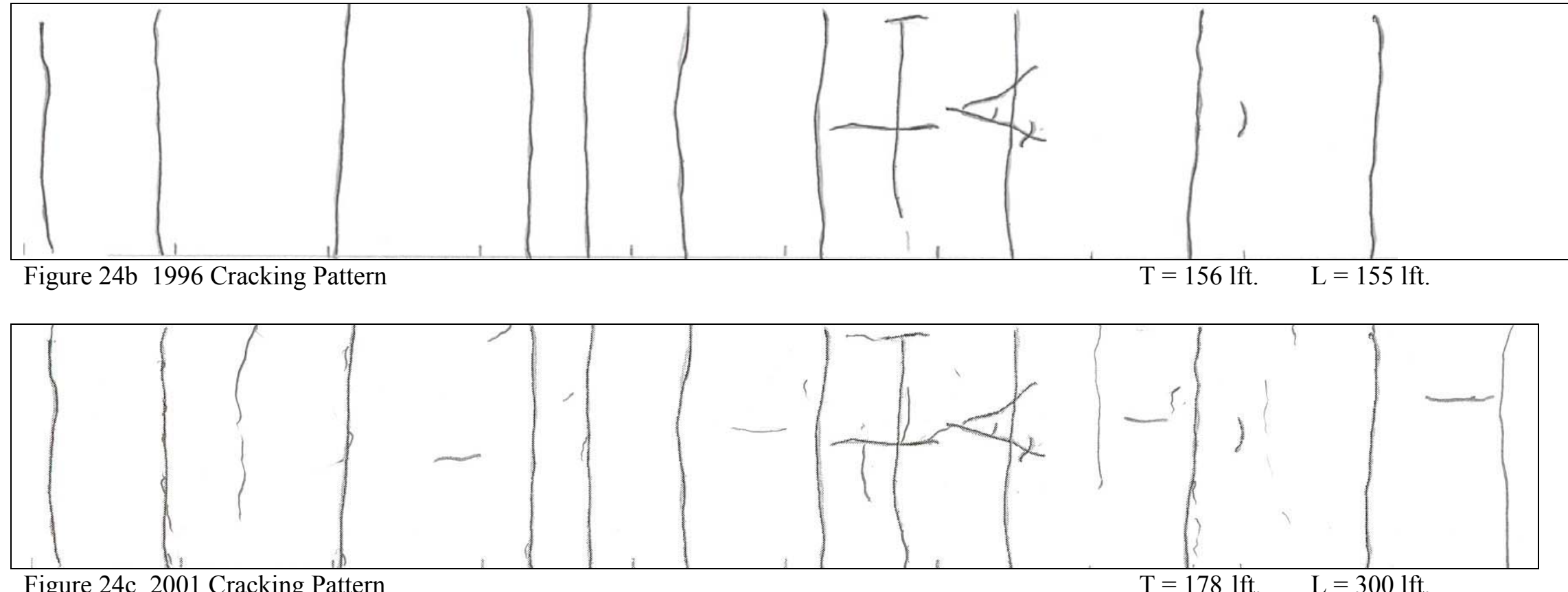

Figure 24 Development of Cracking in AR Section (H) 

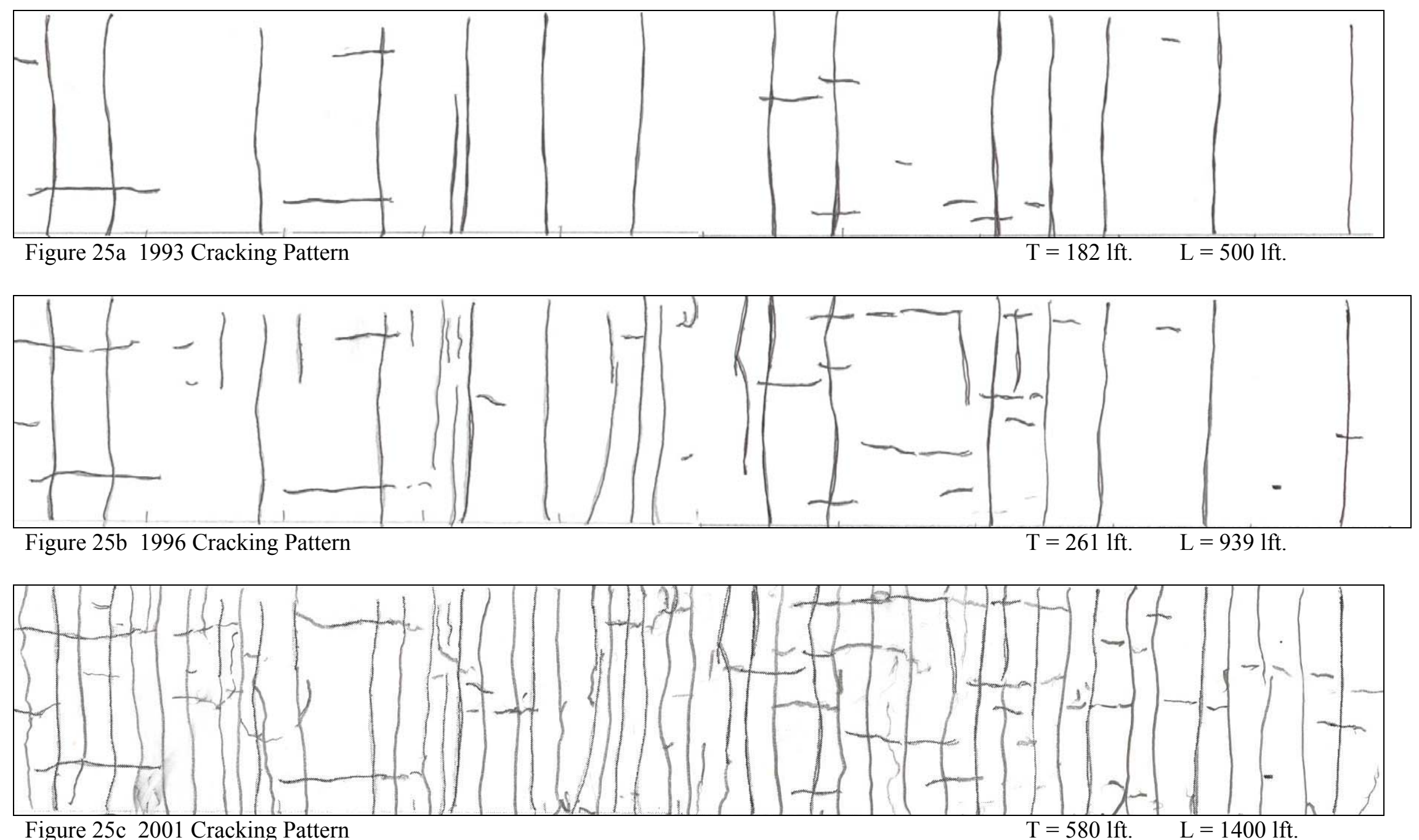

Figure 25 Development of Cracking in Control Section (J) 
Table 16 I-465 Condition Surveys - Rutting Summary

\begin{tabular}{|c|c|c|c|c|}
\hline \multirow{2}{*}{ Section } & \multicolumn{4}{|c|}{ Rut Depth in $1 / 16^{\text {th }}$ of inch } \\
\cline { 2 - 5 } & Left 1993 & Right 1993 & Left 1996 & Right 1996 \\
\hline A- Control & 1.0 & 0.8 & 0.3 & 0.9 \\
\hline B- PAC & 1.0 & 0.4 & 0.4 & 0.5 \\
\hline C & 0.8 & 0.2 & 0.2 & 0.8 \\
\hline D & 0.3 & 0.1 & 0.1 & 0.3 \\
\hline E & 0.9 & 0.5 & 0.9 & 0.9 \\
\hline F & 0.7 & 0.3 & 0.4 & 0.6 \\
\hline G & 0.8 & 0.0 & 0.2 & 0.2 \\
\hline H & 1.2 & 0.3 & 0.4 & 0.8 \\
\hline J- Control & 0.9 & 0.4 & 0.7 & 0.8 \\
\hline
\end{tabular}

NA = Not Available, Section Overlaid.

Table 15 shows the sum of the transverse and longitudinal cracking in the $305 \mathrm{~m}$ (1000 ft.) measurement sections. Figures 17 through 25 graphically illustrate the development of cracks from 1993 to 1996 to 2001 . From these figures, it is obvious that some sections have substantially more cracking than others. The two Control, Fiber, MGAC, Neoprene and Asphalt Rubber sections all developed about $30.5 \mathrm{~m}$ (100 ft.) or more of transverse cracking within three years of construction. The SBR and PAC sections had less than $7.6 \mathrm{~m}(25 \mathrm{ft}$.) of transverse cracking in the same time period.

Six years after construction, cracking had continued to develop on all of the sections, but it was progressing more rapidly in some sections than others. The Control sections, PE, MGAC and Neoprene sections had developed extensive transverse cracking, over $61.0 \mathrm{~m}$ (200 ft.) of cracking per 304.8m (1000 ft.) measuring section. The Fibers and Asphalt Rubber had around $150 \mathrm{ft}$. of transverse cracking. The SBR and PAC had developed under 30.5m (100 ft.) of transverse cracking after six years.

In 2001, after 11 years in service, cracking continued to develop in all of the sections, though again at varying rates. The northbound Control section and the PE section were by far the worst. (The southbound Control section was overlaid around 1999 during rehabilitation of an 
adjacent section.) The MGAC, SBR, Neoprene and AR sections exhibited the slowest crack development. The AR section, in fact, changed very little from 1996 to 2001.

Longitudinal cracking also developed within three years on some of the test sections, most notably the control sections and the PE and AR sections. The controls and the PE sections continued to develop longitudinal cracking over the next three years. In fact, the PE section developed extensive longitudinal cracking by 1996. No longitudinal cracking had developed on the SBR section after six years and only minimal cracking had developed on the Neoprene section (1.8m (6 ft.)).

The crack severity (width, braiding, etc.) was generally low to moderate. Maintenance on this pavement was been quite thorough. Cracks were sealed roughly every five years with an asphalt rubber crack sealant. This was last performed in 1999. Other cracks that developed were sealed conventionally roughly every year. This has apparently helped to prevent the cracks from becoming severe, even in the control sections.

The rutting values shown in Table 16 represent the average rut depth measured at each station within the $304.8 \mathrm{~m}$ (1000 ft.) measurement sections. Values vary from year to year due to slight differences in measurement location. (Values were not recorded at every station in 2001 because there was so little rutting.) No measurements greater than $1 / 16^{\text {th }}$ inch were made. The important point is that there is only about $1.6 \mathrm{~mm}\left(1 / 16^{\text {th }}\right.$ in.) of rutting or less in each section after 11 years. These measurements were taken in the high speed lane, which has less truck traffic than the other two lanes. Visual examinations and the roadway management data discussed in 5.4 , however, show that rutting is negligible in the heavily traveled lanes as well.

General observations about the condition and appearance of the test sections also reveal differences in the performance after 11 years. Many of the cracks seen on the PAC section were faint and well-sealed; few were moderately severe. Some openness of the texture was noted, but overall the section looked quite good for its age. The Fiber section also looked quite good, changing relatively little from 1996 to 2001 . The PE section was the worst by far. The entire surface was covered by a mesh of cracks from fine to moderately severe. Reflective cracks approximately every $6 \mathrm{~m}$ ( $20 \mathrm{ft}$.) could be discerned, but the transverse cracking pattern was too extensive to be purely reflective. Extensive longitudinal cracking had also developed, which was not reflective in nature.

The MGAC section appeared to have predominantly reflective cracking. The other cracking noted was relatively minor. Overall this section also looked quite good. The SBR section looked very good overall, but had a distinctly different appearance from the other 
sections. This section had disjointed, hairline to moderate cracks scattered over the pavement, rather than the linear, continuous cracks that were most common in the other sections. The SBR also seemed to have a more open surface texture and more cracking at the longitudinal joints than most of the other sections. The Neoprene and Asphalt Rubber sections looked very good and probably changed the least from 1999 to 2001 . Most of the cracking in these two sections appeared to be reflective with some longitudinal cracking. Lastly, the Control section also exhibited extensive cracking with some braiding and moderately severe cracking. The cracking was not as extensive nor as severe, however, as in the PE section.

While the cracking in some sections was quite extensive, the pavement is in remarkably good shape for its age.

\subsection{Friction Testing}

Special friction testing was performed annually on this project from 1990 through 1996 to determine if the experimental binders had any effect on the pavement friction over time. Special friction testing of this project was dropped in 1997 when INDOT changed their friction testing program from the ribbed to the smooth tire. Inventory testing has continued on the pavement since then. The frequency of testing is reduced for inventory testing, however, so specific data on the individual test sections is not available from 1997 through 2001.

Generally the binder is not recognized as contributing significantly to pavement friction. Once the initial film coating is worn off the surface, the aggregates control the frictional properties. Since the aggregates used on this project were essentially the same, no major differences in friction levels were expected. Traffic action on the aggregates can affect the friction after the binder film is worn away. As aggregates polish under traffic the friction can decrease. The binder may, however, affect the friction levels by changing the extent to which aggregate particles are held in place. If aggregate particles are dislodged by traffic, the pavement macrotexture may decrease, leading to a corresponding decline in friction.

INDOT had limited to no experience with the modified binders used on this project, however, so there was a need to verify that this past experience held true with the modified binders as well. It was conceivable that some of the binders would be more tenacious and would wear away more slowly, perhaps affecting friction for a longer period of time. Also, some of the binders might hold the aggregates in place better or longer. 
The testing was conducted using an ASTM E274 towed friction trailer with a ribbed tire. Water was sprayed on the pavement in front of the trailer wheel while moving along the roadway. The wheel was then locked up, simulating a skid. The braking forces were measured and converted into a friction number, which correlates to the coefficient of wet sliding friction.

Typical ribbed tire friction values on various pavement types ranged from about 20 to 50 or slightly higher. Typical values for sand surfaces, formerly used to provide high friction values on interstates and other high speed roadways, were in the 50's. A friction value of 30 was considered a warning level; pavements with friction values below 30 were inspected to determine if remedial action were needed.

The friction values measured on this project are summarized in Tables 17 through 20 . Tables 17 through 19 show the friction values measured in each lane separately and Table 20 shows the overall average for each material in all three lanes. This data is presented graphically in Figures 26 through 29. The values have been normalized to account for variations in the vehicle speed from $40 \mathrm{mph}$ and for the ambient temperature. Since tests were taken approximately every 0.3 to $0.5 \mathrm{~km}(0.2$ to $0.3 \mathrm{mi}$.), the values in the table reflect the average of at least four tests, with more tests available on the last control section $(\mathrm{J})$ due to its length.

Table 17 Friction Numbers at $40 \mathrm{mph}$ in Driving Lanes

\begin{tabular}{|l|c|c|c|c|c|c|}
\hline Section & 1990 & 1991 & 1992 & 1994 & 1995 & 1996 \\
\hline A. Control & 38.6 & 41.4 & 40.2 & 35.3 & 33.6 & 35.0 \\
\hline B. PAC & 38.3 & 41.0 & 43.4 & 36.7 & 36.6 & 42.7 \\
\hline C. Fibers & 38.8 & 44.2 & 40.9 & 37.6 & 35.6 & 46.2 \\
\hline D. PE & 37.3 & 42.4 & 39.8 & 37.6 & 31.5 & 41.9 \\
\hline E. MGAC & 38.3 & 44.6 & 44.9 & 35.0 & 34.5 & 42.8 \\
\hline F. SBR & 36.6 & 45.4 & 48.2 & 42.2 & 35.1 & 45.4 \\
\hline G. Neo & 41.6 & 42.1 & 44.9 & 35.9 & 36.8 & 42.0 \\
\hline H. AR & 38.1 & 43.7 & 43.8 & 39.2 & 37.9 & 45.5 \\
\hline J. Control & 39.3 & 41.1 & 40.4 & 35.3 & 34.6 & 36.7 \\
\hline
\end{tabular}


Table 18 Friction Numbers at $40 \mathrm{mph}$ in Center Lanes

\begin{tabular}{|l|c|c|c|c|c|c|}
\hline Section & 1990 & 1991 & 1992 & 1994 & 1995 & 1996 \\
\hline A. Control & 37.4 & 43.8 & 43.2 & 35.5 & 33.8 & 38.0 \\
\hline B. PAC & 39.3 & 44.9 & 42.9 & 37.8 & 35.3 & 41.6 \\
\hline C. Fibers & 37.8 & 46.4 & 42.2 & 40.5 & 35.2 & 44.0 \\
\hline D. PE & 39.4 & 45.8 & 42.6 & 38.9 & 33.8 & 43.4 \\
\hline E. MGAC & 39.4 & 45.7 & 42.7 & 38.3 & 35.8 & 40.1 \\
\hline F. SBR & 38.1 & 45.3 & 42.9 & 40.1 & 35.7 & 44.1 \\
\hline G. Neo & 37.0 & 42.8 & 43.5 & 36.4 & 35.2 & 43.4 \\
\hline H. AR & 37.0 & 43.8 & 40.5 & 38.8 & 35.9 & 45.8 \\
\hline J. Control & 39.2 & 44.0 & 44.2 & 37.2 & 33.6 & 39.4 \\
\hline
\end{tabular}

Table 19 Friction Numbers at $40 \mathrm{mph}$ in High Speed Lanes

\begin{tabular}{|l|c|c|c|c|c|c|}
\hline Section & 1990 & 1991 & 1992 & 1994 & 1995 & 1996 \\
\hline A. Control & 39.9 & 44.8 & 47.2 & 43.2 & 39.9 & 49.1 \\
\hline B. PAC & 40.9 & 49.9 & 48.7 & 45.3 & 43.2 & 52.2 \\
\hline C. Fibers & 40.3 & 49.4 & 49.0 & 46.6 & 42.5 & 55.3 \\
\hline D. PE & 41.4 & 49.1 & 49.5 & 46.2 & 40.9 & 52.9 \\
\hline E. MGAC & 42.0 & 47.6 & 51.0 & 45.7 & 42.2 & 54.2 \\
\hline F. SBR & 40.4 & 48.6 & 47.4 & 47.9 & 43.8 & 55.3 \\
\hline G. Neo & 45.0 & 46.3 & 46.2 & 45.3 & 44.3 & 51.4 \\
\hline H. AR & 40.6 & 45.2 & 47.0 & 45.0 & 43.9 & 53.5 \\
\hline J. Control & 42.7 & 48.9 & 46.5 & 45.4 & 41.6 & 49.4 \\
\hline
\end{tabular}


Table 20 Overall Average Friction Numbers at $40 \mathrm{mph}$

\begin{tabular}{|l|c|c|c|c|c|c|}
\hline Section & 1990 & 1991 & 1992 & 1994 & 1995 & 1996 \\
\hline A. Control & 38.6 & 43.3 & 44.0 & 38.1 & 36.8 & 40.7 \\
\hline B. PAC & 39.3 & 44.9 & 45.0 & 40.0 & 38.1 & 45.5 \\
\hline C. Fibers & 39.0 & 46.7 & 44.0 & 41.6 & 38.1 & 48.5 \\
\hline D. PE & 39.4 & 45.8 & 44.0 & 40.5 & 35.5 & 46.0 \\
\hline E. MGAC & 39.9 & 46.6 & 46.2 & 40.0 & 37.3 & 45.4 \\
\hline F. SBR & 38.4 & 46.4 & 45.7 & 43.6 & 38.1 & 48.5 \\
\hline G. Neo & 41.2 & 42.8 & 44.8 & 39.1 & 38.6 & 46.1 \\
\hline H. AR & 38.6 & 44.2 & 42.7 & 41.6 & 39.0 & 48.3 \\
\hline J. Control & 40.3 & 44.7 & 43.7 & 39.7 & 36.5 & 41.7 \\
\hline
\end{tabular}

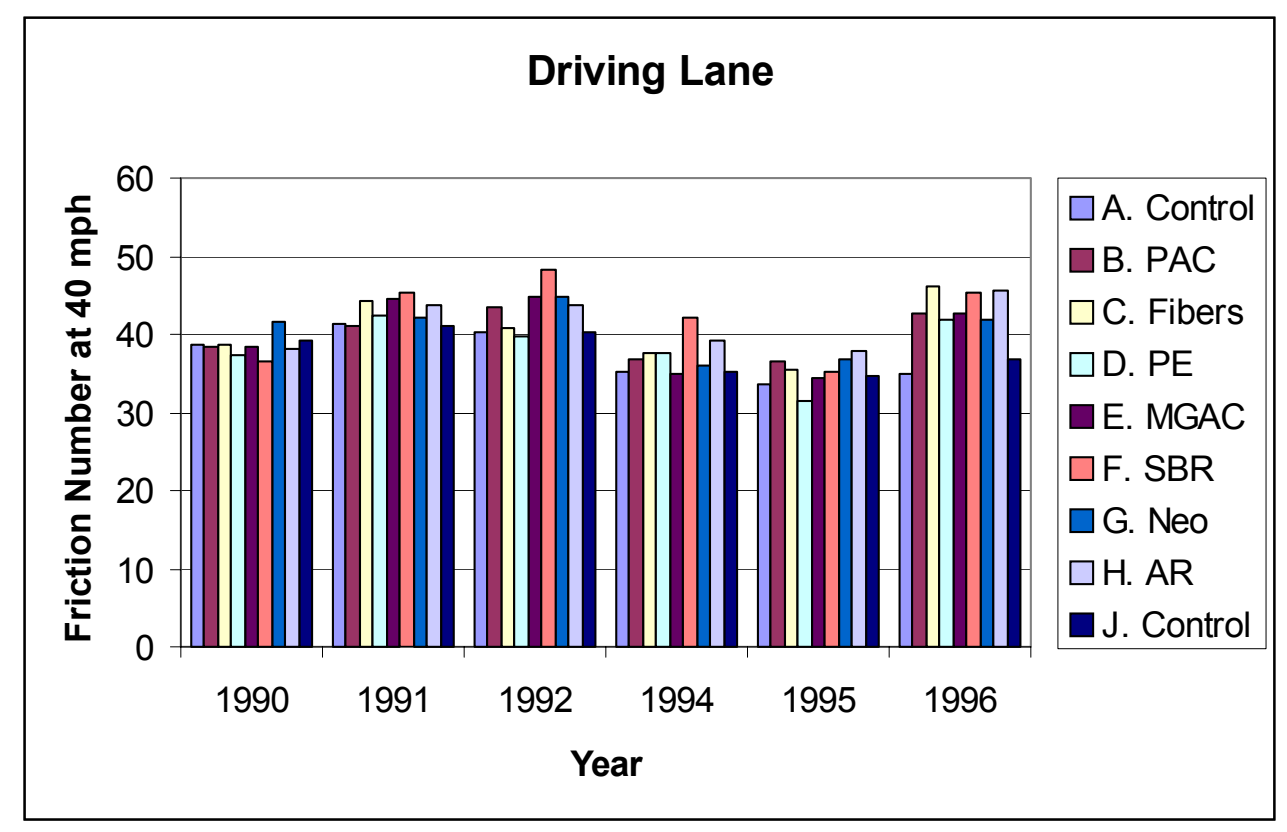

Figure 26 Average Friction Number vs. Time in Driving Lane 


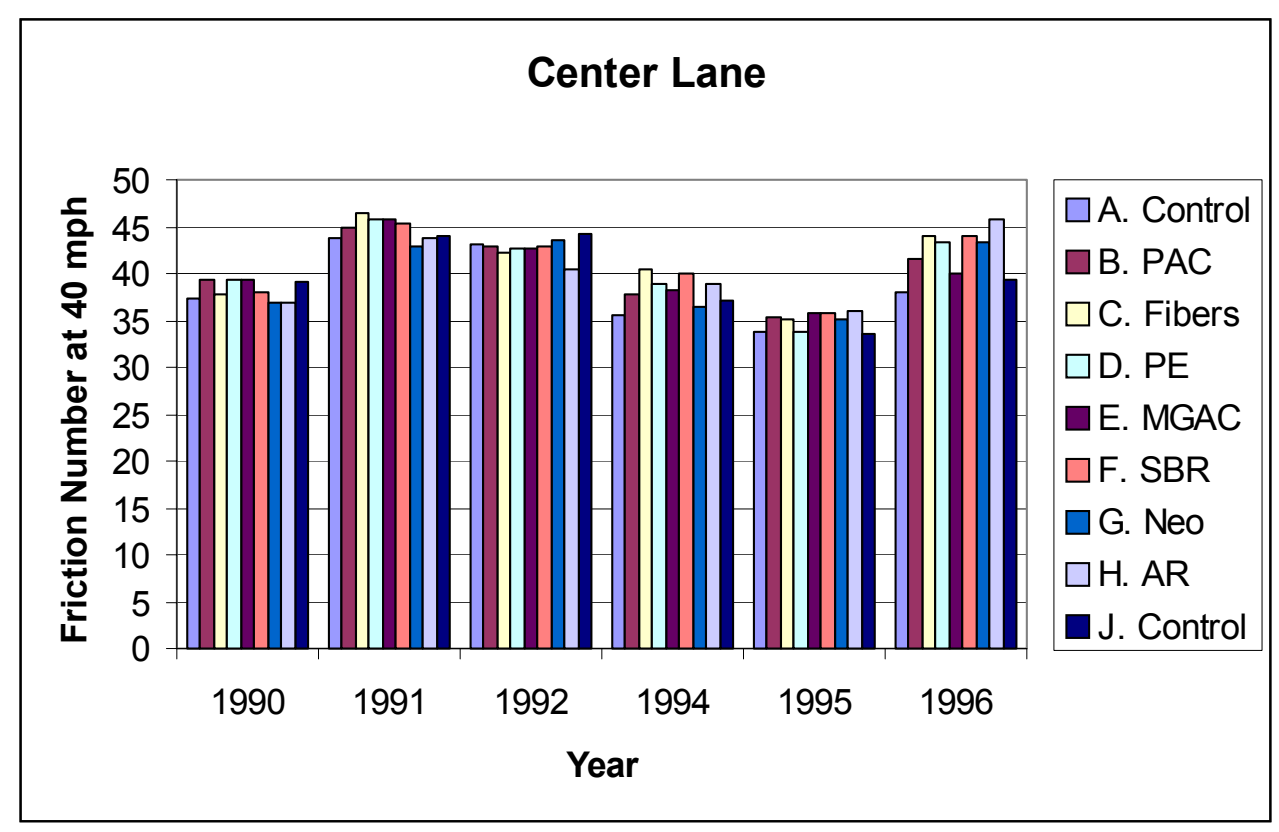

Figure 27 Average Friction Number vs. Time in Center Lane

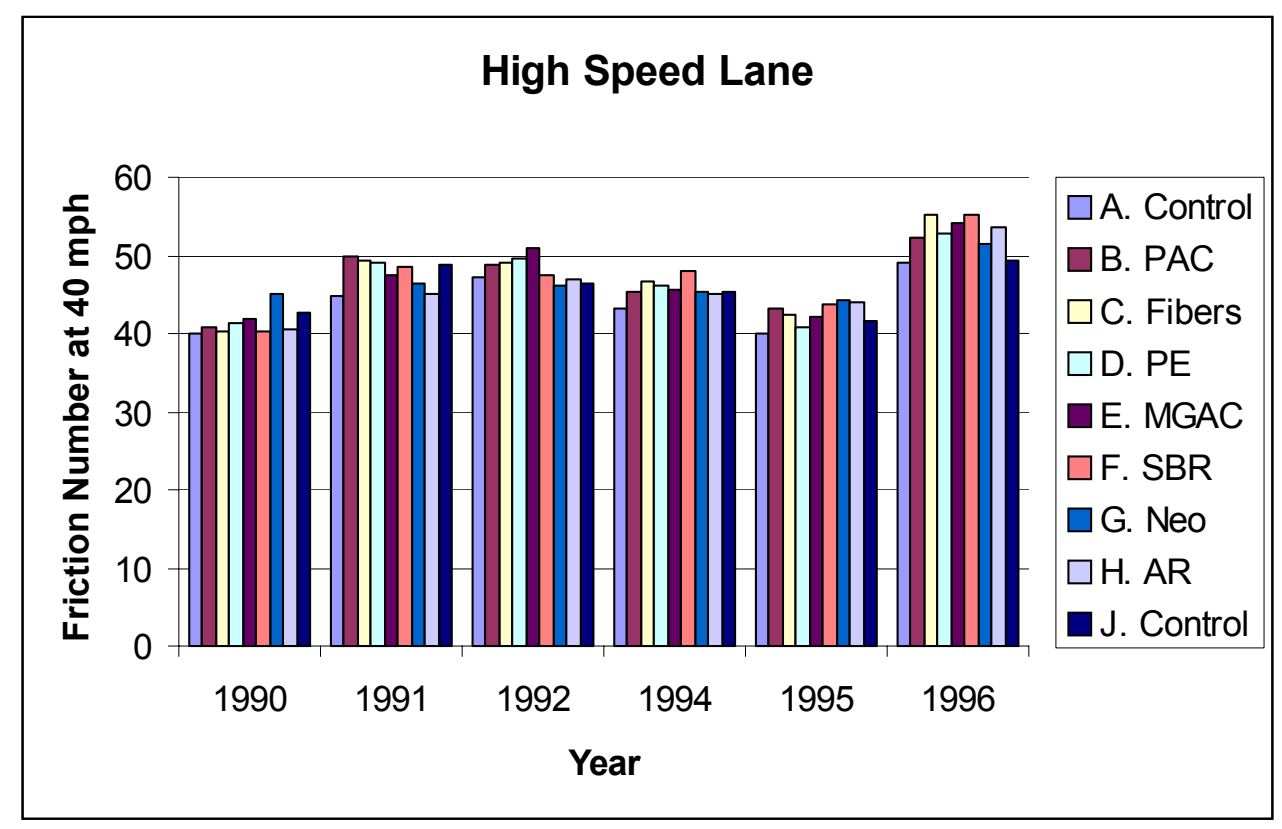

Figure 28 Average Friction Number vs. Time in High Speed Lane 


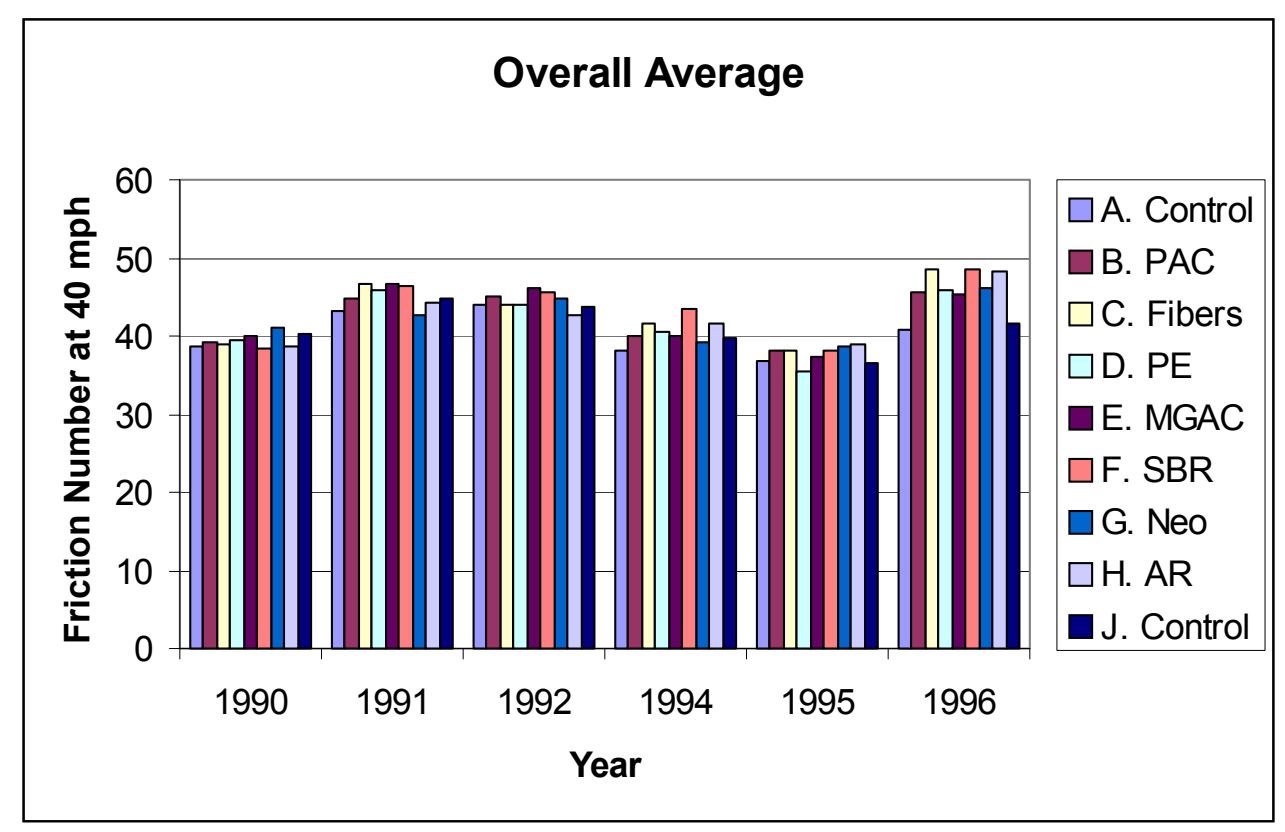

Figure 29 Overall Average Friction Number vs. Time (All Lanes)

Friction data was collected in 1993, but the tests were only conducted over a length of 2.5 to 2.8 miles. This is likely due to traffic control set up at the southern end of the project for construction in an adjacent area. It is not possible, however, to conclusively determine the starting point for the testing, so this data was omitted from analysis.

All friction testing was performed in the Fall in an attempt to reduce seasonal variation as much as possible. Some variation is unavoidable, however. For example, all of the values measured in 1996 are higher than the values in the previous two years. Perhaps this testing was conducted after a significant rainfall event cleaned the surface. The relative comparisons of the test sections in 1996 are still valid, but the overall increase in friction number is likely an aberration.

The 1997 Friction Inventory and 2001 special testing both indicate that the test sections are still performing quite well in terms of friction. The friction numbers for this part of I-465 were between 30 and the high 40's with the smooth tire, which would correspond roughly to 4050 with a ribbed tire. (The warning level for the smooth tire is 20 , well below the observed values.)

In general terms, differences of three or four friction numbers are not considered significant, due to the typical testing variability. For the most part, then, the friction numbers do 
not show significant differences between the test sections. There are, however, some interesting observations that can be made about this data.

As expected, the friction numbers measured in 1990, approximately two months after placement of the surface courses, are lower than the values measured in 1991 and 1992. After traffic wore off the binder film coating, the friction values increased. The relative friction levels between test sections in 1990 were essentially the same. Apparently, then, the different binders do not have significantly different frictional properties. No significant differences were noted in 1991 either.

In 1992, slight differences began to appear between the sections. The control sections had lower friction values than most of the other materials on a consistent basis over the following years. The values for the PE section were similar to the control sections except in 1996, when the PE section had a significantly higher friction value than the control. The MGAC section also had a friction value as low as the control sections, but only in 1994 in the driving lane. In the other lanes, as well as in 1992, 1995 and 1996, the MGAC section exhibited a higher friction value. This implies that the 1994 driving lane data was questionable; perhaps the area tested was contaminated.

The effect of traffic on friction level is quite apparent. All three lanes had approximately the same friction level in 1990. From 1991 on, however, the high speed lane, which had less traffic than the other lanes, had higher friction levels. The friction values in the high speed lane were 5 to 10 points higher than the center or driving lanes.

Overall, the modified sections appeared to have slightly higher friction values than the control sections, except as noted above. The 1996 data showed all of the modified sections having significantly higher friction values than the control sections. (Although the magnitude of the values in 1996 seems unreasonable, a comparison between sections is still likely valid.) Perhaps the control sections have lost aggregate and macrotexture, while the modified binders have held on to the aggregate better, thereby retaining macrotexture. There are ways to measure macrotexture, including the sand patch test and a comparison of ribbed and smooth friction tire test results. Unfortunately, however, we do not have baseline data from earlier in the life of the pavement for comparison.

An analysis of variance was performed on the friction data from 1990 through 1996 looking at the friction number as a function of material, year and lane using SAS, a commonly used statistics program. This SAS analysis showed that all of the factors were significant. 
Friction certainly would be expected to vary by year and by lane due to the variation in traffic.

The fact that the material type was also significant lead to a more detailed analysis.

SAS was used to analyze the 1996 data lane by lane. A comparison of means (Duncan) was performed to determine which sections had similar friction levels and which were performing differently. No differences were noted between the northbound or southbound control sections in any lane, which suggests that the traffic does not vary significantly by direction. In the driving and middle lanes, which carry most of the traffic, the modified sections could not be distinguished from one another, but they were significantly different from the control sections. That is, the control sections were in one group and the modified sections were in another group. The modified sections did provide a higher friction level than the control sections, as suggested by the graphical data in Figures 26 through 29.

In the high speed lane, which experiences less traffic, there were three overlapping groups. Group A, with the highest friction values, included the Fiber, SBR, MGAC, AR, PE and PAC mixes, in order of the mean value. The second group included the MGAC, AR, PE, PAC and Neoprene. The third group, with the lowest means, included the PAC, Neoprene and Control sections. Sections within a group are not significantly different from each other. The reason for these groupings is not obvious. In general, though, it does appear again that most of the modifiers are providing slightly higher friction levels than the control sections. The control sections are only grouped with the PAC and Neoprene.

None of the sections, not even the control sections, are experiencing friction problems. The difference in friction levels between the modified and control sections, however, is an interesting and somewhat unexpected side benefit of the use of modifiers. 


\subsection{Roadway Management Data}

Since traffic control considerations would not permit detailed visual inspections and manual rut depth measurements in the middle and driving lanes, INDOT's Roadway Management database was searched for information about this section of I-465. This data supplements and supports the detailed information about the high speed lane.

The management system showed that the overall roughness, as measured by International Roughness Number (IRI) was 74 northbound and 76 southbound in 1999. For comparison purposes, smooth new concrete pavements in Indiana typically have IRI values around 100. The smoothest new hot mix asphalt pavements have ISI values in the high 50's to 60's. These values for an 11 year old overly aver concrete, then, are considered quite good.

The average rut depth in both directions was $3 \mathrm{~mm}(0.13 \mathrm{in}$.), which is extremely good for an overlaid pavement of this age under heavy traffic. At the time this pavement was constructed, premature rutting was a significant problem in Indiana. The Pavement Condition Rating (PCR) in 1999 was 89 northbound and 87 southbound, both of which are considered on the high side of the good category.

The Roadway Management data is collected on digital video and stored on tape. The 1998 tapes were reviewed to subdivide the contract into the individual test sections. Rut depth and IRI are collected continuously throughout the project, but PCR is only determined on the first $500 \mathrm{ft}$. of every mile of roadway. For this reason, it is impossible to determine the PCR for each half-mile control section. Nonetheless, the data was reviewed to determine if there were obvious differences between the sections. The IRI varied from 66 to 92 , rut depth from 0.04 to 0.08 in. and PCR from 85.5 to 90.5 . None of these are considered sizeable differences. This indicates that although there is a noticeable difference in the cracking patterns between the sections, as discussed in 5.2, this has not yet impacted the overall serviceability of the roadway. The data also shows that rutting is negligible in all sections and all lanes. 


\section{LABORATORY TEST RESULTS}

Initially, this study was designed to look at both rutting and cracking distresses. Over ten years of field performance, however, have shown that no significant rutting has occurred. The aggregates used in the mixtures are all the same and the gradations are largely the same, with a few deviations for particular binders. The gradations conform to the requirements for Superpave mixtures, for the most part. Apparently the high quality aggregates, combined in appropriate gradations, coupled with close attention to detail during construction, resulted in high quality mixtures that are resistant to rutting. Since rutting is generally acknowledged to be a distress that occurs early in the life of a pavement, before the binder has age-hardened, it is unlikely that rutting will develop now.

The primary distress type noted on any of the field inspections is cracking. Significant differences in the rate of crack development and severity of the cracking do exist. The cracking largely appears to be reflective cracking, perhaps exacerbated by thermal contractions. Reflective cracking alone does not appear to explain the observed distresses, since the cracking pattern is not consistent with observed crack and joint spacing of the underlying pavement. The state of repair of the existing concrete pavement was uniform throughout the project length; undersealing and concrete patching were performed to stabilize the concrete pavement before overlaying. Brittleness of some of the binders appears to be a factor.

The focus of the testing, then, turned towards tests related to cracking behavior since this was the predominant distress type noted in the field. Limited testing of rutting parameters was conducted earlier in the project, but this was largely dropped in the later years since no rutting had developed.

\subsection{Binder Testing}

During construction, samples of the binders were obtained and tested to determine if they met the specification requirements in effect at the time. All of the binders met the specifications, except as noted in Chapter 4.

Following construction, samples of the modified binders were retained in sealed cans in the laboratory. Development of the Superpave binder specifications began soon after this project 
was constructed, and in the mid-1990's the specifications were changing rapidly. Because the available samples were quite limited, testing the binder properties was postponed until the binder specifications had stabilized.

Limited high temperature binder testing was performed on the binders under the original Superpave binder testing protocols. These results are summarized in Table 21. This testing is now not considered appropriate for most modified binders because modification often causes the binders to violate one of the underlying assumptions of the Superpave specification, typically linearity.

In 1996, Dr. Hussain Bahia and the Asphalt Institute began research into testing modified binders in the Superpave system, as described in Chapter 2. Further testing of the samples collected under this project was again put on hold awaiting the recommendations of Bahia's work.

In late 1999, Dr. Bahia and his students at the University of Wisconsin performed binder testing on samples of most of the modifiers used in this research as partial field validation of their NCHRP 9-10 findings. Because the predominant distress observed on I-465 is cracking, not rutting, Dr. Bahia's testing focused on the low temperature properties of the binders.

\subsubsection{Dynamic Shear Rheometer Testing}

Dynamic shear rheometer testing was conducted at the North Central Superpave Center on retained samples of the binders according to standard protocols (AASHTO TP5, Method for Determining the Rheological Properties of Asphalt Binder Using a Dynamic Shear Rheometer). The binders had been stored in the laboratory in sealed quart cans for over ten years. These binders were tested as original binders. The results are presented in Table 21 . 
Table 21 Average $G^{*} / \sin \delta$ for Original Binders

\begin{tabular}{|c|c|c|c|c|c|c|}
\cline { 2 - 7 } \multicolumn{1}{c|}{} & \multicolumn{6}{c|}{ Average G*/sin $\delta$} \\
\hline Temperature, ${ }^{\circ} \mathrm{C}$ & AC-20 & PAC & MGAC & PE & Neo & AR \\
\hline 52 & 6.72 & 7.82 & & & 5.61 & \\
\hline 58 & 2.78 & 3.76 & 5.83 & & 2.06 & \\
\hline 64 & 1.17 & 1.84 & 2.11 & 9.21 & 0.88 & 6.60 \\
\hline 70 & 0.60 & 1.00 & 1.21 & 4.60 & 0.47 & 5.00 \\
\hline 76 & & 0.67 & 0.62 & 2.64 & & 4.13 \\
\hline 82 & & & & 1.72 & & 3.84 \\
\hline 88 & & & & 0.83 & & 3.74 \\
\hline Critical High & & & & & & \\
Temp., ${ }^{\circ} \mathrm{C}$ & 65.8 & 70.0 & 72.1 & 86.8 & 63.4 & $<88$ \\
\hline High PG Grade & PG64 & PG70 & PG70 & PG82 & PG58 & PG88 \\
\hline
\end{tabular}

The SBR could not be tested because of the way the modifier was added to the base asphalt. Since the modifier was pumped directly into the AC line downstream of any sampling ports, no samples of the modified binder were available.

The critical temperature shown in Table 21 is the temperature at which the binder just meets the high temperature specification limit of $1.00 \mathrm{kPa}$ for $\mathrm{G}^{*} / \mathrm{sin} \delta$ on original binder. This data shows that the high critical temperatures of the different binders vary widely. The Neoprene had the lowest critical temperature of $63.4^{\circ} \mathrm{C}$. The Asphalt Rubber had the highest critical temperature, which was in excess of $88^{\circ} \mathrm{C}$. This data may not be reliable for two reasons. First, this type of testing may not be appropriate for modified binders, as pointed out in Chapter 2.

Second, the effects of storage on these binders cannot be determined. Some may have aged more than others. None of the mixtures produced with these binders have exhibited rutting in the field, so the apparent variation in critical high temperature is of questionable importance.

Intermediate temperature testing was not completed, so the grades shown in Table 21 are based only on the high temperature data. This testing was not done due to the relatively small amounts of binder remaining and the current state of controversy over how fatigue testing is done. It appears likely that the fatigue parameter may change in the next few years. 


\subsubsection{Low Temperature Binder Testing Protocols and Results}

Dr. Bahia and his students performed the new low temperature test procedures and analysis proposed in NCHRP 9-10 on retained samples of the binders used in this field project. This was done in part to provide some validation of the methods they had developed.

Samples of four of the experimental binders and the control binder were tested at the University of Wisconsin. The SBR could not be tested since samples of the blended binder were not available. The Asphalt Rubber and Fibers were not tested because of their particulate nature.

This testing included Direct Tension at multiple temperatures and rates of strain, bending beam rheometer testing at multiple temperatures and glass transition behavior using a specially designed dilatometer. The direct tension results are shown in Table 22 and an example of the glass transition testing for the control binder is shown in Figure 30. The results in Table 22 include failure stress, as required by AASHTO PP42, Practice for Determination of LowTemperature Performance Grade(PG) of Asphalt Binders, and MP1a, Specification for Performance Graded Binder, and also failure strain, as recommended by the NCHRP 9-10 protocols. The combined data of the direct tension, bending beam, and the glass transition were used to estimate critical cracking temperature based on failure stress following the AASHTO PP42 and MP1a protocols. They were also used to estimate critical cracking temperatures based on failure strain using the NCHRP 9-10 protocols.

Following the protocols outlined in NCHRP 9-10, the University of Wisconsin team developed the failure master curves for each binder at a reference strain rate of $3 \% / \mathrm{min}$. These curves were developed based on the results of direct tension testing over a range of temperatures. The data is shown in Table 22 and Figure 31.

The thermal stresses that may build up in the pavement are estimated based on bending beam rheometer testing. A pavement constant of 18 is used to convert the thermal stresses in the BBR to the thermal stresses in the pavement. Table 23 and Figure 32 show the critical temperatures for the tested binders.

The failure master curves show that all of the tested modifications increase the failure strain at a given temperature with the exception of the Novophalt binder. The failure stresses of the PAC and Neoprene are similar. The MGAC has lower failure stresses. The AC-20 control exhibits failure stresses that are lower than the MGAC but higher than the Novophalt. The PAC and Neoprene exhibit the highest failure strains at any given temperature. The MGAC failure 
strains are intermediate, then the Novophalt and AC-20 binders exhibit the lowest failure strains at any temperature.

The new low temperature binder testing protocols were able to identify the test sections that exhibited the most cracking in the field. Failure strains and stresses would be expected to correlate with cracking. To investigate the specific relationships between the low temperature testing results of the binders and actual cracking observed, Figure 33 shows the correlation of cracking observed in 2001 and the stress and strain at failure at $-12 \mathrm{C}$ and $1 \mathrm{~mm} / \mathrm{min}$. This was the only temperature and strain rate for which a complete set of data for all binders were measured. As shown in part (a) of the figure the cracking count in the field appears to correlate highly with failure strain but less with failure stress. Using the critical temperatures estimated, the plot in part (b) of the figure was prepared. The correlations are much better in both strain and stress cases. The correlation to critical temperatures based on stress is $81 \%$ and to critical temperatures based on strain is $95 \%$.. 
Table 22 Low Temperature Binder Testing Under Revised Protocols

\begin{tabular}{|c|c|c|c|c|c|c|c|c|c|c|c|c|c|c|c|c|c|}
\hline \multirow{2}{*}{\multicolumn{2}{|c|}{$\begin{array}{l}\text { Temperature, }{ }^{\circ} \mathrm{C} \\
\text { Strain Rate, } \% / \min \end{array}$}} & \multicolumn{3}{|c|}{$0 \mathrm{C}$} & \multicolumn{4}{|c|}{-6} & \multicolumn{4}{|c|}{-12} & \multicolumn{3}{|c|}{-18} & \multicolumn{2}{|c|}{-24} \\
\hline & & 1 & 3 & 10 & 0.3 & 1 & 3 & 10 & 0.3 & 1 & 3 & 10 & 0.3 & 1 & 3 & 0.3 & 1 \\
\hline \multirow[t]{2}{*}{ AC-20 } & Stress, $\mathrm{MPa}$ & & & 1.35 & 0.89 & 1.35 & 1.89 & 2.34 & 1.53 & 1.91 & 2.42 & & & & & & \\
\hline & Strain, $\%$ & & & 4.92 & 5.34 & 4.92 & 3.35 & 2.30 & 0.72 & 0.46 & 0.39 & & & & & & \\
\hline \multirow[t]{2}{*}{ MGAC } & Stress, MPa & & & & & 1.12 & 1.80 & 2.71 & 1.32 & 2.22 & 2.56 & & 1.74 & & & & \\
\hline & Strain, $\%$ & & & & & 7.95 & 6.08 & 4.36 & 2.06 & 1.49 & 1.01 & & 0.65 & & & & \\
\hline \multirow[t]{2}{*}{$\mathrm{Neo}$} & Stress, MPa & & & & & & & 2.00 & 1.11 & 1.65 & 2.07 & 3.48 & 2.37 & 2.75 & 3.41 & & \\
\hline & Strain, \% & & & & & & & 14.60 & 7.27 & 5.52 & 2.23 & 1.11 & 1.54 & 0.80 & 0.24 & & \\
\hline \multirow[t]{2}{*}{$\mathrm{PE}$} & Stress, MPa & 0.98 & 1.32 & 2.07 & 0.52 & 1.51 & & & 1.61 & 2.11 & & & & & & & \\
\hline & Strain, \% & 12.16 & 9.82 & 7.01 & 5.31 & 3.46 & & & 1.54 & 0.96 & & & & & & & \\
\hline \multirow[t]{2}{*}{$\overline{\mathrm{PAC}}$} & Stress, $\mathrm{MPa}$ & & & & & & & & & 1.68 & 2.39 & 3.42 & 2.12 & 3.09 & 3.60 & 2.72 & 3.66 \\
\hline & Strain, $\%$ & & & & & & & & & 7.42 & 6.87 & 4.20 & 3.01 & 2.58 & 1.33 & 0.95 & 0.74 \\
\hline
\end{tabular}




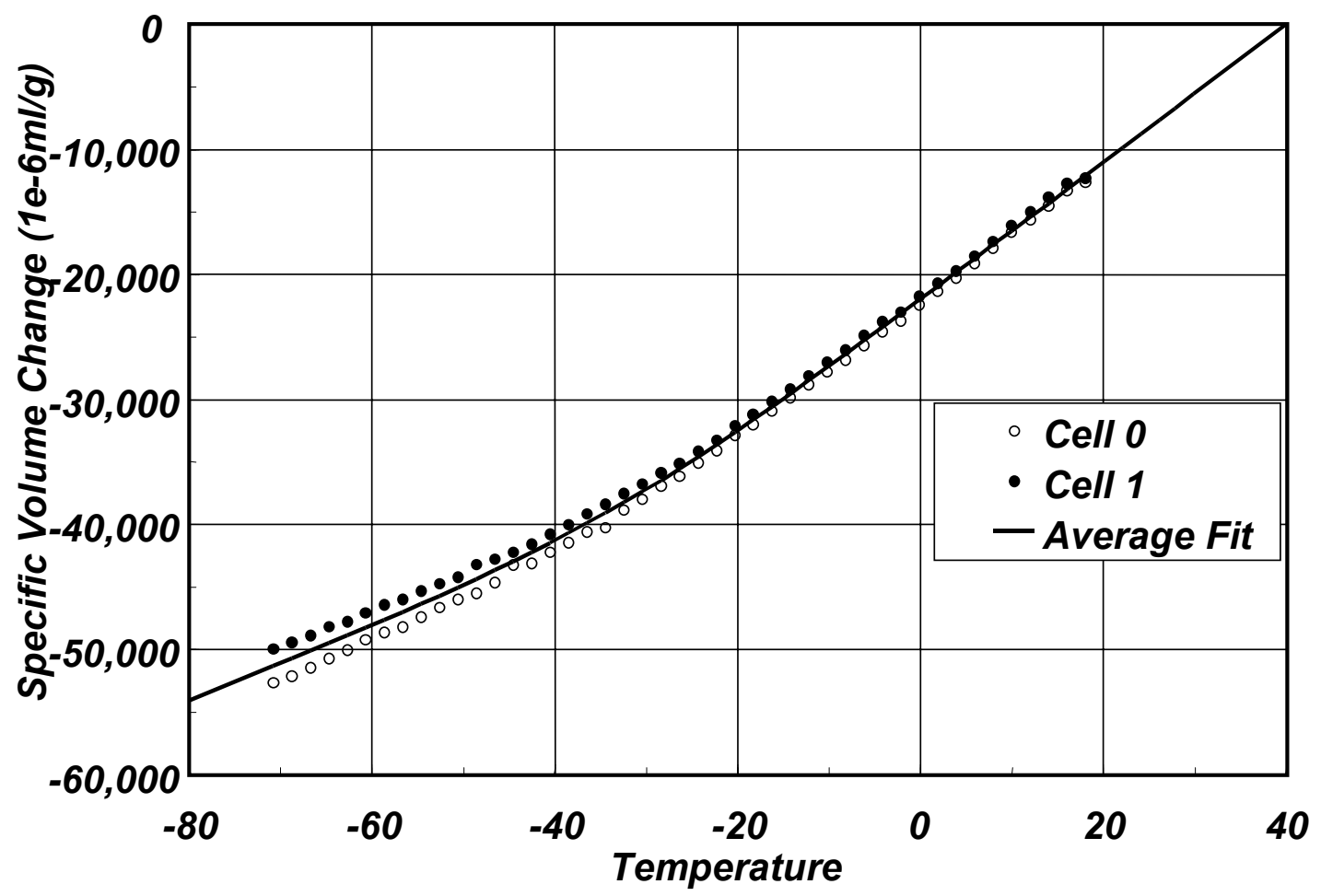

Figure 30 Typical Glass Transition Temperature Results Measured Following NCHRP 9-10 Protocols 
Failure Strain Properties of Purdue Binders

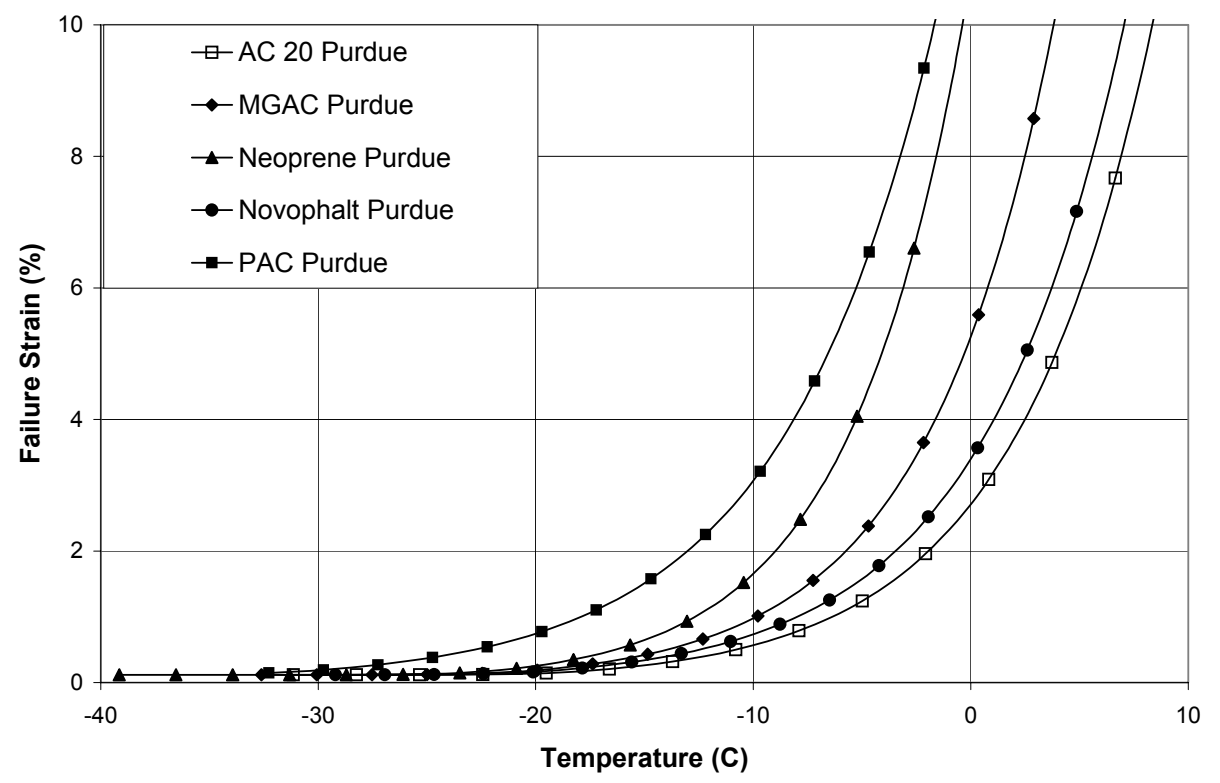

(a) Failure Strain Master Curves

Failure Stress Properties of Purdue Binders

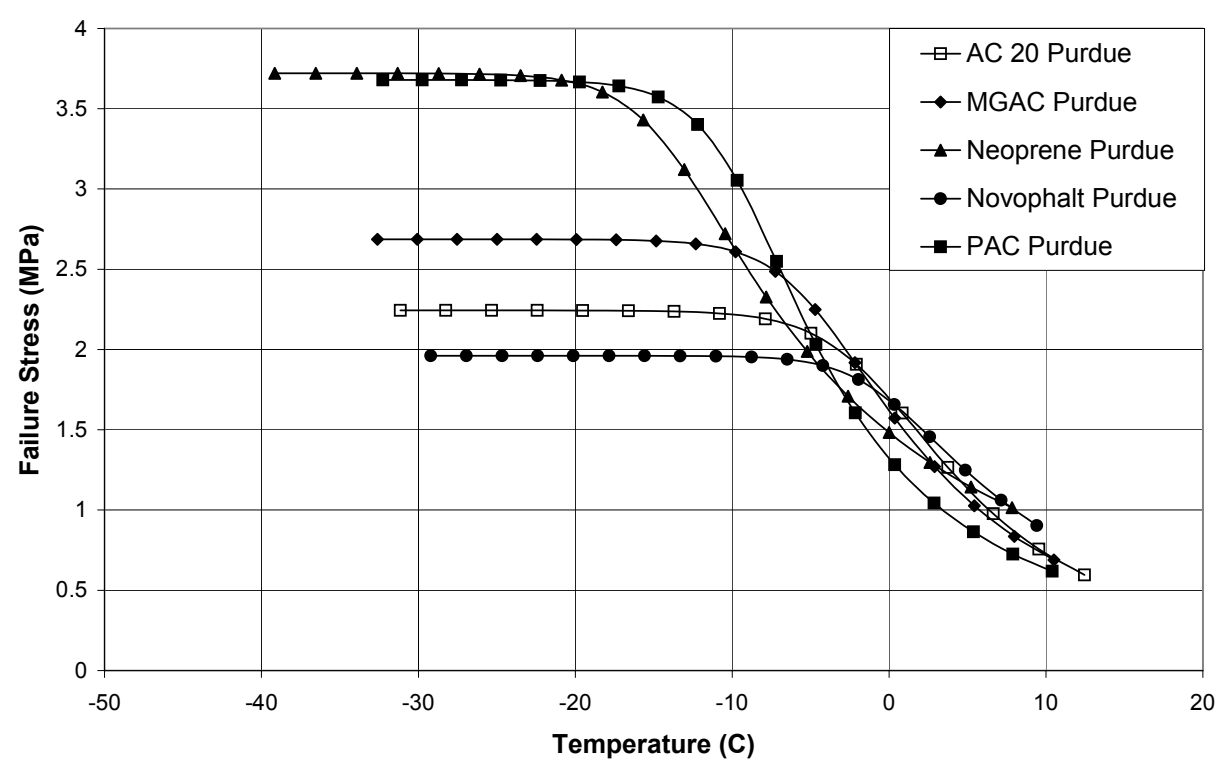

(b) Failure Stress Master Curves

Figure 31 Effect of Modification on Failure Strain Properties 


\section{Critical Cracking Temperatures of Purdue Binders}

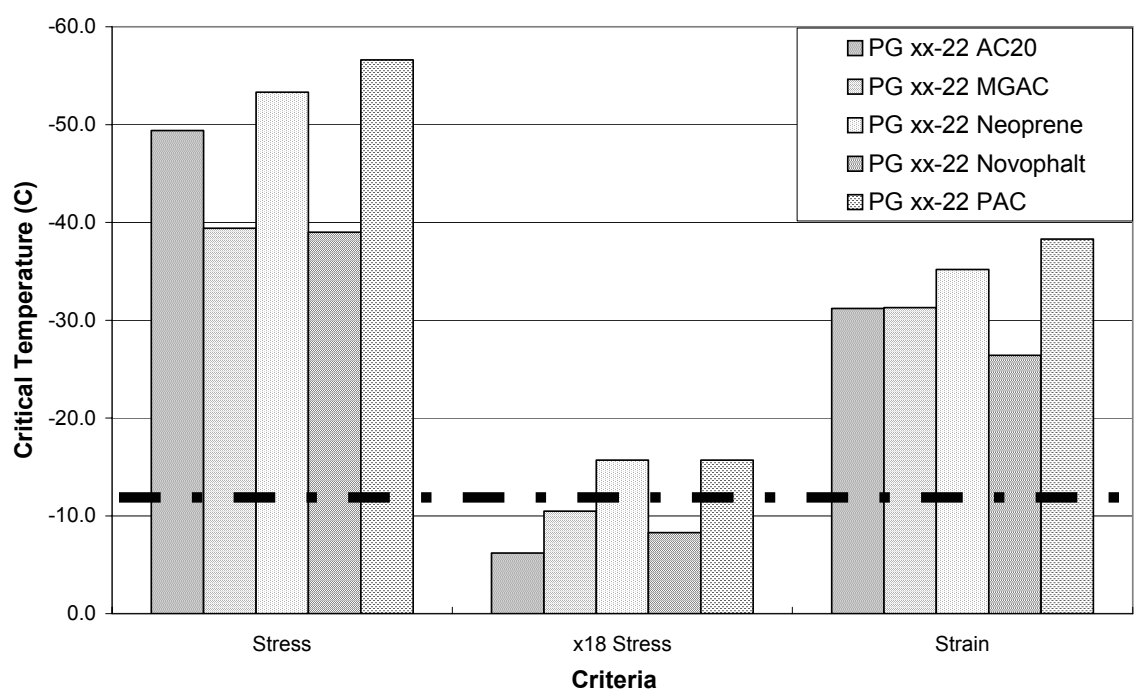

Figure 32 Critical Cracking Temperatures

Table 23 Estimates of Critical Cracking Temperature for Modified Binders

\begin{tabular}{|l|c|c|c|c|}
\hline \multirow{2}{*}{ Binder } & \multirow{2}{*}{$\begin{array}{c}\text { Cooling Rate } \\
\end{array}$} & \multicolumn{3}{|c|}{ Critical Temperatures $\left({ }^{\circ} \mathrm{C}\right)$} \\
\cline { 3 - 5 } & 1 & -49.4 & -6.2 & -31.2 \\
\hline AC-20 & 1 & -39.4 & -10.5 & -31.3 \\
\hline MGAC & 1 & -53.3 & -15.7 & -35.2 \\
\hline Neoprene & 1 & -39.0 & -8.3 & -26.4 \\
\hline Novophalt & 1 & -56.6 & -15.7 & -38.3 \\
\hline PAC & & & & \\
\hline
\end{tabular}




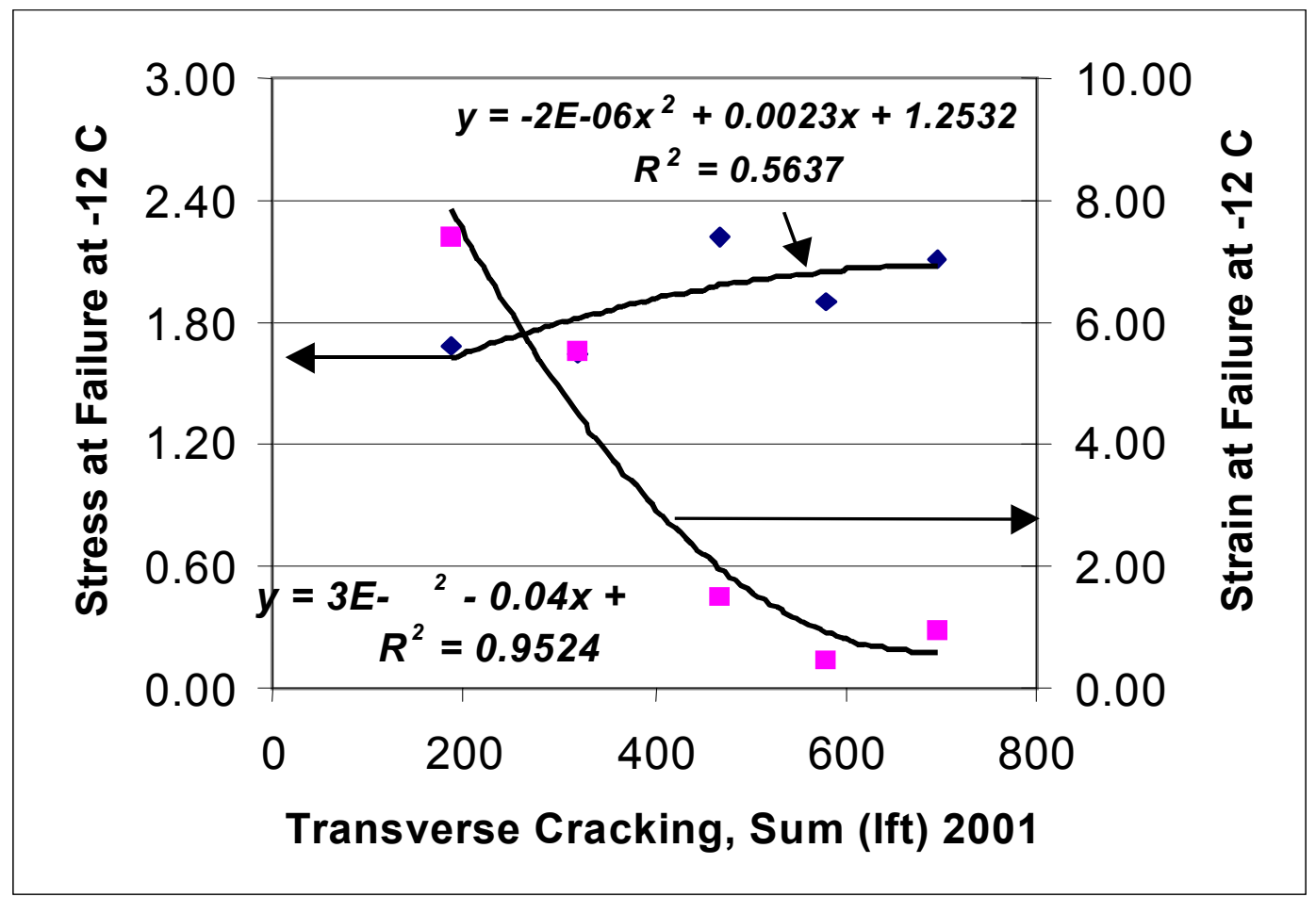

a. Correlation with stress and strain values

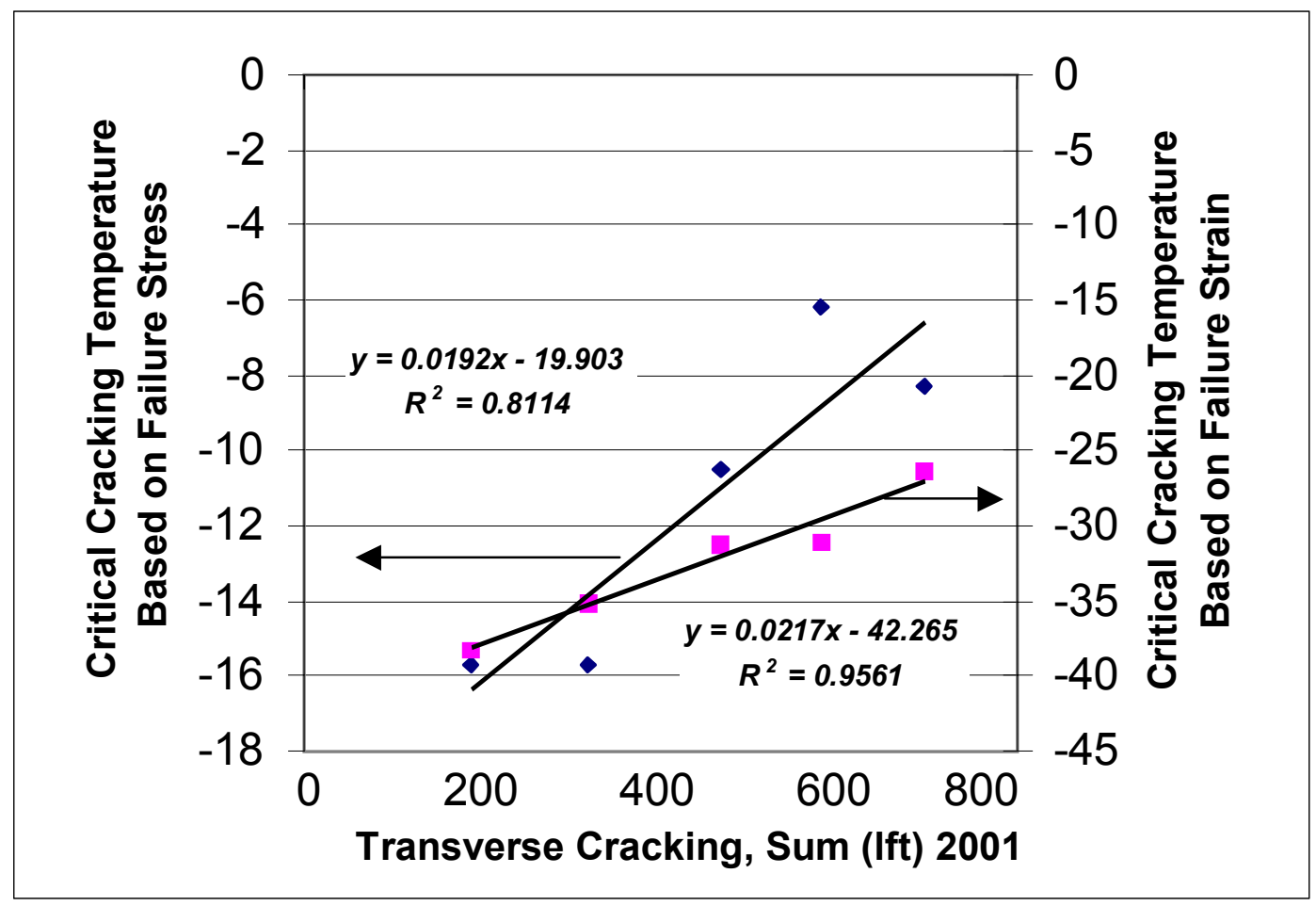

b. Correlations with Critical Temperatures

Figure 33 Correlations of Cracking Counts Measured in 2001 with Direct Tension Results and Critical Cracking Temperatures 
The testing conducted following the MP1a and PP42 protocols is quite involved and time consuming. It requires, at a minimum, testing in the BBR at two temperatures and the DTT at one temperature. Conventional MP1 testing, on the other hand, requires only one set of tests in the BBR. States are currently considering MP1a and whether the testing adds value. The data from this experiment was therefore examined to determine how the binders compared based on MP1 testing as well as MP1a.

The results of the BBR low temperature testing are shown in Table 24. In Figures 34 and 35 that data is plotted against the observed field cracking. This data shows that the control and PE binders both grade out as -16 grade material, the MGAC and Neoprene grade as -22 and the PAC grades as a -28 . The figures show that there is a good correlation between low critical temperature and observed cracking. That is, as the critical temperature increases (becomes less megative) the observed cracking increases.

Table 24 Binder Low Critical Temperatures by MP1

\begin{tabular}{|c|c|c|c|}
\hline \multirow{2}{*}{ Binder } & \multicolumn{2}{|c|}{ Critical Temperatures $\left({ }^{\circ} \mathrm{C}\right)$} & \multirow{2}{*}{ Low PG Grade } \\
\cline { 2 - 3 } & $\mathrm{S}(60)=300 \mathrm{MPa}$ & $\mathrm{m}(60)=0.300$ & -16 \\
\hline AC20 Control & -16.8 & -11.4 & -22 \\
\hline MGAC & -17.9 & -14.1 & -22 \\
\hline Neo & -17.6 & -15.6 & -16 \\
\hline PE & -13.9 & -7.3 & -28 \\
\hline PAC & -19.9 & -17.5 & \\
\hline
\end{tabular}

Thus, while the MP1a testing may be preferred from a theoretical point of view, in this particular case, it does not appear to offer a great improvement in the ability to predict cracking compared to the simpler MP1 testing. This is based on limited data at one site with only a few binders. Additional efforts are underway on a national level to verify the need to implement MPla testing. 


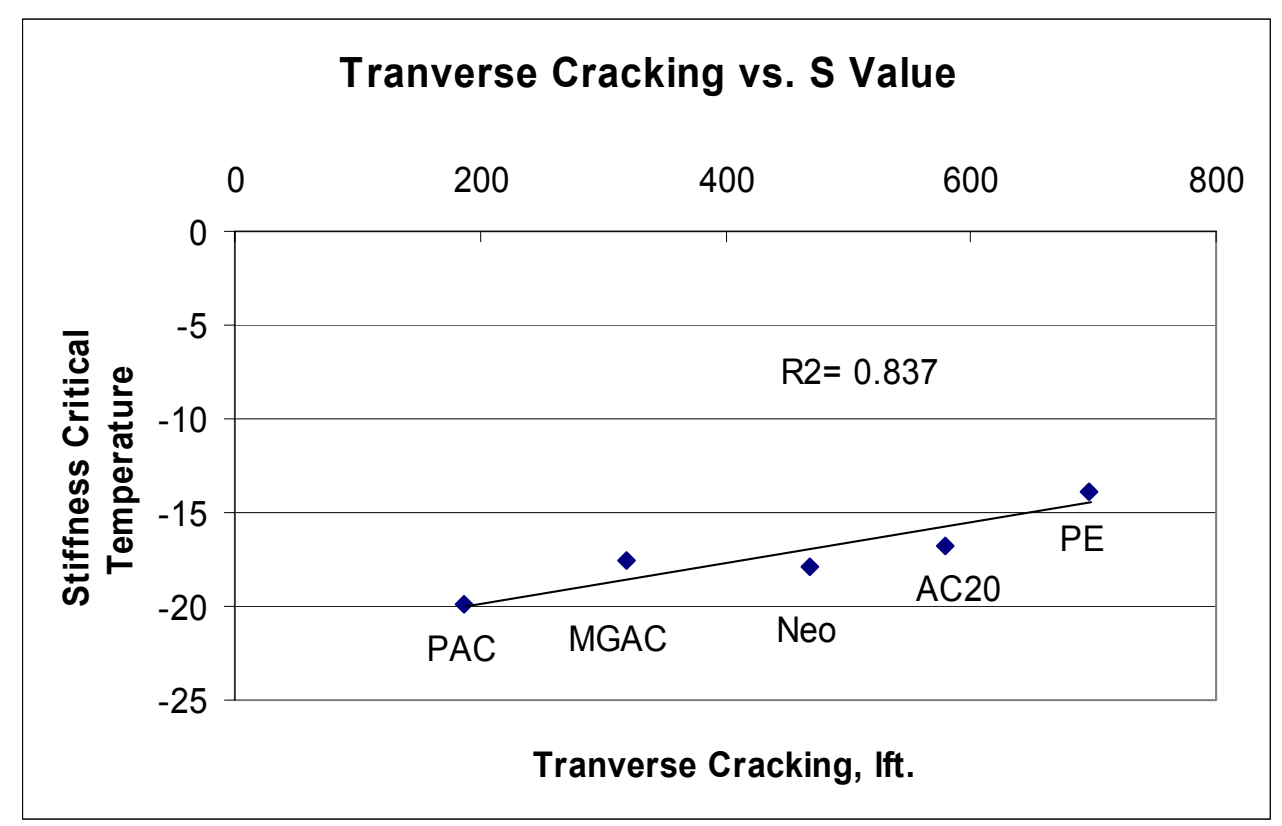

Figure 34 Critical Temperature by S Value vs. Cracking

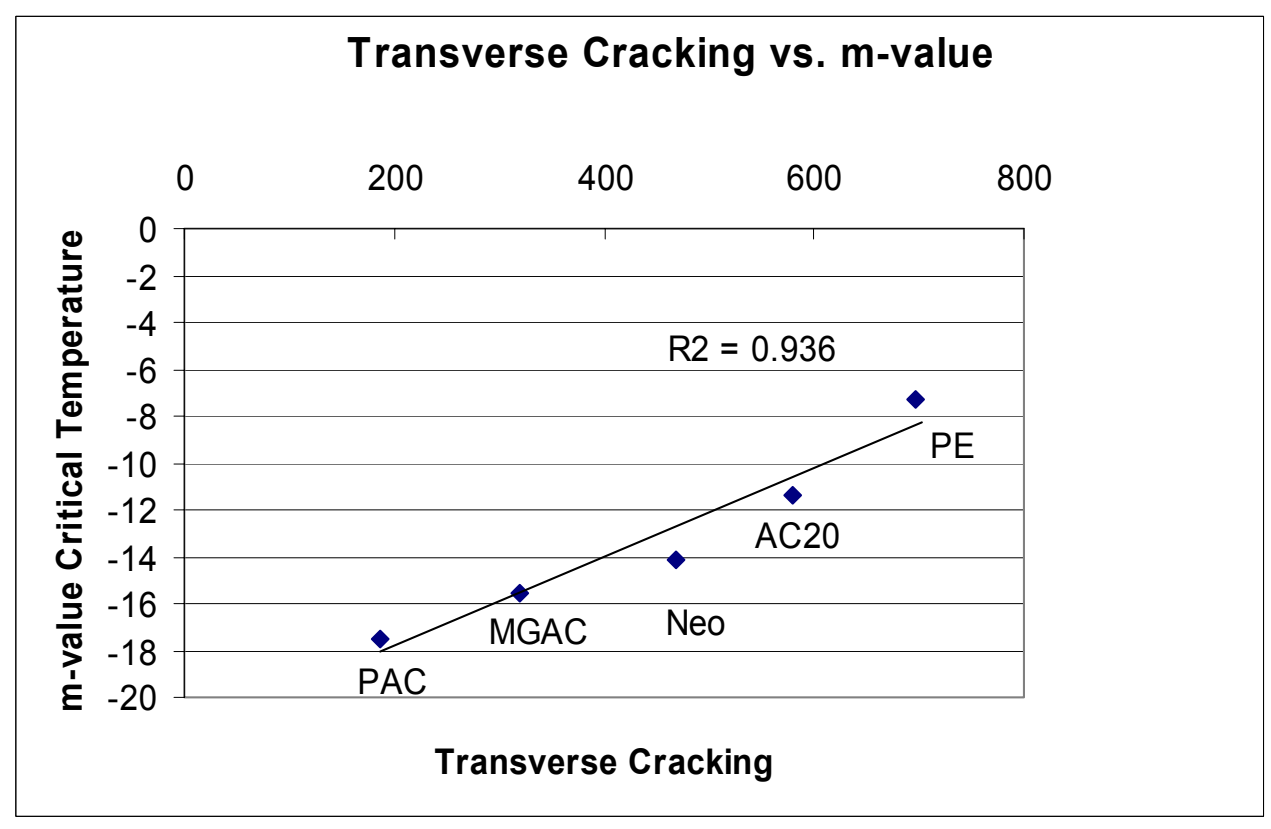

Figure 35 Critical Temperature by m-value vs. Cracking 


\subsection{Mixture Testing}

This section describes the tests performed on retained samples of the plant-produced mixtures. This testing includes loaded wheel testing and indirect tensile testing.

\subsubsection{Hamburg Wheel Testing of Field Cores}

Wheel tracking tests in the Hamburg Wheel Tracking Test were performed by Koch Materials in Terre Haute, Indiana, on cores pulled from the pavement in 1993. Large diameter cores $(254 \mathrm{~mm}(10 \mathrm{in})$.$) were pulled from between the wheel paths in the high speed lane to avoid$ the effects of traffic consolidation.

The cores were mounted in aluminum frames with plaster of paris. After curing, the frames were placed in the test apparatus for loading. The samples were submerged in $50^{\circ} \mathrm{C}$ $\left(122^{\circ} \mathrm{F}\right)$ water and allowed to equilibrate before testing.

The Hamburg Wheel Tester uses a steel wheel that imparts a $705 \mathrm{~N}$ (158 lb.) normal force to the specimen, producing a contact stress of $1,480 \mathrm{kPa}(215 \mathrm{psi})$. The wheel reciprocates over a $23 \mathrm{~cm}$ (9 in.) track. Each pass takes approximately one second. Under load, the wheel presses into the surface of the mix. The wheel will indent the surface more for mixtures with low resistance to rutting than for more resistant mixes. Each sample is subjected to 20,000 passes of the wheel or is loaded until $20 \mathrm{~mm}(0.8 \mathrm{in})$ of deformation occurs. $(\underline{60})$

There are four parameters of interest measured in the test, as shown in Figure 36. One is post-compaction. Frequently during the first 1,000 passes of the wheel, the mix experiences consolidation under the wheel. A low consolidation level is preferred. After the rate of compaction stabilizes, permanent plastic deformation of the sample begins. This plastic deformation is related to rutting and is measured by the creep slope, in units of passes per millimeter of rut depth. The greater the slope, the more passes of the wheel it takes to produce a millimeter of rutting. The creep slope is an indication of rutting behavior in the absence of any stripping that might occur.

The onset of stripping in the mixture can be detected in two ways. First, the water in the test chamber becomes cloudy as fine aggregates are released from the mix. Also, the impression of the wheel into the mix accelerates as moisture damage develops. This increase in deformation can be quantified in two measurements. One is the stripping inflection point, which is considered 
an indication of the potential to strip. A graph of deformation versus number of passes will show a sudden change in the rate of deformation when stripping begins. The point at which this change occurs is the stripping inflection point. Higher stripping inflection points indicate mixtures that are more resistant to stripping.

The rate of deformation after the stripping inflection point is also an indicator of resistance to moisture damage. This is indicated by the stripping slope, expressed in passes per millimeter of rut. The stripping slope is considered an indication of the severity of stripping. Higher values of the stripping slope (more passes per millimeter) indicate less stripping damage.

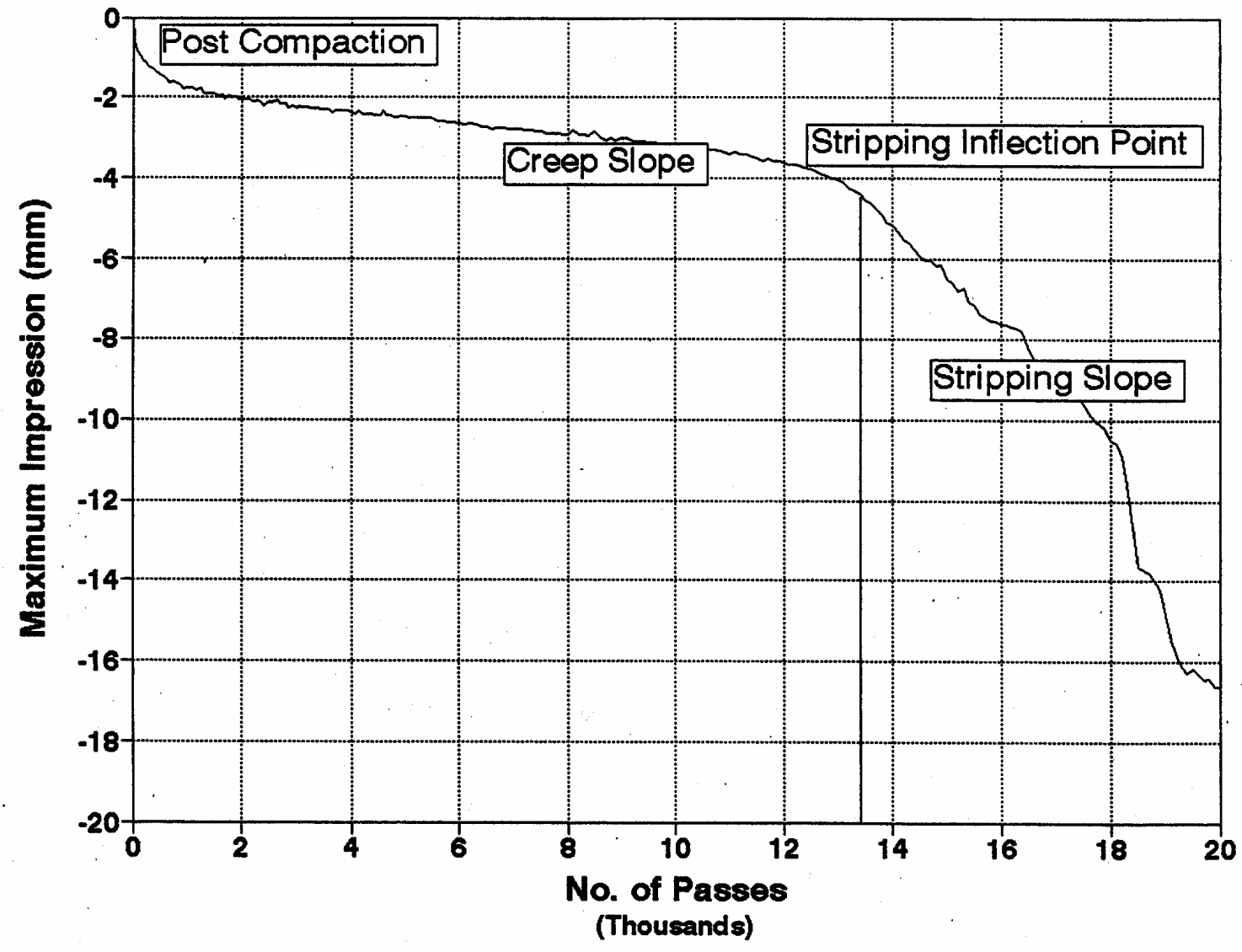

Figure 36 Generalized Wheel Tracking Parameters (from $\underline{60}$ )

The City of Hamburg, Germany, has the most experience with the test and has established specifications to limit rutting. Hamburg requires that mixtures exhibit less than $4 \mathrm{~mm}$ ( 0.16 in.) of rutting after 20,000 passes. Aschenbrener reported that this was found to be too severe for Colorado mixes and that an upper limit of $10 \mathrm{~mm}(0.4 \mathrm{in}$.$) is more reasonable. ( \underline{60})$ 
Results of the wheel tracking tests are shown in Figure 37 and Table 25 below (68).

From these values, the mixes were compared for consolidation, stripping and creep, as shown in Table 25. Results are presented for one or two cores from each section. Although two cores were pulled for each mix, in some cases one was damaged and could not be tested.

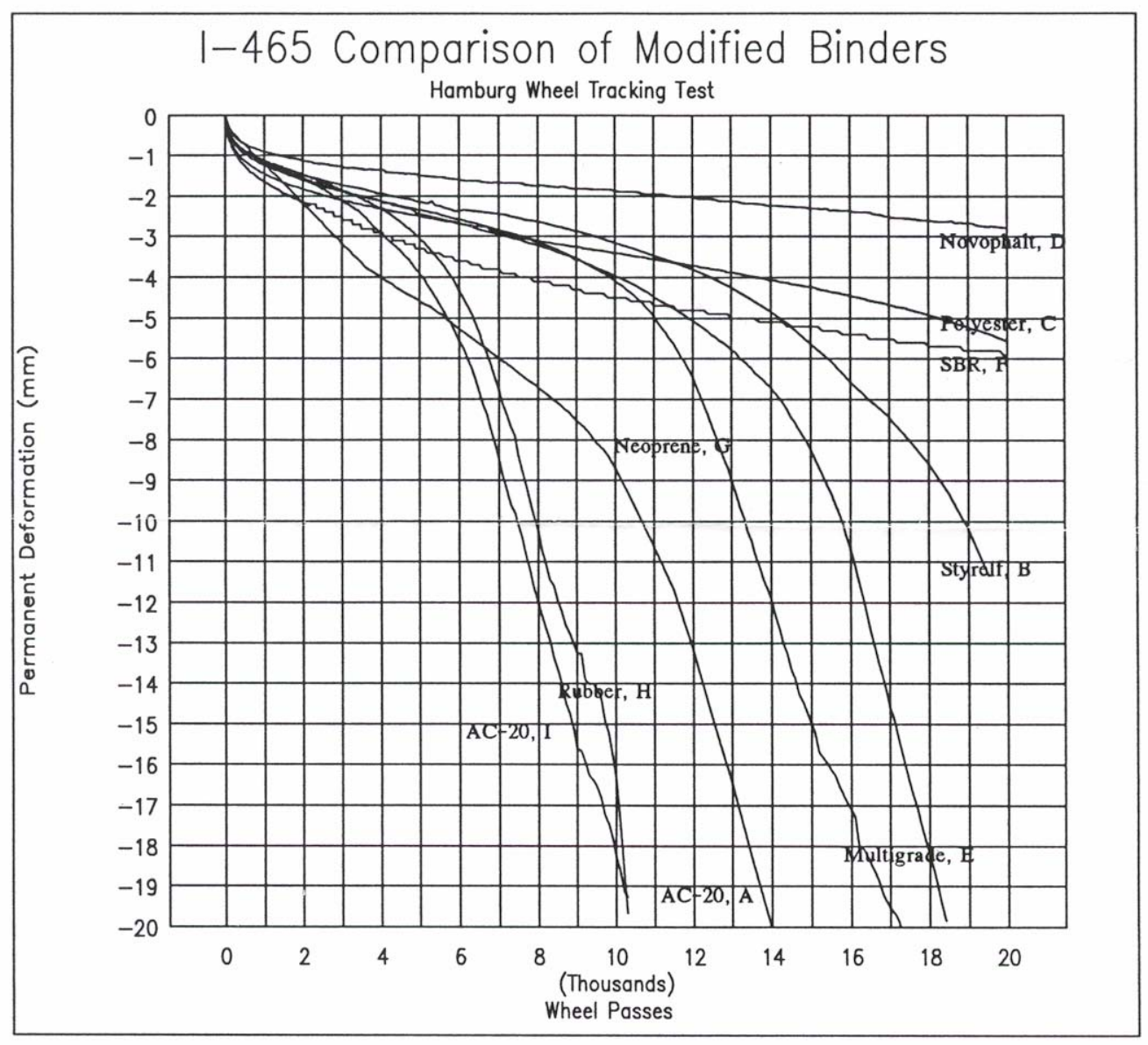

Figure 37 Wheel Tracking Results on Modified Mixes

The Control sections and the Asphalt Rubber were consistently among the worst performers by all four test parameters. The Novophalt (PE), fibers and SBR were among the best. The Novophalt was the only mix that would meet Hamburg's stringent requirement of $4 \mathrm{~mm}$ or less of rutting after 20,000 passes. The Fibers and SBR would also meet the more lenient requirement of $10 \mathrm{~mm}$ suggested by Colorado. (무)

High creep slopes indicate greater resistance to rutting in the absence of stripping, since it takes more wheel passes to produce $1 \mathrm{~mm}$ of deformation. The mixture with the greatest resistance to rutting by this measure was the Novophalt (PE). The SBR, Fibers and PAC had 
similar slopes, but they were only about half the value of the PE slope. The control sections and the Asphalt Rubber had the lowest creep slopes, indicating the greatest tendency to rut.

While the creep slope and stripping slope can be used to rank mixes relative to each other, there are no standards for limits on these values. The rut depth after 20,000 passes is the only standard applied. The SBR mixture showed no signs of stripping in the Hamburg device. The fiber mix showed very little tendency to strip and the MGAC, PAC and Novophalt mixtures showed similar resistance to stripping. The Control and Asphalt Rubber mixtures definitely showed the highest potential for stripping. 
Table 25 Wheel Tracking Test Data

\begin{tabular}{|l|c|c|c|c|c|c|c|c|c|}
\hline Section & $\mathrm{A}$ & $\mathrm{B}$ & $\mathrm{C}$ & $\mathrm{D}$ & $\mathrm{E}$ & $\mathrm{F}$ & $\mathrm{G}$ & $\mathrm{H}$ & $\mathrm{I}$ \\
\hline Binder & $\mathrm{AC}-20$ & $\mathrm{PAC}$ & Fibers & Novo & MGAC & SBR & Neo & AR & AC-20 \\
\hline Height, in. & 2.13 & 2.33 & 1.56 & 1.43 & 1.83 & 1.71 & 1.76 & 1.31 & 1.6 \\
(Core 1, Core 2) & 2.55 & 1.27 & 1.59 & 1.38 & & & 1.81 & 1.33 & 1.6 \\
\hline Post Compaction, mm & 0.6 & 1.2 & 1.6 & 1.2 & 1.0 & 2.1 & 1.2 & 0.7 & 0.6 \\
\hline Creep Slope, passes/mm & 1,175 & 5,570 & 5,335 & 13,815 & 3,655 & 5,950 & 4,350 & 2,605 & 1,990 \\
\hline Stripping Inflection Pt., & 11,000 & 13,645 & 17,760 & 13,035 & 14,485 & N/S & 10,530 & 5,830 & 5,335 \\
passes & & & & & & & & & \\
\hline Stripping Slope, passes/mm & 290 & 830 & 3,880 & 10,160 & 265 & N/S & 410 & 305 & 310 \\
\hline
\end{tabular}

Notes: Cores for Section A were both cracked on side.

Core B1 had large void on the bottom that was filled with plaster for testing.

Cores E1 and F2 were not tested due to breakage. 
Table 26 Summary of Wheel Tracking Performance Rankings

\begin{tabular}{|c|c|c|c|c|}
\hline Property & $\begin{array}{l}\text { Rutting } \\
\text { Resistance }\end{array}$ & $\begin{array}{l}\text { Stripping } \\
\text { Resistance }\end{array}$ & $\begin{array}{l}\text { Stripping } \\
\text { Severity }\end{array}$ & $\begin{array}{l}\text { Overall } \\
\text { Performance }\end{array}$ \\
\hline Indicator & Creep Slope & Inflection Pt. & Stripping Slope & \\
\hline \multirow[t]{8}{*}{ Best } & $\mathrm{PE}$ & SBR (0) & SBR (0) & $\mathrm{PE}$ \\
\hline & SBR & Fibers & $\mathrm{PE}$ & Fibers \\
\hline & PAC & MGAC & Fibers & SBR \\
\hline & Fibers & PAC & PAC & PAC \\
\hline & $\mathrm{Neo}$ & $\mathrm{PE}$ & Neo & MGAC \\
\hline & MGAC & Control (A) & Control (I) & $\mathrm{Neo}$ \\
\hline & $\mathrm{AR}$ & $\mathrm{Neo}$ & $\mathrm{AR}$ & Control (A) \\
\hline & Control (I) & AR & Control (A) & $\mathrm{AR}$ \\
\hline Worst & Control (A) & Control (I) & MGAC & Control (I) \\
\hline
\end{tabular}

Field performance of these mixtures indicated that there has been no significant rutting and there are no signs to date of stripping problems. Stripping can manifest itself in many ways, including raveling, rutting, depressions surrounding cracks, corrugations, and more. (드) Cores are the only way to definitively detect stripping. No stripping has been observed in any cores from I-465. The development of stripping requires the presence of moisture, high temperatures and high traffic loading. (요 $)$ High traffic levels and high temperatures have certainly been present on this pavement, but perhaps moisture has either not been there or it has not been in the pavement at the same time the high temperatures occurred. Crack sealing has been performed routinely on this project, which can help to prevent water from intruding into the pavement. In addition, the existing concrete pavement was undersealed prior to construction of the overlay. This may have limited the possibilities of water wicking up from below the pavement by sealing the interface between the subgrade and the concrete.

These results, then, indicate that most of the mixtures tested could undergo stripping if the conditions were conducive, but that stripping has not yet occurred, perhaps because the overlay has been protected from water intrusion. While these results indicate that some of the mixes are much more likely to rut than others, no significant rutting has been observed. 
Modification of the binder did improve both the rutting and stripping resistance in most cases (i.e. the control mixes performed the worst).

\subsubsection{Indirect Tensile Testing}

In the indirect tensile creep test (ITC), a $150 \mathrm{~mm}$ diameter by $50 \mathrm{~mm}$ high sample is loaded in static compression across a vertical diameter. The load is held constant while the horizontal and vertical deformations of the sample are recorded over a period of time (in this case, 240 seconds). Creep compliance is then calculated using the load and resulting displacement of the specimen as a function of time. The creep test is normally performed at three temperatures $\left(0,-10\right.$ and $\left.-20^{\circ} \mathrm{C}\right)$. Following ITC testing, indirect tensile strength (ITS) testing is performed at $-10^{\circ} \mathrm{C}$. ITS testing determines the fracture strength of a specimen by loading it at a constant deformation rate of $12.5 \mathrm{~mm} / \mathrm{min}$ until a fracture is formed. Specimen dimensions and peak load are then used to calculate the fracture strength. The test procedures used for ITC and ITS testing are described in more detail in AASHTO TP9, Standard Test Method for Determining the Creep Compliance and Strength of Hot Mix Asphalt (HMA) Using the Indirect Tensile Test Device.

Retained samples of four of the surface and intermediate courses were tested. These four sets of materials were selected based on the low temperature binder testing data presented in 6.1.2. As shown in Figure 30, the PAC, MGAC, PE and AC-20 exhibit different performance in terms of failure stress and strain. IDT testing was performed on mixtures with these four binders to determine if the mixture testing at low temperatures demonstrated the same behavior. Attempts were made to test the base mixtures with these binders as well, but difficulties in reaching the target air void content made this impossible. After repeated attempts to compact the specimens to $7 \pm 0.5 \%$ air voids, cracking of the aggregate began to appear.

The mixture samples had been retained in sealed paint cans in the laboratory since construction. It cannot be determined how much the samples aged, or if they aged differently, during this time. The base mixtures had been stored in open chicken buckets, which may help to explain the difficulties in compacting the specimens. The base materials may have hardened too much to compact properly when heated. Mixtures for IDT testing are typically long term oven aged to simulate service aging. These mixtures were not oven aged since they were over ten years old. It is unlikely, however, that these specimens had aged hardened as much as the pavement did over the same length of time. 
Table 27 Results of Indirect Tensile Testing

\begin{tabular}{|c|c|c|c|c|c|}
\hline \multirow{2}{*}{ Additive } & $\begin{array}{c}\text { Mean } \\
\text { Strength } \\
\mathrm{kPa}(\mathrm{psi})\end{array}$ & $\begin{array}{c}\text { Std. Deviation } \\
\mathrm{kPa}(\mathrm{psi})\end{array}$ & $\begin{array}{c}\text { C.V. } \\
\%\end{array}$ & $\begin{array}{c}\text { Mean A.V. } \\
\%\end{array}$ & $\begin{array}{c}\text { Estimated } \\
\text { Critical Temp. } \\
{ }^{\circ} \mathrm{C}\left({ }^{\circ} \mathrm{F}\right)\end{array}$ \\
\hline SURFACE LAYER & \multicolumn{5}{|l|}{} \\
\hline PAC & $2927(424)$ & $183(27)$ & 6.2 & 7.4 & $-30(-22)$ \\
\hline MGAC & $2631(382)$ & $165(24)$ & 6.3 & 7.0 & $-3(26)$ \\
\hline PE & $3152(457)$ & $104(15)$ & 3.3 & 6.9 & $-35(-31)$ \\
\hline AC-20 & $3208(465)$ & $81(12)$ & 2.5 & 7.1 & $-31(-23)$ \\
\hline INTERMEDIATE LAYER & & & & $-25(-13)$ \\
\hline PAC & $3291(477)$ & $531(77)$ & 16.1 & 7.5 & $-4(25)$ \\
\hline MGAC & $2228(323)$ & $216(31)$ & 9.7 & 7.6 & $-19(-2)$ \\
\hline PE & $2672(387)$ & $420(61)$ & 15.7 & 6.8 & $-25(-13)$ \\
\hline AC-20 & $3010(436)$ & $*$ & $*$ & 6.2 & \\
\hline
\end{tabular}

* Replicate sample broke while conducting creep compliance tests

Table 27 shows the mean strength of the specimens. This represents the average of two or three specimens, except in the case of the AC-20 intermediate mix. The replicate sample in this case broke during creep testing. The air void contents are all within $0.5 \%$ of the $7 \%$ target, except for the single AC-20 intermediate course sample, which had a low air void content.

The data was analyzed using a spreadsheet developed by Don Christensen called LTSTRESS. This spreadsheet uses the creep test data to calculate creep compliance as a function of time. The spreadsheet uses the creep and strength data to estimate the critical temperature at which the pavement may be expected to crack. The estimated critical cracking temperatures are shown in the last column of Table 27.

Figure 38 shows an example of the thermal stresses vs. temperature that are estimated from the creep data collected at $0,-10$ and $-20^{\circ} \mathrm{C}$. The tensile strength data is then used to determine the critical pavement temperature where cracking may occur. 


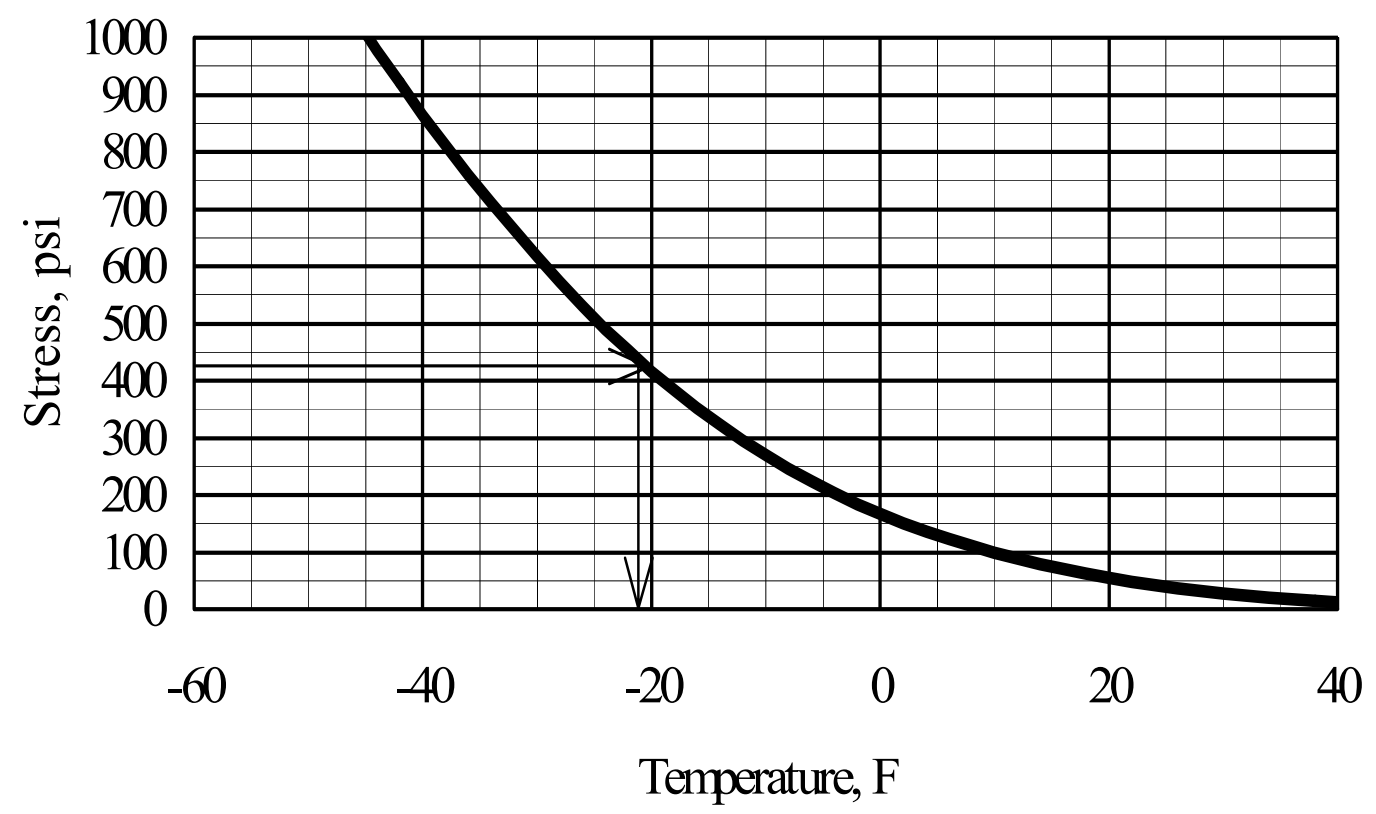

Figure 38 Estimated Thermal Stress and Strength of the PAC Surface

The data summarized in Table 27 shows that the PE, PAC and AC-20 mixtures have similar critical cracking temperatures and the MGAC section has significantly higher critical cracking temperatures. This would suggest that the MGAC section would crack earlier and crack more than the other sections. The field performance, however, does not substantiate this conclusion. In the field, the PE cracked much more extensively and at an earlier age than the other modified binders and also cracked more than the control mixtures. Also, the field performance shows a striking difference in the cracking resistance of the control (AC-20) and PAC sections, though their performance in this testing is similar.

The observed differences between the laboratory and field performance on the mixtures may be due to the state of aging of the mixtures. Perhaps the MGAC aged more in storage that the other mixtures. The PE mixtures may have age hardened more in the field than they did in the lab, producing more cracking. Exposure to the ultraviolet light of the sun may have accelerated the aging of the PE, for example. The correlation between lab and field performance could possibly be improved by using specific coefficients of thermal contraction for each binder. Since that data was not available, this analysis used the same default value for all of the binders. 


\section{INTERPRETATION AND ANALYSIS}

The field and laboratory results are analyzed and discussed in this chapter.

\subsection{Field Trial of Modifiers}

The field sections are all performing well in terms of rutting. No appreciable rutting has been measured on any of the sections, either during the manual distress surveys or by the Roadway Management system. Since the same aggregates and similar gradations were used for all of the mixtures, it is not surprising that the sections performed similarly. The fact that no rutting occurred on this project can be attributed to INDOT's revised Marshall mix design practices, coupled with high quality aggregates and close attention to detail during construction by the agency and contractor.

There are marked differences, however, between the various sections in terms of cracking. Cracking is widely recognized as a binder-related distress, so the different modifiers could reasonably be expected to perform differently. Some of the sections cracked extensively within three to six years after construction. Other sections are still performing well after 11 years. The best performers include the SBR, PAC and AR. A second tier of performance includes the Neoprene, Fibers and MGAC. The worst performers are the unmodified control sections and the Novophalt.

The observed cracking is not merely reflective cracking from the underlying portland cement concrete layer. The transverse crack spacing does not conform to the crack and joint spacing of the concrete section, nor to the locations of full depth concrete patches placed before overlaying. In addition, the worst performers exhibit extensive longitudinal cracking, which can not be caused by the underlying concrete. Much of the cracking is apparently caused by brittleness of the binder, especially in the PE section.

While the Asphalt Rubber is one of the better performers, the high cost of the material makes it hard to justify its use. The SBR and PAC performed nearly as well at a much lower price. Past and present performance of these three sections do not suggest that there is likely to be a great difference in the ultimate life of these sections. Other factors will probably lead to overlaying the entire contract within the next few years. It seems unlikely, therefore, that the asphalt rubber would last long enough to justify its much greater initial cost. 
No other significant distresses were noted during field surveys. There were no signs of stripping, raveling or other distress.

The findings of differences in the frictional performance of the modified sections versus the control sections were somewhat unexpected. In general, the modified sections did maintain their friction level longer and at a higher level than the control sections. Since the same aggregates were used in all of the sections, the difference is apparently due to a change in how well the binders retained the aggregates and macrotexture of the pavement. Some of the pavement sections, particularly the SBR, show an open texture that may be providing increased macrotexture and, therefore, higher friction values.

\subsubsection{Binder Testing}

The binder test results identified the polyethylene modifier as the most prone to cracking. The control binder (AC-20) was also identified as likely to crack. This lends credence to the recommended new procedures for testing modified binders. Conventional MP1 testing, however, also ranked the binders correctly in terms of cracking. With this limited testing, the MP1 tests appeared to be as accurate as the MP1a testing, which is much more time consuming. At this point and based on this limited data, there does not appear to be a compelling need to implement MP1a. That situation may change in the future as more data is collected and analyzed.

High temperature binder testing revealed that all of the modifiers stiffened the binder. High temperature binder stiffness relates to pavement rutting. As no rutting was observed in the field, no significant laboratory differences were expected. The aggregate framework and overall mix design have a greater influence on rutting than the binder.

\subsubsection{Mixture Testing}

Indirect tensile testing was performed on selected, retained samples of the modified and control mixtures. This testing showed that the MGAC mixtures might be expected to crack more than the other mixtures tested (PE, PAC and AC-20). The MGAC had substantially higher critical cracking temperatures than the other mixtures $\left(-3^{\circ} \mathrm{C}\right.$ vs. approximately -25 to $\left.-30^{\circ} \mathrm{C}\right)$. 
This does not correlate with the observed field performance, however. The PE cracked much more extensively in the field than the PAC and MGAC sections.

Hamburg wheel track testing of the mixtures showed that the Control and Asphalt Rubber sections have the greatest tendency to rut. The Polyethylene, SBR and Fibers performed the best in this test. No appreciable rutting has been observed on any of the sections in the field, however. The loaded wheel testing also indicated that the control and AR mixtures had the highest tendency to strip, but no signs of stripping have been observed.

\subsubsection{Relationship of Laboratory Results to Field Performance}

The new binder testing protocols were able to identify the test sections that exhibited the most cracking in the field. The PE and AC-20 had the lowest failure strains and stresses. The MGAC had intermediate failure stresses and strains. The PAC and Neoprene binders showed the highest failure strains and failure stresses. The observed cracking in the field followed a similar trend. The critical temperatures based on failure strain correlated best to the observed cracking, though the correlation based on failure stress was also good. Correlation with

The IDT test results indicated that the PE, PAC and AC sections would perform best in terms of cracking and the MGAC would perform the worst. This does not agree with the observed field performance, where the PE cracked much more than the PAC and MGAC sections. Differential aging of the laboratory specimens versus the pavement sections may have caused this discrepancy. Additional field validation of the mixture cracking estimates using the IDT is needed. The general consensus seems to be that the cracking models in Superpave work quite well, though they did not do so in this testing.

The Hamburg Wheel Tracking results did not correlate well to the observed field performance. These results showed that the control and asphalt rubber mixtures would be prone to rutting and stripping. No evidence of stripping and only minimal rutting has been observed in the field. This may be because the moisture necessary for stripping to occur is not present due to the internal drainage within the pavement structure. The strong aggregate framework in these mixtures may have prevented rutting. 


\section{CONCLUSIONS AND SUGGESTED RESEARCH}

This section summarizes the conclusions drawn from the data generated over 11 years of field service and laboratory testing.

1. Construction of the modified mixtures went smoothly for the most part. A variety of methods was used to introduce the modifiers or modified binders, and all were effective. Reacting the crumb rubber in the hot asphalt cement was time consuming and caused the most problems in terms of production. Laydown and placement were successfully accomplished with conventional equipment. From a construction point of view, then, most of the modifiers used were could be incorporated in future work fairly easily.

2. The field performance demonstrates that some of the additives performed much better in terms of cracking than others. Most performed better in terms of cracking than did the unmodified control sections. It is important to remember, however, that construction of these tests sections predates the implementation of PG binder grading and the binders do not all meet the same PG grade. They were modified according to the recommendations of the supplier and common usage at the time.

3. The newly developed tests for modified binders (MP1a) were able to identify the binders that exhibited the most cracking in the field. Implementation of these new tests should help to avoid using modified binders that are prone to cracking.

4. Conventional PG binder testing in the BBR, however, also ranked the modifiers correctly in terms of cracking performance. It appears the MP1 tests work sufficiently well to evaluate the particular modifiers used here, and it may not be necessary to implement MP1a to ensure improved cracking performance. This needs to be verified with additional information on other modifiers and other locations.

5. Indirect tensile testing of the mixtures was not as successful at identifying the mixtures that exhibited the most cracking in the field trial. This may have been due to differential aging of the mixtures in storage vs. field aging.

6. The field performance also demonstrated that binder modification is not necessary to control rutting. Properly designed and constructed asphalt mixtures can perform under heavy traffic without rutting, at least in Indiana. 
7. Somewhat surprisingly, the modified sections retained higher friction levels for a longer period of time than the unmodified control sections. This finding should be studied further to determine if it holds as a general rule.

8. No other distresses were noted in the field trials -- perhaps a testament to the care exercised by the contractor during construction.

\subsection{Overall Summary}

The use of modified binders did, for the most part, improve the field cracking performance over that of unmodified binders. The Novophalt binder, however, apparently increased the brittleness of the binder and mixture, leading to extensive cracking. The newly developed binder testing protocols, both MP1 and MP1a, were able to identify this binder as a potential problem. The other modifiers all improved the cracking resistance. The SBR, PAC and Asphalt Rubber sections exhibited the least cracking. The high cost of the rubber, however, may preclude its use.

The modified materials also exhibited slightly higher friction values in the field, perhaps by retaining macrotexture for a longer period of time. While friction alone may not be sufficient justification for the use of modified binders, it may be a side benefit.

Modification of the binder is not necessary to produce pavements that will not rut under heavy traffic. Even the control sections performed well here with only minimal rutting. Good aggregate gradations, proper mix designs and close attention to detail during construction contributed to the good rutting performance.

This study shows that modifiers can improve the cracking resistance of the mixtures in which they are used, provided the modified binders meet the PG grade appropriate for the location. It appears, based on this limited data, that the low temperature tests in MP1 using the BBR were able to rank the binders in terms of cracking resistance as well as the newly proposed tests under MP1a. Additional information is needed to ascertain whether INDOT should move to adopt MP1a, though based on this data, there does not seem to be a compelling reason to do so. 


\subsection{Suggested Research}

This research provided a unique opportunity to evaluate the link between field performance and laboratory properties. It was, however, limited in a number of respects. Some of the limitations include the following:

- $\quad$ The field trial was conducted in one location, which limits the environmental effects experienced by the modified materials.

- The experimental project was an overlay over portland cement concrete. This type of project was intentionally selected to provide a severe test of the modifiers' performance. This did, however, complicate the interpretation of the observed cracking. Because of the underlying concrete, the cracking is not merely thermal, but is no doubt at least partially reflective cracking.

- $\quad$ Only seven modifiers could be included in the study. As it was, the project was a logistical nightmare for the contractor.

- $\quad$ Because the SHRP specifications were under development during the early years of the field trial, samples of the materials used were held in storage until the protocols were finalized. Binder samples and some mixture samples were stored in sealed paint cans. Other mixture samples were stored in unsealed chicken buckets. The samples were somewhat protected from temperature fluctuations, but were not under ideal conditions for the entire storage period. The changes this extended storage time brought about in the materials cannot be conclusively determined.

- The amount of each sample available was limited, so there were tests that could not be conducted. For example, improved test methods for mixture fatigue cracking could have been used to investigate the relationship to the observed cracking.

Because of these limitations, there is a need for additional research. Some of the possible research topics include the following:

- Modification is widely used to stiffen binders at high temperatures to help control rutting. Due to the mix design and aggregates used in this research, however, none of the sections developed appreciable rutting. One potential research topic would be an investigation of the potential to reduce rutting by using a modified binder with less desirable aggregates, such as gravels, or a poorer aggregate gradation. 
- $\quad$ More modifiers need to be evaluated over a range of environmental and traffic conditions. Modifiers should be compared on the basis of equal PG grades.

- $\quad$ Additional field validation of the modified binder testing protocols and criteria as well as the mixture low temperature cracking predictions is also needed. 


\section{LIST OF REFERENCES}

1. Stroup-Gardiner, Mary and David E. Newcomb, "Polymer Literature Review," Minnesota Department of Transportation, Report No. MN/RC-95/27, St. Paul, MN, 1995, 220 pp.

2. King, Gayle, Helen King, R. D. Pavlovich, Amy L. Epps and Prithvi Kandhal, "Additives in Asphalt," Asphalt Paving Technology 1999, $75^{\text {th }}$ Historical Review, Journal of the Association of Asphalt Paving Technologists, Vol. 68A, 1999, pp. 32-69.

3. Romine, Robert A., Maghsoud Tahmoressi, R. David Rowlett and D. Fred Martinez, "Survey of State Highway Authorities and Asphalt Modifier Manufacturers on Performance of Asphalt Modifiers," Transportation Research Record, No. 1323, Transportation Research Board, Washington, DC, 1991, pp. 61-69.

4. Bahia, Hussain U., Dario Perdomo and Pamela Turner, "Applicability of Superpave Binder Testing Protocols to Modified Binders," Transportation Research Record, No. 1342, Transportation Research Board, Washington, D.C., 1992, pp. 16-23.

5. Button, Joe W., "Summary of Asphalt Additive Performance at Selected Sites," Transportation Research Record, No. 1342, Transportation Research Board, Washington, D.C., 1992, pp. 67-75.

6. Terrel, Ronald L., and Jon A. Epps, Using Additives and Modifiers in Hot Mix Asphalt (Part A), National Asphalt Pavement Association, Lanham, MD, Report No. QIP 114A, 1989.

7. Michael Heitzman, "Design and Construction of Asphalt Paving Materials with Crumb Rubber Modifier," Transportation Research Record, No. 1339, Transportation Research Board, Washington, D.C., 1992, pp. 1-8.

8. Hui, Joseph C. T., Geoffrey R. Morrison and Simon A. M. Hesp, "Improved LowTemperature Fracture Performance for Rubber-Modified Asphalt Binders," Transportation Research Record, No. 1436, Transportation Research Board, Washington, D.C., 1994, pp. 83-87.

9. Bahia, Hussain U., and Robert Davies, "Effect of Crumb Rubber Modifiers (CRM) on Performance Related Properties of Asphalt Binders," Asphalt Paving Technology 1994, Journal of the Association of Asphalt Paving Technologists, Vol. 63, 1994, St. Paul, Minnesota, pp. 414-449.

10. Kim, Sohee, Ssu-Wei Loh, Huachun Zhai and Hussain Bahia, "Advanced Characterization of Crumb Rubber Modified Asphalts Using Protocols Developed for Complex Binders," 
University of Wisconsin- Madison, Paper submitted to Transportation Research Board, Washington, DC, January 2001, preprint 25 pp. (Preprint CD-ROM)

11. Hanson, Douglas I., Kee Y. Foo, Elton Ray Brown and Robert Denson, "Evaluation and Characterization of a Rubber-Modified Hot Mix Asphalt Pavement," Transportation Research Record, No. 1436, Transportation Research Board, 1994, Washington, D. C., pp. 98-107.

12. Page, Gale C., "Florida's Initial Experience Utilizing Ground Tire Rubber in Asphalt Concrete Mixes," Asphalt Paving Technology 1992, Journal of the Association of Asphalt Paving Technologists, Vol. 61, 1992, St. Paul, Minnesota, pp. 446-472.

13. "For Roads That Go On Forever," Asphalt Materials, Inc., 1990, Indianapolis, Indiana.

14. Multigrade Asphalt Cement, Heritage Research Group Report, undated, Indianapolis, Indiana.

15. MAC in Indiana, Heritage Research Group, 1992, Indianapolis, Indiana.

16. Zhang, Xishun, and Gerald Huber, "Effect of Asphalt Binder on Pavement Performance: An Investigation Using the Superpave Mix Design System," Asphalt Paving Technology 1996, Journal of the Association of Asphalt Paving Technologists, St. Paul, Minnesota, Vol. 65, 1996, pp. 449-490.

17. Berkheimer, H. E., "Neoprene Modified Asphalt: The Material and Its Preparation," adapted from a presentation given before the International Symposium on the Use of Rubber in Asphalt Pavements, Salt Lake City, Utah, May 1971.

18. "Why More Polymers Are Coming for Asphalt," Highway \& Heavy Construction, June 1988, pp. 68-69.

19. Thompson, D.C., and J. F. Hagman, "The Modification of Asphalt with Neoprene," Proceedings of the Association of Asphalt Paving Technologists, Vol. 27, 1958, St. Paul, Minnesota, pp. 494-518.

20. "Stretch Your Paving Dollar with DuPont Asphalt Polymer Modifiers," DuPont Polymers, Wilmington, Delaware, 1992, 8 pp.

21. "Novophalt Permanent Deformation Laboratory Rutting Tester," Bulletin \#CT-004, Novophalt America, Inc., Sterling, Virginia, February 1989, 10 pages.

22. “Novophalt Rutting Lab Evaluation,” Bulletin \#CT-005, Novophalt America, Inc., Sterling, Virginia, February 1989, 7 pages. 
23. "Novophalt Rutting Evaluation Project Record," Bulletin \#PR-002, Novophalt America, Inc., Sterling, Virginia, February 1989, 22 pages.

24. "Novophalt Product Information Bulletin," Bulletin \#PIB-02, Novophalt America, Inc., Sterling, Virginia, February 1989, 11 pages.

25. “Novophalt User Guidelines,” Bulletin \#PIB-01, Novophalt America, Inc., Sterling, Virginia, February 1989, 3 pages.

26. “Denver's New E-470 Tests Low Density Polyethylene Modifier," Roads and Bridges, Sept 1991, p. 47.

27. “Asphalt Material Garners Top Honors in PN Contest,” Plastic News, Sept. 17, 1990, p. 1.

28. Jew, P., and R. T. Woodhams, "Polyethylene-Modified Bitumens for Paving Applications," Asphalt Paving Technology 1986, Journal of the Association of Asphalt Paving Technologists, St. Paul, Minnesota, Vol. 55, 1986.

29. Lee, Nolan K., and Simon A. M. Hesp, "Low Temperature Fracture Toughness of Polyethylene-Modified Asphalt Binders," Transportation Research Record, No. 1436, Transportation Research Board, Washington, D.C., 1994, pp. 54-59.

30. Little, Dallas N., "Performance Assessment of Binder-Rich Polyethylene-Modified Asphalt Concrete Mixtures (Novophalt)," Transportation Research Record, No. 1317, Transportation Research Board, Washington, D.C., 1991, pp. 1-9.

31. Button, Joe W., and Jon A. Epps, "Mechanical Characterization of Fiber-Reinforced Bituminous Concrete,” Report 4061-1, Texas Transportation Institute, College Station, Texas, February 1981, 99 pp.

32. Galinsky, J. D., "Performance of Bituminous Surface with Fibers on I-65, Final Report," Indiana Department of Highways, West Lafayette, Indiana, March 1984, 13 pp.

33. McDaniel, Rebecca S., "Supplemental Report: 1985 Update on Performance of Bituminous Surface with Fibers," Indiana Department of Highways, West Lafayette, Indiana, December 1985, 7 pp.

34. Jiang, Yi, and Rebecca S. McDaniel, "Application of Cracking and Seating and Use of Fibers to Control Reflective Cracking," Transportation Research Record, No. 1388, Transportation Research Board, Washington, D.C., 1993, pp. 150-159. 
35. "Pavement Condition Evaluation: Modified Asphalt Concrete, Livingston Avenue," Report RI \#89-0018, Resource International, Inc., Westerville, Ohio, June 1989, 86 pp.

36. Mills, David R., and Thomas Keller, Jr., "The Effectiveness of Synthetic Fiber-Reinforced Asphaltic Concrete Overlays in Delaware," Delaware Department of Transportation, Dover, Delaware, November 1982, 46 pp.

37. Scherocman, James A., "The Properties of Asphalt Concrete Mixtures Incorporating Polyester and Polypropylene Fibers," Report Prepared for GFC Materials Company and Hoechst Celanese Corporation, by James Scherocman, Consulting Engineer, Cincinnati, Ohio, February 1994, 12 pp.

38. Jenq, Yeou-Shang, and Pei Liu, "Performance Evaluation of Fiber Reinforced Asphalt Concrete," Report No. FHWA/OH-94/018, Ohio State University, Columbus, Ohio, 145 pp.

39. "Styrelf: The Asphalt for Today's Hot Mix," product literature circa 1985

40. Khosla, N. Paul, "Effect of Use of Modifiers on Performance of Asphalt Pavements," Transportation Research Record, No. 1317, Transportation Research Board, Washington, D.C., 1991, pp. 10-22.

41. Estakhri, Cindy K., Joe W. Button, "Evaluation of Styrelf-13 in Hot Mixed Asphalt Concrete," Research Report 0355, Texas Transportation Institute, College Station, Texas, May 1988, 76 pp.

42. Puzinausksas, V. P., E. T. Harrigan, "Modification of Asphalt Cement and Paving Mixes with Styrene and Butadiene Elastomer - Styrelf System," The Asphalt Institute, College Park, Maryland, September 1987, 49 pp.

43. Monismith, C. L., and A. A. Tayebali, "Behavior of Mixes Containing Conventional and Polymer (Styrelf) Modified Asphalts," University of California at Berkeley, Berkeley, California, February 1988.

44. “Ultrapave SBR Latex Polymers," Product Lit, no date, circa 1995.

45. Collins, J. H., M. G. Bouldin, R. Gelles and A. Berker, "Improved Performance of Paving Asphalts by Polymer Modification," Asphalt Paving Technology 1991, Journal of the Association of Asphalt Paving Technologists, Vol. 60, 1991, St. Paul, Minnesota, pp. 4379.

46. Button, J. W. and D. N. Little, "Asphalt Additives for Increased Pavement Flexibility," Texas Transportation Institute: Research Report 471-2F. 
47. Lee, D. Y., and T. Demirel, "Beneficial Effects of Selected Additives on Asphalt Cement Mixes," Iowa Department of Transportation, Ames, Iowa, August 1987.

48. King, William M., and Roland J. Doucet, Jr., "Latex Modified Asphalt and Experimental Joint Treatments on Asphaltic Concrete Overlays, Experimental Project No. 3 - Asphalt Additives," Report No. FHWA/LA-91-237, Louisiana Transportation Research Center, Baton Rouge, LA, June 1991, 70 pp.

49. Correspondence from Fred F. Frecker, President/Executive Director, Flexible Pavements, Inc. Columbus, Ohio, August 12, 1996.

50. Anderson, David A., and Thomas W. Kennedy, "Development of the SHRP Binder Specification," Asphalt Paving Technology 1993, Journal of the Association of Asphalt Paving Technologists, Vol. 62, 1993, St. Paul, Minnesota, pp. 481-507.

51. King, Gayle N., Helen W. King, Otto Harders, Pierre Chavenot and Jean-Pascal Planche, "Influence of Asphalt Grade and Polymer Concentration on the High Temperature Performance of Polymer Modified Asphalt," Asphalt Paving Technology 1992, Journal of the Association of Asphalt Paving Technologists, Vol. 61, 1992, St. Paul, Minnesota, pp. $29-66$.

52. King, Gayle N., Helen W. King, Otto Harders, Wolfgang Arand and Jean-Pascal Planche, "Influence of Asphalt Grade and Polymer Concentration on the Low Temperature Performance of Polymer Modified Asphalt," Asphalt Paving Technology 1993, Journal of the Association of Asphalt Paving Technologists, Vol. 62, 1993, St. Paul, Minnesota, pp. 122.

53. Tayebali, Akhtarhusein A., Bijal B. Vyas and Glen A. Malpass, "Effect of Crumb Rubber Particle Size and Concentration on Performance Grading of Rubber Modified Asphalt Binders," Progress of Superpave (Superior Performing Asphalt Pavement): Evaluation and Implementation, edited by Robert N. Jester, STP 1322, American Society for Testing and Materials, West Conshohocken, Pennsylvania, pp. 30-47.

54. Dongré, Raj, Joe W. Button, Robert Q. Kluttz and David A. Anderson, "Evaluation of Superpave Binder Specification with Performance of Polymer-Modified Asphalt Pavements," Progress of Superpave (Superior Performing Asphalt Pavement): Evaluation and Implementation, edited by Robert N. Jester, STP 1322, American Society for Testing and Materials, West Conshohocken, Pennsylvania, pp. 80-100.

55. Bahia, Hussain U., Walter P. Hislop, Huachun Zhai and Andres Rangel, "Classification of Asphalt Binders in Simple and Complex Binders," Asphalt Paving Technology 1998, Journal of the Association of Asphalt Paving Technologists, Vol. 67, 1998, St. Paul, Minnesota, pp. 1-41. 
56. Zeng, Menglan, Hussain U. Bahia, Huachun Zhai, Michael R. Anderson and Pamela Turner, "Rheological Modeling of Modified Asphalt Binders and Mixtures," Asphalt Paving Technology 2001, Journal of the Association of Asphalt Paving Technologists, Vol. 70, 2001, St. Paul, Minnesota, 40 pp.

57. Bahia, Hussain U., Huachun Zhai, Menglan Zeng, Yu Hu and Pamela Turner, "Development of Binder Specification Parameters Based on Characterization of Damage Behavior," Asphalt Paving Technology 2001, Journal of the Association of Asphalt Paving Technologists, Vol. 70, 2001, St. Paul, Minnesota, 25 pp.

58. Blankenship, Phillip B., Allen H. Myers, Andrea S. Clifford, Todd W. Thomas, Helen W. King and Gayle N. King, "Are All PG 70-22s the Same? Lab Tests on KY I-64 Field Samples," Asphalt Paving Technology 1998, Journal of the Association of Asphalt Paving Technologists, Vol. 67, 1998, St. Paul, Minnesota, pp. 493-552.

59. Stuart, Kevin D., and Walaa S. Magower, "Validation of Asphalt Binder and Mixture Tests that Predict Rutting Susceptibility Using the FHWA ALF," Asphalt Paving Technology 1997, Journal of the Association of Asphalt Paving Technologists, Vol. 66, 1997, St. Paul, Minnesota, pp. 109-152.

60. Aschenbrener, Tim, "Evaluation of the Hamburg Wheel-Tracking Device to Predict Moisture Damage in Hot Mix Asphalt," Transportation Research Record, No. 1492, Transportation Research Board, Washington, D.C., 1995, pp. 192-201.

61. E. R. Brown, Frazier Parker, Jr., and Michael R. Smith, "Study of the Effectiveness of Styrene Butadiene Rubber Latex in Hot Mix Asphalt Mixes," Transportation Research Record, No. 1342, Transportation Research Board, Washington, D.C., 1992, pp. 85-91.

62. Arthur M. Rucker, "Evaluation of Polymer Modified Bituminous Mixtures on US-41 in Terre Haute," Indiana Department of Transportation, Division of Materials and Tests, Indianapolis, IN, November 1990, 20 pp.

63. Letter to R. S. McDaniel from Arthur M. Rucker, November 27, 1990, Re: R-16911, SR 67, Neoprene Latex Modified Binder.

64. LTPPBind, Version 2.1, Federal Highway Administration, Washington, DC, 1999.

65. Climatological Data Annual Summary, Indiana 1992, Volume 97, Number 13, National Oceanic and Atmospheric Administration, Asheville, NC, 1992.

66. Majidzadeh, Kamran, and V. Kumar, Manual of Operation and Use of Dynaflect for Pavement Evaluation, Report No. FHWA/OH-83/004, Federal Highway Administration, Washington, D.C., 1983. 
67. Klemens, Thomas L., "DOT Puts Modifiers to the Test," Highway and Heavy Construction, January 1991, Vol. 134, No. 1, pp. 30-32.

68. Thomas, Todd W., "Wheel Tracking Report," Koch Materials Laboratory, Terre Haute, Indiana, September 11, 1993, 4 pp.

69. Roberts, Freddy L., Prithvi S. Kandhal, E. Ray Brown, Dah-Yinn Lee and Thomas W. Kennedy, Hot Mix Asphalt Materials Mixture Design and Construction, NAPA Education Foundation, Lanham, Maryland, 1996, pp. 70-71, 510-517.

70. Bahia, Hussain U., D. I., Hanson, M. Zeng, H. Zai, M.A. Khatri, R. M. Anderson, Characterization of Modified Asphalt Binders in Superpave Mix Design, NCHRP Report 459, Transportation Research Board, Washington, D.C., 2001. 


\section{Appendix A LITERATURE REVIEW}

There is a massive amount of literature concerning modified binders. The literature review presented here is not intended to be a comprehensive review of the entire body of literature, but does provide general background information, highlight the specific modifiers studied in this research, and relate testing and field performance of the subject modifiers. For more detailed information on polymer chemistry and modified binders, see the detailed literature review compiled by Stroup-Gardiner and Newcomb (1).

\section{A.1 Asphalt Additives and Modifiers}

Asphalt has been used since early recorded times, and patents for asphalt modification were granted as early as the 1840 's. Modification with polymers began to increase in the United States in the 1950's and has increased markedly over the last decade or two. (Polymer modification in Europe predated US implementation, beginning in the 1930's.) (2) Of 45 state highway agencies responding to a 1991 survey regarding experiences with modified asphalts, $86 \%$ reported using some form of polymer modified binder; $77 \%$ used anti-stripping agents; and $59 \%$ used fillers, fibers or extenders. ( $\underline{3})$

There are many applications for modifiers and a wide variety of types providing different benefits. The following discussion summarizes the impacts of modification in general terms then examines the use of selected modifiers of interest to this research.

\section{A.1.1 Impacts of Use of Modifiers}

King et al. summarized fourteen reasons to modify asphalts, based on a list developed by the National Center for Asphalt Technology.

"Asphalts have been modified to:

- stiffen binders and mixtures at high temperatures to minimize rutting and reduce the detrimental effects of load induced moisture damage,

- soften binders at low temperatures to improve relaxation properties and strain tolerance, thus minimizing non-load associated thermal cracking,

- improve fatigue resistance, particularly in environments where higher strains are imposed on the asphalt concrete mixture, 
- improve asphalt-aggregate bonding to reduce stripping,

- reduce raveling by improving abrasion resistance,

- minimize tender mixes, draindown, or segregation during construction,

- rejuvenate aged asphalt binders,

- replace asphalt cement as an extender,

- permit thicker films of asphalt on open-graded aggregates for increased durability,

- reduce flushing or bleeding,

- improve resistance to aging or oxidation,

- stiffen hot mix asphalt (HMA) layers to reduce required structural thickness,

- improve pavement durability with an accompanying net reduction in life cycle costs,

- replace PCC with asphalt construction methods that reduce lane closure times and user delay costs, and

- improve overall performance as viewed by the highway user." (2)

Bahia et al. noted that different classes of modifiers can bring about improvements in terms of different distresses in the resulting binders or mixtures. (The classes of modifiers are discussed in section A.1.2.) They summarized the effects of different types of modifiers on permanent deformation (rutting), fatigue cracking, low temperature cracking, moisture damage and oxidative aging. While there are differences between individual modifiers within a group, in general, the groups have fairly consistent effects on the five distress types. (4) These common effects will be discussed individually below.

Button offered a different perspective. In addition to summarizing the purported benefits of various modifiers, he pointed out some of the potential disadvantages of their use. Key among these is the cost of additional heating that is typically required to offset the higher viscosity of the modified binders. Hot storage of the binders over prolonged periods of time can cause thermal degradation of the modifier, reducing or eliminating the benefits and possibly causing other problems, like tenderness during construction. Compatibility of the modifier with the base asphalt is a common issue and often must be verified on a case-by-case basis. Quality control testing can be more complicated with modified binders. For example, conventional extraction tests may not be accurate for some modified binders in terms of binder content or recovered binder properties. Determining the modifier content in a given binder can be complex and expensive. (ㅁ) 


\section{A.1.2 Types of Modifiers}

Under the sponsorship of the National Asphalt Pavement Association, Terrel and Epps classified asphalt additives or modifiers into ten generic groups. These groups are shown in Table 1. Terrel and Epps provided descriptions of each group. (ㅁ) The modifiers evaluated in this research classify as rubbers, plastics and fibers (Groups 3, 4 and 6). Both rubbers and plastics are polymers, large chain molecules composed of repeating units called monomers. Fibers may also be composed of polymers, or they may be natural organic or mineral fibers. Fibers are characterized by their filament shape. Materials in Groups 3, 4 and 6 can act to reduce rutting and cracking. Modifiers in the other groups typically have other applications, such as reducing environmental damage (from moisture or aging), filling voids or partially replacing asphalt cement, among other uses.

According to Terrel and Epps, the rubber group includes materials that are elastic at room temperature. They are sometimes called elastomers because of that behavior. These materials may include natural or synthetic rubbers, finely divided recycled tire rubber or block copolymers. The rubber group can be further subdivided. Subdivisions include homopolymers, copolymers and terpolymers. Homopolymers are long chains composed of only one type of monomer; examples of homopolymers include natural rubber, poly-butadiene, polyisoprene and polychloroprene (Neoprene). Random copolymers, such as styrene-butadiene rubber (SBR), consist of two different monomers in a random pattern. Block copolymers are composed of repeating polymer blocks. Examples include styrene butadiene diblock (SB), styrene butadiene styrene (SBS), SIS (styrene isoprene styrene) and acrylonitrile butadiene styrene (ABS). (ㅁ) Terpolymers are composed of three repeating monomers, like ethylene propylene diene monomer (EPDM).

Many of these materials are thermoplastic, meaning they become more plastic when heated then return to their original state upon cooling. Their purpose for use in asphalt typically is to improve the temperature susceptibility of the base asphalt or to enhance its elasticity. ( $\underline{6}$ )

The plastic group includes polymers that can flow under load. Typically the polymers used in hot mix asphalt are thermosetting polymers; that is, they flow under load when heated, but "set" when cooled and will not soften again when reheated. Reheating may damage or destroy the structure, but it will not melt. (ㅁ) 
The fiber group includes short, very fine natural, synthetic or steel fibers. These fibers provide reinforcement and a high surface area, which allow the use of thicker binder films on the aggregate. (ㅁ) 


\section{A.2 Specific Additives}

The following sections summarize the pertinent literature related to the specific modifiers used on this project.

\section{A.2.1 Asphalt Rubber}

Asphalt rubber is a blend of conventional asphalt cement with ground tire rubber. The term "reclaimed rubber" refers to raw or unprocessed tire rubber, while "recycled rubber" refers to processed tire rubber. The asphalt cement is heated to high temperatures and the rubber is added. Asphalt rubber is supposed to improve flexibility and rutting resistance. (ㅁ)

There are several different processes for using rubber in asphalt, and conflicting terminology has been used in the past. Heitzman attempted to clarify and standardize the terminology. There are two basic processes using crumb rubber in asphalt pavements; the socalled wet and dry processes. In the wet process, finely divided rubber is added to hot asphalt cement, producing a modified binder called asphalt rubber. Two technologies are used to accomplish this blending, the McDonald and the continuous blending technologies. The McDonald technology, used in this study, is sometimes also called the Sahuaro or Arizona technology. The other process for using crumb rubber, the dry process, involves mixing rubber particles with the aggregate before incorporating the liquid asphalt. The resulting product is termed rubber modified hot mix asphalt. PlusRide is one common trade name for this product.

Due to the presence of carbon black and antioxidants in the rubber to prolong tire life, the aging of the blended binder is also reduced, which should result in improved pavement life. Elasticity, softening point, fracture temperature and cohesive strength are all purported to improve over unmodified binder. (ㅁ)

In the wet process, the ground tire rubber is blended with hot asphalt cement and a reaction occurs. Aromatic oils from the asphalt are absorbed into the rubber, causing the rubber to soften and swell. The rubber does not dissolve or melt in the asphalt. The rate of the reaction depends on the temperature, size of the rubber particles, aromatic nature of the asphalt and other factors. More finely ground rubber, with its higher surface area, reacts more quickly. (ㄱ) A mixing unit is needed to meter the rubber into the asphalt cement, agitate it and maintain the blend at elevated temperature to allow the asphalt-rubber reaction to occur. 
Construction of asphalt rubber pavements is typically the same as with conventional pavements, though higher mixing and compaction temperatures may be needed to offset the higher binder viscosity. Rubber-tired rollers are generally not used since the modified binder tends to stick to the tires. Costs can be $50-100 \%$ higher than conventional mixtures. (ㄱ)

Hui, Morrison and Hesp compared the low-temperature fracture toughness of various types and sizes of ground tire rubber with and without modification of the surface of the rubber particle. Modification with liquid polybutadiene (LPBD) and sulfur was done to enhance the reaction between the rubber and asphalt, strengthening the interface. Finer rubber particles (30-, 40- and 80-mesh) were found to provide greater increases in fracture toughness than coarser particles (10- and 20-mesh). Interfacial modification did increase the fracture toughness of all of the particle sizes. The authors point out that these test results need to be verified on mixtures and in the field. ( $\underline{8})$

Bahia and Davies evaluated the performance of binders incorporating three different types of ground tire rubber using the original Superpave binder test protocols in 1994, before Dr. Bahia embarked on a massive research project to develop test methods for modified binders, discussed in 2.4.1. They tested four asphalt cements modified with ambient shredded, cryogenically ground and extruded rubber. The resulting binders were tested in the rotational viscometer, dynamic shear rheometer, bending beam rheometer and direct tension tester. The thin film oven test and pressure aging vessel were used to condition samples prior to testing. Overall, Bahia and Davies found that they could use the Superpave binder testing protocols for testing CRM binders, although they cautioned that sample preparation is more difficult and that the tests were not developed to be used with CRM. Viscosity was greatly increased by the addition of rubber, which would complicate pumping and mixing at the plant. The CRM improved the rutting resistance of the binder as measured by $\mathrm{G}^{*} / \mathrm{sin} \delta$ mainly by increasing the modulus. The effect of CRM on the fatigue parameter $\left(\mathrm{G}^{*} \sin \delta\right)$ varied depending on the test temperature. While CRM had only a marginal effect on the bending beam rheometer properties, it did improve the strain at failure as measured in direct tension, though not to a great extent. The magnitudes of changes, in many cases, varied depending on asphalt and rubber source. The addition of CRM to the binders tested did indicate an improvement in the aging characteristics of the blended binders, as previously suggested. Several people commented during the discussion period on problems inherent in testing particulate materials in the Superpave protocols. (9)

Kim et al. used new test protocols developed under NCHRP 9-10, which are described in detail later, to evaluate crumb rubber modified binders. They tested three sizes of crumb rubber 
added to two different binders (PG 70-22 and PG 64-22) at two different rubber contents. They also looked at two binders produced under a patented process using reacted crumb rubber. The findings indicate that the concentration and size of the rubber particles used have a significant effect on the binder viscosity and on fatigue and strain dependencies, which are also strongly influenced by binder type. Direct tension testing showed that as rubber size and concentration increased, the failure stress and strain decreased. Size and concentration were not, however, important considerations for storage stability (the LAST test). Agitation was needed to reduce separation of the rubber particles from the asphalt binder. (10)

Hanson et al. evaluated the comparative field performance of conventional and rubbermodified hot mix and related performance to laboratory testing. The rubber-modified hot mix (RMHMA) they evaluated used very finely divided rubber (80-mesh). Laboratory properties measured included resilient modulus, indirect tensile strength, densification properties, stripping resistance and confined dynamic creep. The test results indicated the RMHMA had improved stripping resistance, resilient modulus and resistance to rutting compared to the unmodified control mix. (While the tensile strength ratio of the RMHMA was higher than the control, it should be noted it was still only $50 \%$ versus $38 \%$ for the control, which may indicate the mix was prone to stripping with or without the rubber.) There was no effect on tensile strength. Field performance after two years showed no cracking and only minimal rutting. (11)

The Florida DOT has also evaluated the performance of mixes incorporating finely ground tire rubber ( 80 mesh) following state legislation requiring FDOT to develop uses for reclaimed tire rubber. Page reported on the early performance of three test sections that used small percentages of rubber (5 to $12 \%$ ) in fine graded and open-graded surfaces. The percentages of rubber used were lower than those typically used in asphalt rubber, so the binder is referred to in Florida as rubber modified asphalt. The field trials showed that rubber modified mixtures could be constructed with minimal changes in operations. Early performance (two years or less) was acceptable, but long-term history was lacking at the time of the report. The addition of rubber allowed a higher asphalt content to be used in the open-graded mixes, leading to the expectation of increased durability for those mixes. Increased costs of at least $10 \%$ are expected, but may be offset by additional life. (12) 


\section{A.2.2 Multigrade Asphalt Cement (MGAC)}

Multigrade asphalt cement is a bituminous product developed at Heritage Research Group following their research into why some older bituminous pavements performed better than pavements constructed in the 1980's. (13) Their findings indicated that mixes with large aggregates, a strong aggregate framework and thick binder films were stronger and more durable than other mixtures. They then developed multigrade asphalt cement to ensure thick asphalt film coatings could be produced.

Multigrade asphalt cement is supposed to perform analogously to multigrade lubricating oil, like the 10W40 motor oil typically used in automobile engines. MGAC is purported to act like a hard asphalt at high temperatures and like a soft asphalt at low temperatures. It is expected to help maintain thick film thicknesses without draindown of the binder, increase mix stability at high temperatures and remain flexible at low temperatures. (13) Resistance to age hardening is another claimed benefit of MGAC. (14) MGAC is a non-newtonian gelled asphalt. (15)

Little has been reported in the literature on MGAC or its performance. One paper that has been published by Zhang and Huber reports on a comparison of the permanent deformation resistance of mixtures with various binder types. In addition to gelled asphalt (MGAC), Zhang and Huber evaluated mixes made with AC-20, polymer modified (SBR at two addition rates) and air oxidized binders, and with polyester fiber reinforcement. The Superpave Shear Tester was used to evaluate the mixtures' resistance to rutting. Their work showed that mixtures with gelled and polymer-modified binders could be expected to perform better than the other modified mixes. $(\underline{16})$

\section{A.2.3 Neoprene}

Neoprene is a synthetic rubber material added to asphalt emulsions or cements in latex form. The latex consists of neoprene particles about 0.2 microns in diameter dispersed in water. The typical solids content is approximately $45-60 \%$. Neoprene is typically added to the asphalt at a rate of $4-6 \%$ wet weight $(2-3 \%$ dry weight $)$. (ㅁ$)$

Neoprene reportedly improves the temperature sensitivity of the base asphalt, the high temperature viscosity, durability (as measured by toughness and tenacity before and after TFOT), elasticity and chip retention for chip seals. The compatibility of the neoprene with the base asphalt must be verified by pretesting by the manufacturer. $(\underline{6}, \underline{17})$ Neoprene is tough and 
rubbery, with a tensile strength of about 4000 psi and $900 \%$ elongation. DuPont says neoprene acts like a "mechanical adhesive," sticking to both aggregate and asphalt without chemically bonding. (18)

Neoprene is the oldest commercial synthetic rubber and has been used in asphalt cement since the 1950's. As early as 1958, neoprene modified asphalt was the subject of a paper at AAPT. (19)

In that paper, Thompson and Hagman reported on binder testing of 85-100 penetration grade asphalts modified with the addition of neoprene latex. The tests they used included penetration, softening point, ductility, flow, torsional recovery, and toughness and tenacity. They found that "neoprene alters the softening point, flow resistance, tenacity and toughness of the asphalt to give a less temperature-sensitive, tougher product." Low temperature $\left(39.2^{\circ} \mathrm{F}\right.$ or $\left.4^{\circ} \mathrm{C}\right)$ ductility of the asphalt was doubled by the addition of only $1 \%$ neoprene and increased even further at higher addition rates. Neoprene also reduced the age hardening of asphalt subjected to the thin film oven aging procedure. Thompson and Hagman theorized that neoprene retained more of the asphalt's volatile compounds, thus reducing the age hardening effect. An addition rate of between 1 and 5\% neoprene, by weight of asphalt, is optimal and $3 \%$ is commonly used. At concentrations above $7 \%$ neoprene, the blend becomes unstable and prone to gelling or separation of the neoprene from the asphalt. (19)

Neoprene has reportedly doubled the life of hot mix asphalt in some severe applications, such as on the Rock Island Centennial Bridge between Illinois and Iowa. Neoprene-modified asphalt performed for 12 years on that bridge, which carried eight million vehicles per year. Unmodified asphalt previously used on the same bridge purportedly had to be replaced every five years. $(\underline{20})$

\section{A.2.4 Novophalt}

Novophalt is a polymer modified asphalt cement developed in Europe in 1976. It was introduced in the United States ten years later. Novophalt reputedly stops rutting and shoving at elevated temperatures, and increases resilience and durability. The process also is reported to increase cohesion and adhesion of the binder to the aggregate, thereby reducing stripping and raveling. A 50 to $100 \%$ increase in service life is claimed. (21) The manufacturer cites wheel tracking test results $(\underline{21}, \underline{22})$ and field performance $(\underline{23})$ to support this claim. One set of results indicates that at $60^{\circ} \mathrm{C}$, a mixture with Novophalt was seven times more resistant to rutting than 
the control. Low temperature cracking can be controlled, the manufacturer indicates, by using a low viscosity base asphalt. (느)

Novophalt is produced by adding about 4 to $6 \%$ (by weight of binder) low density polyethylene (LDPE) to asphalt cement in a high shear mixer. Recycled polyolefins can be used in the process. (25) Milk jugs typically contain this type of plastic, as do plastic trash bags and sandwich bags. (26) Post-consumer and post-industrial recycled polyethylene can both be used. In 1990, about $92 \%$ of the resin used by Novophalt was recycled material. (27)

A mobile blending unit is provided that can be set up at the hot mix plant to pre-blend the polyolefin pellets into the asphalt cement. The mobile unit contains a heating system, high shear colloid mill and agitation. Following blending, mix production and construction are accomplished conventionally. A mixing temperature of $300-330^{\circ} \mathrm{F}$ is normally recommended. $(\underline{25})$

Agitation is needed during storage to keep the particles in suspension. The LDPE is present in the final binder as small, discrete particles. It does not dissolve in the asphalt, but is supposed to act as reinforcement within the binder. The long polymer chains are also reported to adhere to the mineral aggregates, thus reducing the stripping potential. The LDPE is reported to increase the viscosity of the base asphalt by three to four times, decrease the binder temperature sensitivity and improve the penetration index. (므)

Although standard quality control tests can be used with Novophalt, the manufacturer cautions that the polyolefins can change morphology during rolling thin film oven aging. Thin film oven aging is preferred. Extraction by hot reflux with trichloroethylene is said to yield the most consistent and accurate results. (24)

Jew and Woodhams evaluated polyethylene-modified binders and mixtures in the laboratory. They reported that the addition of as little as $5 \%$ polyethylene by weight led to a tenfold increase in the apparent Brookfield viscosity. This increased viscosity necessitates longer mixing times and higher temperatures. Three-point flexural testing, conducted on binder samples at temperatures as low as $-50^{\circ} \mathrm{C}$, demonstrated increased fracture toughness. Marshall test results on mixtures with the polyethylene modified binder showed increased stability and flow values, which is an unexpected result. The addition of $8 \%$ polyethylene increased the resilient modulus of the mixture three to four times at 5 and $23^{\circ} \mathrm{C}$. Retained Marshall stability values after water immersion were also improved. (르)

Lee and Hesp investigated the low temperature fracture toughness of binders modified with stabilized PE, unstabilized PE and chlorinated PE using notched bending beam tests at 
$-20^{\circ} \mathrm{C}$. Polyethylene differs from many other polymer modifiers in that it remains a

semicrystalline solid at typical pavement service temperatures and does not dissolve in the asphalt cement. An elastomeric stabilizer is typically used to prevent the PE particles from coalescing upon cooling. They found that the binder stiffness at $-20^{\circ} \mathrm{C}$ is not greatly affected by the addition of $5-6 \% \mathrm{PE}$, but that the low temperature fracture toughness is increased. The greatest increase was demonstrated with the chlorinated PE (100\% increase). The unstabilized PE showed a 25\% increase and the stabilized PE yielded a 50\% increase. Various mechanisms to explain this toughening were presented. (29)

Little compared Novophalt mixtures at optimum (according to Marshall design parameters) and high binder contents to a binder-rich control mixture with AC-20. The evaluation of permanent deformation was based on uniaxial creep compliance, repeated-load permanent deformation and incremental static compressive loading tests. Indirect tensile creep and strength tests at temperatures down to $32^{\circ} \mathrm{C}$ and resilient modulus tests down to $4{ }^{\circ} \mathrm{C}$ were also conducted. The PE-modified binders showed increased resistance to rutting over the control mixture even at low air void contents (high binder contents). The binder-rich Novophalt mixtures were deemed "substantially superior to ... binder-rich traditional mixtures" at air void contents below 3\%. The binder-rich Novophalt mixes were more resistant to cracking than mixes at optimal binder content. Little suggests that there may be applications where binder-rich Novophalt mixes may be used to advantage to provide low permeability, improved fracture behavior and acceptable rutting resistance. A field trial of the optimal and binder-rich Novophalt mixtures at Houston Hobby Airport was reported to be performing well after two years in service. $(\underline{30})$ 


\section{A.2.5 Polyester Fibers}

Button and Epps note that fibers have been used to reinforce construction materials for over 3000 years, citing Egyptian building specifications requiring the addition of straw to the clay for brick making. (Their reference for this is Moses in the book of Exodus in the Holy Bible!) The earliest uses for fiber reinforcement of bituminous concrete pavements in the United States involved cotton fibers in the 1930's. (푸)

Typical fibers used in the more recent past for hot mix asphalt applications come in many formulations. Both natural and synthetic fibers have been used. Natural fibers include asbestos, cellulose and rock wool. Synthetic fibers include polypropylene, polyester and aramid fibers. Fibers do not react chemically with the asphalt but rather reinforce and stiffen the asphalt mastic.

() The possible advantages of using fibers to reinforce asphalt paving mixtures include reduced fatigue, thermal and reflective cracking; increased service life; and economic benefits. ( $\underline{31})$

Various fiber lengths are also available for mixes with different nominal maximum aggregate sizes. This kind of fiber acts as a reinforcement, reportedly improving binderaggregate cohesion, and reducing rutting, cracking, raveling and potholes. The fibers are added in a bag to a batch plant's mixing unit for dry mixing before the binder is added. A minimum 30second dry mixing time and 30-second wet mixing time are recommended. (ㅁ)

The Indiana Department of Transportation had extensive experience with the use of polypropylene fibers prior to this research project. Two field trials in particular were well reported. The first trial was placed as an emergency repair on a badly rutted section of interstate highway. The fiber-reinforced section exhibited significantly fewer, less severe cracks than an adjacent control section after four years in service. In addition, the fiber section performed without rutting or shoving, while the control section rutted so badly it had to be milled and replaced. The maximum rut depth in the fiber section was $9.5 \mathrm{~mm} \mathrm{(3/8} \mathrm{in.)} \mathrm{and} \mathrm{in} \mathrm{the} \mathrm{control}$ section the maximum was $63.5 \mathrm{~mm}$ (2.5 in.). The average rut depth in the fiber section was $3.2 \mathrm{~mm}(1 / 8$ in. $) .(\underline{32}, \underline{33})$

Based in part on the dramatic improvement in performance noted on this field trial, INDOT further evaluated the use of polypropylene fibers in asphalt, eventually specifying fibers for all overlays over cracked and seated pavements, a rehabilitation technique that became routine in the late 1980's and early 1990's. Research in Indiana demonstrated that the use of fibers in these overlays over cracked and seated pavement reduced the amount and severity of cracking for 
five years. After that time, the cracking increased to the same level as the section that was cracked and seated then overlaid without fibers. (프)

One reason for specifying polyester fibers for this study was to determine if they performed as well as polypropylene fibers had in the past. Polyester fibers have a higher melting point than polypropylene fibers and could, therefore, be less sensitive to high construction temperatures. (2)

Field trials in Ohio indicated that both polyester and polypropylene fibers could improve pavement performance, increase service life, reduce rutting, and reduce fatigue and thermal cracking. ( $\underline{35})$ Field trials in Delaware also demonstrated that both polyester and polypropylene fibers "offer some improvement" in reflective cracking, but the significance and cost effectiveness of those benefits were still in question at the time of their report. ( $\underline{36})$

Scherocman determined, based on laboratory data, that either polypropylene or polyester fibers added at 6 pounds per ton of mix can be used to increase the resistance to cracking and deformation. Both fiber types offered approximately the same level of improvement in the strength of the mixture. Diametral creep testing showed that the polyester fiber mix with limestone aggregate had twice the creep strength of the same mix with polypropylene fibers. $(\underline{37})$

Other research in Ohio showed that while fibers could delay the onset of reflective cracking by up to two years, they offered little improvement in rutting resistance. This was based on laboratory testing of indirect tensile strength, uniaxial creep and loaded wheel testing, as well as evaluation of field performance of six sites in Ohio. Polyester and polypropylene fibers performed similarly in that research. (효)

\section{A.2.6 Syrelf (PAC)}

Styrelf consists of a styrene butadiene block copolymer that is chemically (and irreversibly) reacted with asphalt cement to produce a homogeneous blend. Styrelf is purported to improve the low temperature susceptibility, tensile strength after elongation, elasticity and age hardening of the asphalt. Age hardening is reduced by the elimination of reactive sites on the asphalt cement that could react with oxygen and cause hardening. These properties may lead to reduced stripping, rutting, raveling, cracking and flushing on the roadway. Because the chemical co-polymerization is irreversible, Styrelf does not separate during shipping or storage. Styrelf is delivered as a ready-to-use binder. (ㅁ) (Styrelf has also been known as PAC, for Polymerized Asphalt Cement, and MAC, for Modified Asphalt Cement.) 
The manufacturer reports that Styrelf demonstrates increased tensile strength after elongation and has elastic memory to recover deformations. These properties, the manufacturer states, can lead to as much as a $750 \%$ increase in fatigue life and a $60 \%$ increase in fatigue resistance. The product can reportedly withstand ten times more loading cycles than unmodified asphalt before the onset of rutting. Conventional equipment and procedures are used for production and placement of the modified mixtures. (푸)

Khosla compared conventional AC-graded asphalts to the same asphalts modified with Styrelf. (The same asphalts were also modified by the addition of carbon black, a filler, but that comparison is not pertinent here.) Binder testing showed that Styrelf decreased the penetration (at $77^{\circ} \mathrm{F}$ ) and increased the absolute and kinematic viscosities of AC-5, AC-10 and AC-20. Mixture testing included creep fatigue and resilient modulus testing. Incremental static creep tests demonstrated that the polymer-modified mixtures developed significantly lower permanent deformation and would be expected to exhibit less rutting. Resilient modulus testing showed that the polymer mixes have lower temperature susceptibility, higher modulus at high temperatures and similar modulus at low temperatures. This indicates that the Styrelf mixtures would have improved rut resistance without affecting the cracking resistance at lower temperatures. Lastly, a diametral repeated-load indirect tensile fatigue test was used to analyze the fatigue resistance of the mixes. The polymer-modified mixes again outperformed the AC grades. The VESYS IIIA program was used to predict the performance of these mixtures if they were used in a pavement. This analysis confirmed that the Styrelf mixes would perform better than the unmodified mixes. The program estimated that a $15 \mathrm{~mm}(0.6 \mathrm{in}$.) rut would develop in four to six years in the unmodified mix section and in eight to ten years in the Styrelf section. The estimates of fatigue life varied for different regions of the country, but in all cases, the Styrelf-modified mixture had a significantly longer fatigue life than the control mixtures. (ㄴ) Similar findings have been reported in other studies of Styrelf $(\underline{41} \underline{42}, \underline{43})$.

\section{A.2.7 Styrene-Butadiene Latex (SBR)}

Styrene-Butadiene latex (SBR) is another example of Terrel and Epps' rubber category of modifiers. It is an emulsion of a random co-polymer in water. (ㅁ) The synthetic polymer is made up of a random sequence of styrene and butadiene monomers. The styrene molecules provide a strong, rigid element, while the butadiene molecules are stretchy and elastic. A combination of the two molecules, then, is both strong and elastic. $(\underline{18})$ 
SBR latex can be added to asphalt cement at the refinery or terminal, drum or batch plant, or emulsion plant. The modifier, as with any modifier, must be compatible with the asphalt so that separation does not occur or else the blend must be agitated to prevent separation. With SBR, lower storage temperatures (around $250^{\circ} \mathrm{F}$ ) prolong the effective life of the additive with minimal changes in the binder viscosity, ductility and penetration (age hardening). In addition to reduced aging, SBR is purported to increase low temperature flow, decrease high temperature flow, improve viscoelasticity and resist stripping. Depending on the base asphalt, the addition of up to 5\% SBR can reportedly improve the binder's performance grade by up to three grades. (44)

Collins et al. investigated the effects of modifying seven different asphalts with three different polymers, including SBR, SBS (styrene butadiene styrene, a thermoplastic triblock copolymer) and EVA (ethylene vinyl acetate, a thermoplastic copolymer). They studied rheological properties, examined photomicrographs showing the dispersion of the polymer in the asphalt, and related the rheological properties to rutting resistance of the mix. They stressed the importance of asphalt-polymer compatibility, noting that compatibility decreased as the AC grade (viscosity) increased. They also reported that the temperature susceptibility increased as asphalt grade increased, and the improvement in elasticity decreased as AC grade increased. These are among the reasons why many, if not most, modified asphalts start with a soft base asphalt and add the polymer to increase stiffness at high temperatures. The authors indicated this approach optimizes the performance of the binders over the entire temperature range. (ㄴ5)

Laboratory evaluations of SBR-modified binders reported in the literature indicate mixed performance. For example, Button and Little concluded that SBR-modified AC-5 would be more resistant to rutting based on its higher creep compliance. (느) Lee and Demirel, however, determined that the addition of SBR had no effect on rutting based on static uniaxial compressive creep tests of modified mixtures. Lee and Demirel also found that the addition of SBR had mixed effects on stripping resistance depending on the aggregate and binder used, indicating the importance of compatibility of the binder and aggregate. (구)

SBR did prove successful in reducing reflective cracking in an overlay over concrete, though not as effective as sawing and sealing joints, in a study by King and Doucet. They compared the construction and field performance of waterproofing membranes to sawing and sealing joints in the overlay and using SBR latex-modified asphalt in the overlay. The SBR was added to an AC-10 and was also compared to a conventional AC-30 mixture. No production or construction difficulties were noted with the latex-modified overlay. After three years, 
performance of the SBR sections was superior to the unmodified overlay and the membranes. No rutting was noted in either the control or modified mixes. (뇨)

A 1996 survey of State Asphalt Pavement Association Executives members showed mixed field results with SBR, as well as SBS and SB. Louisiana was reported to require all surface and intermediate courses to be modified with either SBS or SBR. Virginia reportedly required SBS or SBR for mixes used in special applications. Alabama reported that they had used SBR on approximately $20 \%$ of their centerline mileage since the mid-1980's. No significant increase in life was attributed to the polymer, though they were hoping to see improved rutting resistance. Colorado had used SBR since about 1975 sporadically. Several other states reported experimental usage, but the predominant message was that performance improvements had, in most cases, not been verified. For example, Pennsylvania first used SBR on an experimental project; over ten years later, SBR was still considered experimental because it performed similarly to the unmodified control section. (노)

\section{$\underline{\text { A.4 Test Methods }}$}

A variety of test methods have been used to evaluate modified binders over the years. Conventional tests were initially used, then specialized tests were added to evaluate the unique features of modified binders. With the advent of Superpave performance grading, modified binder testing became the focus of intensive research that continues to this day. The following summarizes some of the test methods that have been used and their success at characterizing modified binders.

\section{A.4.1 Binder Tests}

Early work with modified binders used conventional binder tests to characterize the materials. Berkheimer used ring and ball softening point, penetration, ductility, torsional recovery, toughness-tenacity, viscosity and other tests (17).

Lee and Hesp (29) and Hui et al. ( $\underline{8})$ used low-temperature fracture to evaluate the low temperature cracking properties of modified binders. This test uses a notched beam subjected to third-point bending according to ASTM E399-90. 
Superpave introduced some new test methods into the asphalt industry. Anderson and Kennedy outlined the development of the Superpave Performance Grading specifications in 1993. These specifications were supposed to be equally applicable to modified and unmodified binders. In fact, the term "asphalt binder" was coined to include both modified and unmodified asphalt cements. The new specifications retain some old tests, such as Cleveland open cup flash point determination for safety and the mass loss determination to limit the use of flux oils and volatile materials that are detrimental to the binder quality over time. The new tests are performance based and include:

- The inverse of the loss compliance $\left(\mathrm{G}^{*} / \sin \delta\right)$, determined in the dynamic shear rheometer (DSR), which relates to rutting at high temperatures.

- The dissipated energy $\left(\mathrm{G}^{*} \sin \delta\right)$, also measured in the DSR, which is a surrogate for fatigue at intermediate temperatures.

- The stiffness $(\mathrm{S}(\mathrm{t}))$ and slope of stiffness versus time curve $(\mathrm{m})$ measured in the bending beam rheometer (BBR), which relate to thermal cracking.

- The strain at failure, measured in direct tension, which was an optional test also related to thermal cracking.

Anderson and Kennedy stressed that the binder must be aged to simulate age hardening during mixing/laydown and during service, and the binder must be tested at temperatures appropriate for the distress type being evaluated and the climate where the binder will be used. (50)

With the implementation of the Superpave Performance Grading tests and specification, many researchers have attempted to use the new procedures "as is" to evaluate modified binders. $(\underline{45}, \underline{52}-54)$

During the development of the PG tests and specifications, King et al. used several different test methods to look at one base asphalt, vacuum distilled to four different penetration grades then modified with three levels of polymer addition. They compared conventional high temperature binder tests (ring and ball softening point, kinematic and absolute viscosities, and penetration) to mixture rutting tests, including the Hamburg wheel tracking device and French LCPC rut tester. They found that the conventional tests did not adequately predict the wheel tracking results for polymer modified binders. King also evaluated the binders in the dynamic shear rheometer, using several different parameters that were being considered for the PG specifications. Both the viscous modulus, G", and the complex shear modulus, G*, were able to predict rut depths reasonably well in both the LCPC and Hamburg devices. They stressed that the test temperatures and the frequency of oscillation need to be selected appropriately. When 
comparing results of the binder tests to the torture tests, the same temperature should be used for both. The test temperature should also replicate the most extreme temperatures that will exist in the actual pavement. (ㅌ1)

In a similar paper the following year, King et al. looked at the conventional and proposed Superpave tests for low temperature cracking. They found that low temperature penetration $\left(4^{\circ} \mathrm{C}\right)$, low temperature ductility $\left(4^{\circ} \mathrm{C}\right)$ and Fraas brittle point were not able to predict the cracking temperature for unmodified binders and were even less reliable for modified binders. The bending beam rheometer, however, did relate to the temperature at which cracking occurred. The tests also showed that reacted SB polymers significantly reduced the low temperature stiffness of an asphalt and that elastomers could withstand greater strains before fracturing than unmodified binder of the same stiffness. (므)

Tayebali et al. tested crumb rubber modified binders in the dynamic shear rheometer and concluded that the test method was applicable to the modified binders they investigated. They added 40 and 80 mesh rubber to AC-20 and AC-10 asphalts at contents up to $14 \%$. They also concluded that the particle size of the rubber did not change the binder grade of the modified asphalt. Their findings indicated that $7 \%$ rubber increased the high temperature grade by one 6degree increment and that $14 \%$ rubber increased the high temperature grade two increments. $(\underline{53})$

Dongré et al. also used the original Superpave binder specifications to characterize a number of modified binders, including Styrelf, Ultrapave (SBR) and Novophalt, among others. They compared the test results to observed field performance at two test sites in Texas. Their findings indicated that the high temperature rutting parameter was accurate, but that the intermediate temperature fatigue parameter was not. They also concluded that the ability to predict low temperature cracking was improved when direct tension tests supplement the bending beam rheometer results. $(\underline{54})$

As Bahia et al. pointed out, however, the original Superpave binder tests were developed for conventional, unmodified asphalt binders, and some of the underlying assumptions do not apply to modified systems. They recommended that binders be identified as "simple" or "complex." Simple binders would conform to the assumptions of the Superpave binder protocols and could be tested using those methods. Complex binders, on the other hand, would violate these assumptions and would need revised or new tests to characterize their behavior. The assumptions identified by Bahia et al. include the following:

- Binders are linear viscoelastic materials with no strain/stress dependency.

- Binders are Newtonian over a wide range of shear rates. 
- Binders are homogeneous and isotropic, which poses a problem for particulate additives.

- Binders have similar time-temperature equivalencies.

- Binders are not thixotropic.

- Oxidation is the main mechanism affecting stability of the binders. (ㄷ5)

There are modified systems, and even some unmodified asphalts, that violate these assumptions. As a result, Bahia et al. recommended determining if a binder is rheologically simple based on considerations of strain dependency, thixotropy, and type and amount of modifier. They also recommended actually measuring the time-temperature equivalency of a binder by conducting a temperature sweep rather than assuming all binders have the same equivalency. They suggested the need for new tests and modifications to the existing protocols for low temperature properties, thermal stability and aging behavior. (ㄷ5)

Under Bahia's proposed testing scheme, a binder would be subjected to time and strain sweeps in the dynamic shear rheometer (DSR) at high and intermediate temperatures to determine if it is simple or complex. A change of more than $10 \%$ over the sweep ranges would signify that the binder is complex. Simple binders would be evaluated to detect the presence of particulates using a Particulate Additive Test (PAT). Then a Laboratory Asphalt Stability Test (LAST) would be used to measure thermal degradation and separation of the binder. (ㄷ5)

In the final analysis, however, classifying binders as simple or complex "was found to be unnecessary." Bahia et al. recommended, based on their extensive work under NCHRP 9-10, that all binders be screened for the presence of particulates and for storage stability. Improved test protocols and parameters were offered for the characterization of rutting, fatigue and thermal cracking behavior. (ㅌ6)

The Particulate Additive Test (PAT) uses n-octane and toluene to essentially wash away the asphalt binder, leaving the particulate additives behind on a fine screen $(75 \mu \mathrm{m})$. The $\mathrm{n}$ octane removes the asphalt leaving behind all additives. Some highly polar fractions of the asphalt may adhere to the additives as well. The results indicate the presence of an additive in the binder. The toluene is then used to determine the nature of the additive. Toluene will dissolve additives that are soluble in asphalt, leaving the insoluble additives. (드)

The Laboratory Asphalt Stability Test (LAST) is used to evaluate the potential for separation or thermal degradation of a binder under hot storage conditions similar to those encountered during hot mix production. The test basically consists of a laboratory-scale vertical 
storage tank with agitation and internal heating. A sample of binder is evaluated before and after storage using the dynamic shear rheometer. Samples are also taken and tested from the top and bottom of the tank using a pipette. Comparison of the results before and after storage indicates the potential for thermal degradation, and comparison of top and bottom samples indicates the tendency to separate. (다) AASHTO protocols have been developed for both of these test methods.

Bahia et al. also recommended changes in the Performance Graded binder specifications and test protocols. They indicated that the current specification parameters are inadequate to evaluate the rheological and damage behavior of mixtures. Instead, they suggested using the same testing equipment in different ways to more fully assess binder damage. Their proposed changes included:

- Adding periodic screening tests for quality control including the PAT, LAST, glass transition temperature and physical hardening test.

- Replacing the rutting parameter $\mathrm{G}^{*} / \sin \delta$ with $\mathrm{G}_{v}$, the viscous creep stiffness, measured in a repeated creep-loading test in the DSR.

- Replacing the fatigue parameter $\mathrm{G}^{*} \sin \delta$ with $\mathrm{N}_{\mathrm{p}}$, determined based on changes in $\mathrm{G}^{*}$ and $\delta$ during a repeated controlled stress test in the DSR. $\mathrm{N}_{\mathrm{p}}$ is an estimate of the number of loading cycles to crack propagation.

- Determining critical thermal cracking temperatures based on thermal stress and strain at failure rather than on $\mathrm{S}$ and $\mathrm{m}$.

- Using a binder's low shear viscosity, as measured in the rotational viscometer, to estimate mixing and compaction temperatures. (ㅎ6)

Proposed AASHTO specifications and test methods have been prepared detailing these changes.

Bahia et al. described the relationship between binder and mixture properties in a 2001 AAPT paper. In this work, they related the proposed new binder parameters for rutting and fatigue, $G_{v}$ and $N_{p}$ respectively, to mixture behavior. The mixture rutting potential was evaluated using the Repeated Shear at Constant Height test and fatigue behavior was analyzed using flexural beam fatigue. Results showed that the proposed new parameters offer improved measures of the binder properties that relate to mixture rutting and fatigue cracking. (57) 


\section{A.4.2 Mixture Tests}

Hanson et al. characterized rubber-modified hot mix behavior using conventional tests circa 1993. That is, they employed resilient modulus, gyratory densification properties in the Corps of Engineers gyratory, indirect tensile strength, tensile strength ratio and confined creep. Similar tests were run on field cores and laboratory-prepared specimens. Field mixtures were also evaluated in terms of density, air voids and rut depth. (11)

The Kentucky Transportation Cabinet designed a field trial in 1996 to compare the performance of five modified binders all meeting a PG70-22 grade and a control PG64-22. They wanted to know if different methods of modification resulted in the same field performance. The modified binders evaluated included two brands of SBS, one SBR, one chemically modified (gelled) asphalt and an unmodified asphalt. The binders were all used in the same Marshall mix design, which met most Superpave requirements, but did pass through the lower portion of the restricted zone. This mixture had exhibited good performance in the past. (타)

The mixture performance was evaluated in the laboratory using the Superpave Shear Tester (Frequency Sweep, Repeated Shear at Constant Stress Ratio and Repeated Shear at Constant Height), loaded wheel testing (Hamburg Wheel Tracking, Asphalt Pavement Analyzer (APA) and Georgia Loaded Wheel Tester) and tensile strength ratio (TSR) testing. The results showed that the gelled and SBS modified mixtures would show less rutting than the other mixtures; the control mixture would be expected to exhibit the most rutting. The Hamburg and APA test results compared with the TSR results gave conflicting results for the stripping potential of the mixtures, but the authors felt the wheel tracking tests were useful tools for evaluating both rutting and stripping. The ultimate conclusion was that the different binders will not yield the same field performance. $(\underline{58})$

Discussion after the paper pointed to some potential flaws in the study. Although all binders, except the control, technically met the requirements for a PG70-22, some also met grades as high as PG76 and even PG82. It was suggested that comparing the performance of a PG70 to a PG82 in terms of rutting is not a valid comparison; the PG82 would be expected to perform better. The reliance of the study on wheel tracking tests was also criticized. Other evaluations suggest that wheel trackers do not provide reliable indicators of rutting or stripping resistance. $(\underline{58})$

King et al. also used the Hamburg wheel tracking device and the French LCPC rut tester to evaluate mixes modified with chemically reacted styrene-butadiene block copolymer. The 
Hamburg device has been used in Germany since the mid-1970's. Samples of the mixture being evaluated are submerged in a heated water bath, simulating both rutting and stripping behavior. A steel wheel tracks back and forth over the test slab. The sinusoidal loading produces about a 0.1 second loading period followed by a 0.9 second rest. The tests are typically conducted at 40 and $50^{\circ} \mathrm{C}\left(104-122^{\circ} \mathrm{F}\right)$ for 20,000 cycles or until a $20 \mathrm{~mm}(0.8 \mathrm{in}$.) rut develops. The resulting deformation curve can be analyzed to determine the post-compaction densification, creep deformation, onset of stripping or shear failure, if present, and subsequent rapid deformation of the damaged specimen. (ㅌ1)

In 1997, Stuart and Mogawer attempted to provide partial validation of the Superpave binder specifications by comparing the performance of mixtures with five different modifiers based on the full-scale Accelerated Loading Facility (ALF), laboratory-scale wheel tracking devices (French LCPC Pavement Rutting Tester, Georgia Loaded Wheel Tester and Hamburg Loaded Wheel Tester) and the Superpave shear tester. The mixtures were the same except for the binder used. In general, they found that as the binder high temperature stiffness $\left(\mathrm{G}^{*} / \sin \delta\right)$ increased, the mixture rutting resistance also increased, as expected. The exception to this was that the Novophalt modified binder had a lower stiffness (meeting a PG76 grade) than the Styrelf binder (PG82), but showed less rutting under the ALF. One possible explanation for this, offered during the discussion by King, is that the polyethylene can separate and form "microcrystalline aliphatic domains over time," thus exhibiting higher binder stiffness than when initially measured in the DSR. (This is another example of the time-dependent nature of some modifiers.) (무)

Stuart and Mogawer concluded that the wheel tracking tests were useful for differentiating between widely different binder grades, but were not as successful in differentiating between relatively similar grades, such as PG64-22 and PG70-22. No single lab test proved to be clearly better at simulating field performance under the ALF. (모)

Aschenbrener reported on an evaluation of the Hamburg wheel-tracking device for use in predicting the susceptibility of a mixture to moisture damage. He described the creep slope, stripping slope and stripping inflection point and their significance. (These are discussed further in section 5.2.1 of this report.) Aschenbrener then went on to summarize the results of testing materials from seven pavements of known good performance with respect to moisture damage, five pavements that were still in service but required extensive maintenance (termed "high maintenance pavements") and eight pavements that failed within one year. The results of the wheel-tracking tests showed excellent correlation to field performance. In particular, the stripping inflection point related well to field stripping performance. The device was found to be 
sensitive to binder stiffness, aggregate quality, source or type of binder, the presence of antistripping additives, short-term aging and compaction temperature. (느)

\section{A.4.3 Field Performance Properties}

Brown et al. used field performance and a literature review to assess the effectiveness of SBR in HMA. The field performance was evaluated based on manual condition surveys that measured rutting, transverse cracking, raveling and bleeding. They also used the Alabama Highway Department's pavement management system to obtain mean rut depth (rut depth/square root of ESAL), present serviceability index, friction number, condition rating and transverse cracking. (61) These are all relatively standard performance measures appropriate for any field evaluation of asphalt pavements.

\section{A.5 Performance of Modified Asphalts}

The Indiana Department of Transportation had some experience with various modified asphalts prior to this research project, though it was not extensive. Previous research projects had dealt with the field evaluation of PAC 30 (Styrelf), fibers and crumb rubber (dry process).

In May 1986, INDOT placed PAC 30 mixtures in a test section on US 41 through Terre Haute, Indiana. This roadway carried about 38,000 vehicles per day, with approximately $10 \%$ trucks, at the time of the evaluation. The mixtures were placed according to the 1985 standard specifications, which used a "cookbook" approach rather than a Marshall mix design. A 1990 report documents the performance of the test section and an unmodified control section. The modified sections showed reduced rutting (up to $16 \mathrm{~mm}$ (5/8 in.)) compared to the control sections (up to $28.6 \mathrm{~mm}$ (1-1/8in.)), but there was still a significant amount of rutting. Based in part on these results, the PAC binder (Styrelf) used on the I-465 field trial used an AC-20 asphalt as the base as opposed to the AC-10 used on US 41. (2)

A field trial of neoprene latex on SR67 near Camby, Indiana, showed comparable rutting in the control and test sections in 1990. Rut depths of 3.2 to $7.9 \mathrm{~mm}$ (1/8 to $5 / 16$ in.) were found in both sections. Reflective cracking appeared to be comparable as well. This test section was placed in 1988. (주) 
Brown et al. investigated the performance of six existing SBR-modified pavements in Alabama and compared their performance to the performance of similar control sections. The pavement sections ranged in age from two to five years. At the time of the condition surveys, the use of SBR did not significantly improve the field performance of the test sections, though this data was considered preliminary and further analysis was deemed necessary. (ㅁ1) 